\title{
Republic of Belarus: Statistical Appendix
}

This Statistical Appendix for the Republic of Belarus was prepared by a staff team of the International Monetary Fund as background documentation for the periodic consultation with the member country. It is based on the information available at the time it was completed on April 22, 2004. The views expressed in this document are those of the staff team and do not necessarily reflect the views of the government of the Republic of Belarus or the Executive Board of the IMF.

The policy of publication of staff reports and other documents by the IMF allows for the deletion of market-sensitive information.

To assist the IMF in evaluating the publication policy, reader comments are invited and may be sent by e-mail to publicationpolicy@imf.org.

Copies of this report are available to the public from

International Monetary Fund • Publication Services

$70019^{\text {th }}$ Street, N.W. • Washington, D.C. 20431

Telephone: (202) 623-7430 • Telefax: (202) 623-7201

E-mail: publications@imf.org •Internet: http://www.imf.org

Price: $\$ 15.00$ a copy

\section{International Monetary Fund \\ Washington, D.C.}





\title{
INTERNATIONAL MONETARY FUND
}

\author{
REPUBLIC OF BELARUS
}

\section{Statistical Appendix}

Prepared by Veronica Bacalu, Etibar Jafarov, Thomas Richardson, and Jérôme Vacher

Approved by the European Department

April 22, 2004

Contents

Page

Basic Data 3

Statistical Appendix Tables

1. Gross Domestic Product by Sector, 1999-2003 ……………...................................

2. Gross Domestic Product by Expenditure, 1999-2003 .................................................

3. Growth of Gross Domestic Product by Expenditure, 1999-2003 …………................6

4. Growth of Gross Domestic Product by Sector, 1999-2003 ………………….............

5. Capital Investment by Sector, 1999-2003 ……..........................................................

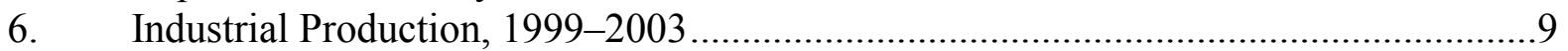

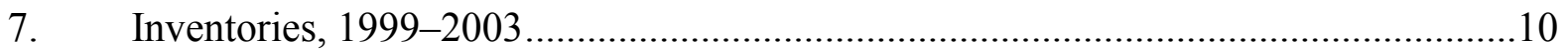

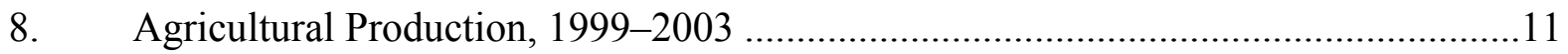

9. Production and Consumption of Energy, 1999-2003 ……………...........................12

10. Change in Consumer and Producer Prices, 2000-03 ................................................13

11. Administered Prices of Household Services, 2000-03 ..............................................14

12. Tariffs and Cost Coverage of Utility Prices for Households, 1999-2003 ....................15

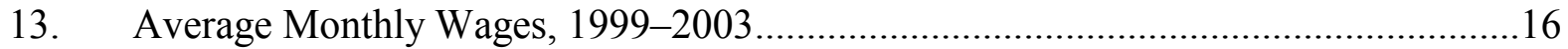

14. Price and Wage Developments, 1999-2003 ……...................................................17

15. Money Income and Expenditures of the Population, 1999-2003 …...........................18

16. Labor Market Indicators, 1999-2003 ………….................................................19

17. Average Monthly Employment by Sector, 1999-2003 ……………………..............2

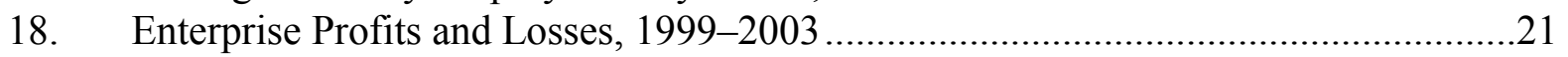

19. Interenterprise Arrears and the Barter Economy, 1999-2003 …………...................22

20. Sectoral Distribution of Energy Debts of Enterprises, 1999-2003 …........................23

21. General Government Operations, 1999-2003 (In rubels) .........................................24

22. General Government Operations, 1999-2003 (In percent of GDP)............................25

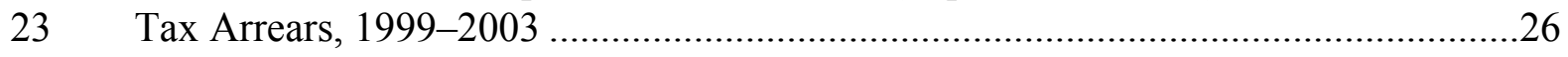

24. General Government Debt, 1999-2003 ……………………………………........27 


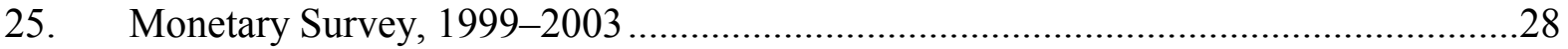

26. Accounts of the National Bank of Belarus, 1999-2003 ……………………............29

27. Deposit Money Banks' Accounts, 1999-2003 …………………...............................30

28. National Bank's Directed Credits, 1999-2003 ……...................................................31

29. Composition of Bank Lending by Type of Credit and Sector, 1999-2003 …...............32

30. Auction of Securities Issued by the Ministry of Finance, 2001-03 ………..................33

31. Minimum Reserve Requirements, 1999-2003 ........................................................

32. Interest Rates of the National Bank of Belarus, 1999-2003 .......................................35

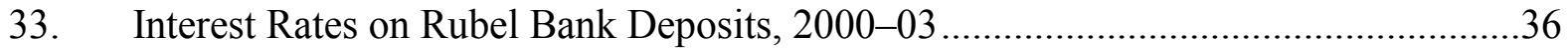

34. Interest Rates on Rubel Bank Credit, 2000-03 .......................................................

35. Interest Rates on New Foreign Exchange Credits and Deposits, 2000-03 ...................38

36. Structural Characteristics of the Banking Sector, 1999-2003 ….................................39

37. Selected Banking Indicators, 1999-2003 …………................................................40

38. Six Largest Commercial Banks, Selected Indicators, 1999-2003 .............................41

39. Commercial Banks and Branches of Foreign Banks.................................................42

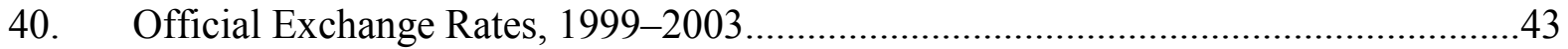

41. Direction of Trade: Exports and Imports, 1999-2003 …………………...................4

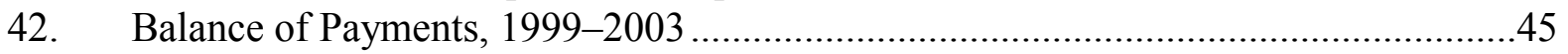

43. Selected International Liabilities, 1999-2003 ...........................................................46

44. Disbursements and Payments on Medium- and Long-Term Public and Publicly-Guaranteed Debt, 1999-2003 ………………........................................47

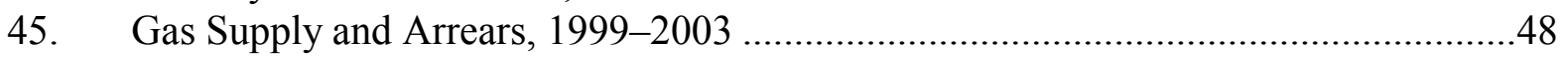

46. Import and Consumption of Natural Gas, Monthly, 2000-03 ..................................49

47. Production, Trade and Consumption of Crude Oil, Monthly, 2000-03 ………..........50

48. Production, Trade and Consumption of Electricity, Monthly, 2000-03 ......................51

49. Exports and Imports of Oil Products, Monthly, 2000-03 .........................................52

50. Transformation of Property, 1999-2003 ………………............................................53 
Belarus: Basic Data, 1999-2003

\begin{tabular}{|c|c|c|c|c|c|}
\hline & 1999 & 2000 & 2001 & 2002 & 2003 \\
\hline \multicolumn{6}{|l|}{ Social and demographic indicators } \\
\hline Area $\left(\mathrm{km}^{2}\right)$ & 207,600 & 207,600 & 207,600 & 207,600 & 207,600 \\
\hline Population (in thousands) & 10,020 & 9,990 & 9,951 & 9,899 & 9,849 \\
\hline Urban & 6,985 & 7,014 & 7,031 & 7,037 & 7,046 \\
\hline Rural & 3,034 & 2,977 & 2,920 & 2,862 & 2,803 \\
\hline Population density (inhabitants per sq. km.) & 48 & 48 & 48 & 48 & 47 \\
\hline Life expectancy at birth (in years) & 67.9 & 69.0 & 68.5 & 68.0 & \\
\hline Infant mortality rate (per thousand) & 11.5 & 9.3 & 9.1 & 7.8 & 7.7 \\
\hline Annual population growth rate (in percent) & -0.3 & -0.3 & -0.4 & -0.5 & -0.5 \\
\hline GDP (in millions of U.S. dollars) $1 /$ & 12,099 & 12,728 & 12,329 & 14,651 & 17,513 \\
\hline GDP per capita (in U.S. dollars) $1 /$ & 1,204 & 1,270 & 1,239 & 1,477 & 1,765 \\
\hline & \multicolumn{5}{|c|}{ (Percentage changes in constant prices) } \\
\hline \multicolumn{6}{|l|}{ Output } \\
\hline GDP & 3.4 & 5.8 & 4.7 & 5.0 & 6.8 \\
\hline \multicolumn{6}{|l|}{ Of which: } \\
\hline Industry & 8.8 & 8.6 & 7.2 & 6.1 & 9.1 \\
\hline Agriculture & -7.0 & 8.8 & 1.6 & 3.1 & 7.8 \\
\hline Industrial production & 10.3 & 7.8 & 5.9 & 4.5 & 6.8 \\
\hline & \multicolumn{5}{|c|}{ (Percentage changes) } \\
\hline \multicolumn{6}{|l|}{ Prices and Wages } \\
\hline Consumer prices & 293.7 & 168.6 & 61.1 & 42.6 & 28.4 \\
\hline Consumer prices (end-of-period) & 251.2 & 107.5 & 46.1 & 34.8 & 25.4 \\
\hline Producer prices (end-of-period) & 245.1 & 168.0 & 39.1 & 42.7 & 28.6 \\
\hline Average wage & 322.4 & 198.0 & 108.8 & 53.8 & 34.0 \\
\hline & \multicolumn{5}{|c|}{ (In U.S. dollars) } \\
\hline Average wage (end-of-period) 1/ & 79 & 75 & 106 & 115 & 139 \\
\hline Minimum wage (end-of-period) $1 /$ & 3 & 3 & 6 & 11 & 22 \\
\hline & \multicolumn{5}{|c|}{ (In percent of GDP) } \\
\hline \multicolumn{6}{|l|}{ General government finance 2/ } \\
\hline Revenue & 45.3 & 45.7 & 44.9 & 42.8 & 44.7 \\
\hline Expenditure 3/ & 47.3 & 45.9 & 46.8 & 44.7 & 45.9 \\
\hline Balance 3/ & -2.0 & -0.2 & -1.9 & -1.9 & -1.2 \\
\hline & \multicolumn{5}{|c|}{ (Percentage changes) } \\
\hline \multicolumn{6}{|l|}{ Money and credit } \\
\hline Rubel broad money & 195 & 124 & 97 & 60 & 71 \\
\hline Banking system net domestic credit 4/ & 143 & 188 & 66 & 54 & 75 \\
\hline Refinance rate (percent per annum, end-of-period) & 120 & 85 & 48 & 38 & 28 \\
\hline & \multicolumn{5}{|c|}{ (In millions of U.S. dollars; unless otherwise indicated) } \\
\hline \multicolumn{6}{|l|}{ Merchandise trade } \\
\hline Exports of goods & 5,646 & 6,641 & 7,334 & 7,965 & 10,010 \\
\hline Imports of goods & $-6,126$ & $-7,525$ & $-8,141$ & $-8,879$ & $-11,085$ \\
\hline Trade balance & -480 & -884 & -807 & -914 & $-1,075$ \\
\hline Current account balance (in percent of GDP) & -1.6 & -2.5 & -3.5 & -2.6 & -2.6 \\
\hline Exchange rate (in rubels per U.S. dollar; end-of-period) 5 , & 320 & 1,180 & 1,580 & 1,920 & 2,156 \\
\hline (in rubels per U.S. dollar; period average) & 277 & 800 & 1,383 & 1,784 & 2,052 \\
\hline Gross convertible official reserves $6 /$ & 309 & 357 & 359 & 457 & 474 \\
\hline
\end{tabular}

Sources: Belarusian authorities; and IMF staff estimates.

1/ Measured at the official exchange rate. Since 2001, IMF staff estimate.

2/ Consolidated (cash) position, including local governments, budgetary funds, and the social protection fund.

3/ Includes an adjustment for discrepancy between monetary and fiscal data.

4/ Unadjusted for exchange rate variation.

5/ Data have been revised backward to reflect the redenomination of the rubel on January 1, 2000,

which removed three zeros from the currency.

6/ Based on NBB balance sheet data. 
Table 1. Belarus: Gross Domestic Product by Sector, 1999-2003 1/

(At current prices, unless otherwise indicated)

\begin{tabular}{lrrrrr}
\hline & 1999 & 2000 & 2001 & 2002 & 2003 \\
\hline GDP at market prices & 3,026 & 9,134 & 17,173 & 26,138 & 35,930 \\
GDP at factor cost & 2,625 & 7,807 & 14,969 & 22,483 & 30,522 \\
Industry & 836 & 2,421 & 4,474 & 6,647 & 9,180 \\
Agriculture and forestry & 384 & 1,105 & 1,778 & 2,647 & 2,985 \\
Construction & 176 & 588 & 1,007 & 1,528 & 2,151 \\
Transportion/communications & 346 & 1,012 & 1,975 & 2,710 & 3,735 \\
Trade and catering & 290 & 869 & 1,724 & 2,600 & 3,560 \\
Material supply and procurement & 48 & 122 & 189 & 226 & 287 \\
Housing and public utilities & 128 & 387 & 954 & 1,368 & 1,998 \\
Health care & 94 & 283 & 619 & 995 & 1,613 \\
Education, culture, and science & 166 & 512 & 1,086 & 1,698 & 2,151 \\
Other & 157 & 509 & 1,164 & 2,064 & 2,862 \\
& & & & & \\
Industry & $($ In & percent of nominal GDP at factor cost $)$ & \\
Agriculture and forestry & 31.9 & 31.0 & 29.9 & 29.6 & 30.1 \\
Construction & 14.6 & 14.2 & 11.9 & 11.8 & 9.8 \\
Transportion/communications & 6.7 & 7.5 & 6.7 & 6.8 & 7.0 \\
Trade and catering & 13.2 & 13.0 & 13.2 & 12.1 & 12.2 \\
Material supply and procurement & 11.0 & 11.1 & 11.5 & 11.6 & 11.7 \\
Housing and public utilities & 1.8 & 1.6 & 1.3 & 1.0 & 0.9 \\
Health care & 4.9 & 4.9 & 6.4 & 6.1 & 6.5 \\
Education, culture and science & 3.6 & 3.6 & 4.1 & 4.4 & 5.3 \\
Other & 6.3 & 6.6 & 7.3 & 7.6 & 7.0 \\
Total & 6.0 & 6.5 & 7.7 & 9.2 & 9.5 \\
\hline & 100.0 & 100.0 & 100.0 & 100.0 & 100.0 \\
\hline
\end{tabular}

Source: Ministry of Statistics and Analysis; and Fund staff estimates.

1/ Data have been revised backward to reflect the redenomination of the rubel on January 1, 2000 which removed three zeros from the currency. 
Table 2. Belarus: Gross Domestic Product by Expenditure, 1999-2003 1/

(At current prices, unless otherwise indicated)

\begin{tabular}{|c|c|c|c|c|c|c|c|}
\hline & \multirow[t]{2}{*}{1999} & \multirow[t]{2}{*}{2000} & \multirow[t]{2}{*}{2001} & \multirow[t]{2}{*}{2002} & \multicolumn{3}{|c|}{2003} \\
\hline & & & & & Q1 & Q2 & Q3 \\
\hline & \multicolumn{7}{|c|}{ (In millions of rubels) } \\
\hline GDP at market prices & 3,026 & 9,134 & 17,173 & 26,138 & 6,910 & 8,466 & 10,313 \\
\hline Consumption of goods and services & 2,365 & 6,977 & 13,597 & 21,047 & 5,817 & 6,727 & 8,139 \\
\hline Household consumption & 1,715 & 5,012 & 9,531 & 15,114 & 4,142 & 4,694 & 6,121 \\
\hline Public consumption & 650 & 1,965 & 4,066 & 5,933 & 1,675 & 2,033 & 2,018 \\
\hline General government & 590 & 1,779 & 3,701 & 5,497 & 1,545 & 1,893 & 1,880 \\
\hline Public organizations & 60 & 186 & 365 & 436 & 130 & 140 & 138 \\
\hline Gross capital formation & 717 & 2,320 & 4,081 & 5,797 & 1,053 & 2,037 & 2,688 \\
\hline Gross fixed capital formation 2/ & 797 & 2,302 & 3,893 & 5,747 & 1,030 & 1,937 & 2,445 \\
\hline Changes in inventories & -79 & 18 & 188 & 50 & 23 & 100 & 243 \\
\hline Trade balance $3 /$ & -73 & -291 & -610 & -980 & -71 & -256 & -443 \\
\hline \multirow[t]{2}{*}{ Statistical discrepancy } & 17 & 128 & 105 & 274 & 111 & -42 & -71 \\
\hline & \multicolumn{7}{|c|}{ (In percent of nominal GDP at market prices) } \\
\hline Consumption of goods and services & 78.1 & 76.4 & 79.2 & 80.5 & 84.2 & 79.4 & 78.9 \\
\hline Household consumption & 56.6 & 54.9 & 55.5 & 57.8 & 59.9 & 55.4 & 59.3 \\
\hline Public consumption & 21.5 & 21.5 & 23.7 & 22.7 & 24.3 & 24 & 19.6 \\
\hline General government & 19.5 & 19.5 & 21.6 & 21.0 & 22.4 & 22.3 & 18.3 \\
\hline Public organizations & 2.0 & 2.0 & 2.1 & 1.7 & 1.9 & 1.7 & 1.3 \\
\hline Gross capital formation & 23.7 & 25.4 & 23.8 & 22.2 & 15.2 & 24.1 & 26.1 \\
\hline Gross fixed capital formation 2/ & 26.3 & 25.2 & 22.7 & 22.0 & 14.9 & 22.9 & 23.7 \\
\hline Changes in inventories & -2.6 & 0.2 & 1.1 & 0.2 & 0.3 & 1.2 & 2.4 \\
\hline Trade balance $3 /$ & -2.4 & -3.2 & -3.6 & -3.7 & -1 & -3 & -4.3 \\
\hline Statistical discrepancy & 0.6 & 1.4 & 0.6 & 1.0 & 1.6 & -0.5 & -0.7 \\
\hline
\end{tabular}

Source: Ministry of Statistics and Analysis.

1/ Data have been revised backward to reflect the redenomination of the rubel on January 1, 2000 which removed three zeros from the currency.

2/ Includes residential investment.

3/ Provisional data, not fully consistent with recently revised balance of payments data (Table 43). 


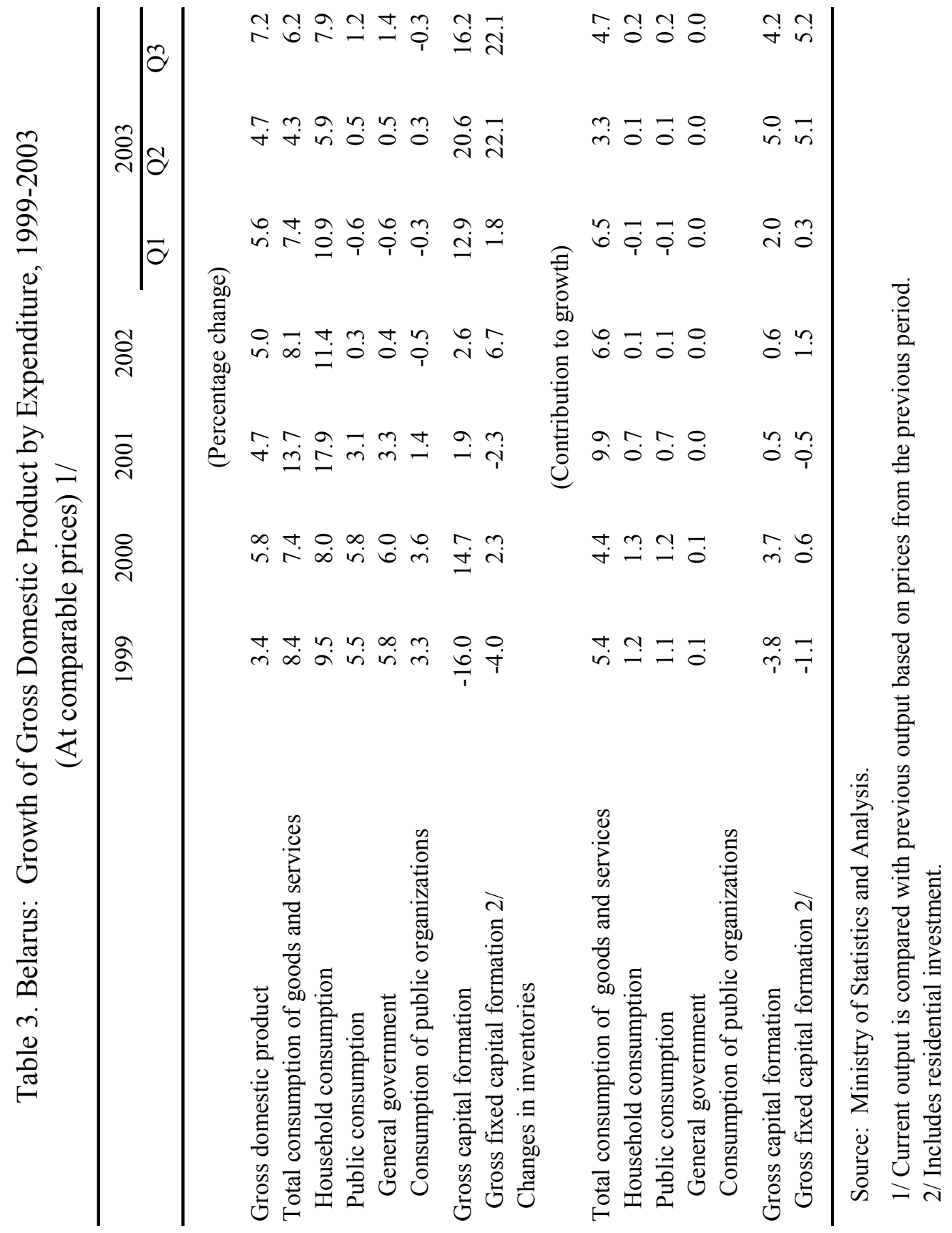


Table 4. Belarus: Growth of Gross Domestic Product by Sector, 1999-2003 (At comparable prices) 1/

\begin{tabular}{|c|c|c|c|c|c|c|c|c|}
\hline & \multirow[t]{2}{*}{1999} & \multirow[t]{2}{*}{2000} & \multirow[t]{2}{*}{2001} & \multirow[t]{2}{*}{2002} & \multicolumn{4}{|c|}{2003} \\
\hline & & & & & Q1 & Q2 & Q3 & Q4 \\
\hline & & & \multicolumn{3}{|c|}{ (Percentage chang } & \multicolumn{2}{|c|}{11.4} & \\
\hline GDP at market prices & 3.4 & 5.8 & 4.7 & 5.0 & 5.6 & 4.7 & 7.2 & 8.8 \\
\hline Industry & 8.8 & 8.6 & 7.2 & 6.1 & 8.9 & 7.7 & 9.3 & 10.2 \\
\hline Agriculture & -7.0 & 8.8 & 1.6 & 3.1 & -0.3 & -2.6 & 6.6 & 22.9 \\
\hline Forestry & -17.9 & 19.8 & -3.2 & 1.0 & -12.6 & -5.2 & 40.9 & -13.8 \\
\hline Construction & -2.4 & -2.1 & -5.2 & 10.8 & 4.4 & 9.6 & 17.1 & 12.1 \\
\hline Material supply & 0.4 & -3.4 & -5.9 & -15.1 & 7.2 & 8.2 & 2.4 & -8.2 \\
\hline Transport & 1.5 & 1.9 & 1.8 & 2.2 & 5.1 & 4.8 & 10.5 & 8.7 \\
\hline Communications & 6.3 & 3.8 & 5.1 & 3.8 & 13.3 & 6.7 & 6.7 & 9.0 \\
\hline Trade and catering & 8.2 & 9.6 & 19.5 & 8.4 & 8.1 & 4.6 & 7.0 & 18.1 \\
\hline Procurement & -11.4 & 3.8 & -26.5 & 11.7 & 4.4 & -5.3 & -34.5 & 44.5 \\
\hline Housing & 1.1 & 1.1 & 2.0 & 1.0 & 1.1 & 0.8 & 0.4 & 0.5 \\
\hline Public utilities & 0.9 & 0.3 & 3.5 & -2.8 & -11.8 & -1.5 & -20.9 & -9.7 \\
\hline Health care & 9.7 & 10.3 & 4.6 & 4.3 & 0.8 & 0.1 & -0.1 & 1.1 \\
\hline Education & 6.8 & 6.7 & 2.1 & 1.3 & 1.6 & 1.0 & -0.3 & 1.1 \\
\hline Culture and science & 5.9 & 1.4 & 1.2 & -0.7 & -1.5 & -2.2 & 2.6 & 0.3 \\
\hline Banks and insurance & 7.1 & 5.1 & 2.1 & -1.1 & -9.0 & -2.1 & 0.2 & -3.0 \\
\hline Public administration and defense & 2.4 & 5.9 & 2.9 & 0.6 & -1.0 & -1.9 & -0.5 & 2.6 \\
\hline \multirow[t]{2}{*}{ FISIM $2 /$} & 18.7 & 5.0 & -14.4 & -2.6 & -2.0 & -1.2 & -0.6 & -1.6 \\
\hline & \multicolumn{8}{|c|}{ (Contribution to growth) } \\
\hline Industry & 2.8 & 2.7 & 2.2 & 1.8 & 3.0 & 2.5 & 2.5 & 2.9 \\
\hline Agriculture & -1.0 & 1.2 & 0.2 & 0.3 & 0.0 & -0.1 & 1.6 & 1.7 \\
\hline Construction & -0.2 & -0.2 & -0.3 & 0.7 & 0.2 & 0.7 & 1.4 & 0.8 \\
\hline Transport & 0.2 & 0.2 & 0.2 & 0.2 & 0.6 & 0.5 & 0.9 & 0.8 \\
\hline
\end{tabular}

Source: Ministry of Statistics and Analysis.

$1 /$ Current output is compared with previous output based on prices from the previous period.

2/ Undistributed imputed payments to financial intermediaries. 
Table 5. Belarus: Capital Investment by Sector, 1999-2003 1/ (In comparable prices)

\begin{tabular}{|c|c|c|c|c|c|}
\hline & 1999 & 2000 & 2001 & 2002 & 2003 \\
\hline & \multicolumn{5}{|c|}{ (Index, 1990=100) } \\
\hline Total & 51.2 & 52.2 & 50.4 & 53.7 & 61.9 \\
\hline Industry & 58.4 & 64.8 & 65.7 & 70.3 & 81.1 \\
\hline Agriculture & 11.3 & 12.2 & 8.5 & 9.9 & 12.4 \\
\hline Transport and communication & 107.8 & 95.1 & 117.7 & 135.0 & 166.0 \\
\hline Construction & 30.1 & 22.9 & 28.7 & 30.7 & 29.4 \\
\hline Residential construction & 66.9 & 69.4 & 60.4 & 54.1 & 57.7 \\
\hline \multirow[t]{2}{*}{ Other } & 70.0 & 69.5 & 63.3 & 73.0 & 84.6 \\
\hline & \multicolumn{5}{|c|}{ (Share of total) } \\
\hline Industry & 27.8 & 30.2 & 31.8 & 31.9 & 31.9 \\
\hline Agriculture & 6.4 & 6.8 & 4.9 & 5.3 & 5.8 \\
\hline Transport and communication & 15.5 & 13.4 & 17.2 & 18.5 & 19.8 \\
\hline Construction & 1.9 & 1.4 & 1.8 & 1.8 & 1.5 \\
\hline Residential construction & 25.7 & 26.1 & 23.5 & 19.8 & 18.3 \\
\hline Other & 22.7 & 22.1 & 20.8 & 22.7 & 22.7 \\
\hline \multicolumn{6}{|l|}{ Of which } \\
\hline Trade and catering & 3.0 & 2.6 & 3.0 & 3.7 & 3.2 \\
\hline Total & 100.0 & 100.0 & 100.0 & 100.0 & 100.0 \\
\hline \multicolumn{6}{|l|}{ Memorandum items: } \\
\hline Real gross investment (index, 1990=100) 2 & 50.9 & 58.4 & 59.5 & 61.0 & ... \\
\hline Gross investment (in percent of GDP) 2/ & 23.7 & 25.4 & 23.8 & 22.2 & $\ldots$ \\
\hline
\end{tabular}

Source: Ministry of Statistics and Analysis.

1/ Some figures may be inconsistent with the national accounts tables, as the data are based on surveys of industrial projects by branches of the economy.

2/ According to national accounts data. 
Table 6. Belarus: Industrial Production, 1999-2003

(Percentage change in comparable prices)

\begin{tabular}{lrrrrr}
\hline & 1999 & 2000 & 2001 & 2002 & 2003 \\
\hline Total & 10.3 & 7.8 & 5.9 & 4.5 & 6.8 \\
Of which: & & & & & \\
$\quad$ Power generation & 5.4 & -2.7 & 1.1 & 3.1 & 0.9 \\
$\quad$ Refineries & 1.9 & 15.9 & 4.9 & 12.1 & 7.2 \\
Chemicals and petrochemicals & 7.0 & 2.7 & 5.6 & 0.0 & 8.0 \\
Ferrous metallurgy & 0.0 & 10.0 & 7.4 & 6.2 & 6.2 \\
Machine building & 16.2 & 15.4 & 11.3 & 5.5 & 8.0 \\
Wood and paper & 16.0 & 6.1 & 7.4 & 6.5 & 7.3 \\
Construction materials & 1.5 & -2.0 & 4.6 & 7.2 & 11.7 \\
Light industry & 10.8 & 5.1 & 0.9 & -1.8 & 4.1 \\
Food-processing industries & 14.4 & 6.7 & 10.0 & 4.3 & 6.7 \\
\hline
\end{tabular}

Sources: Ministry of Statistics and Analysis; and IMF staff estimates. 


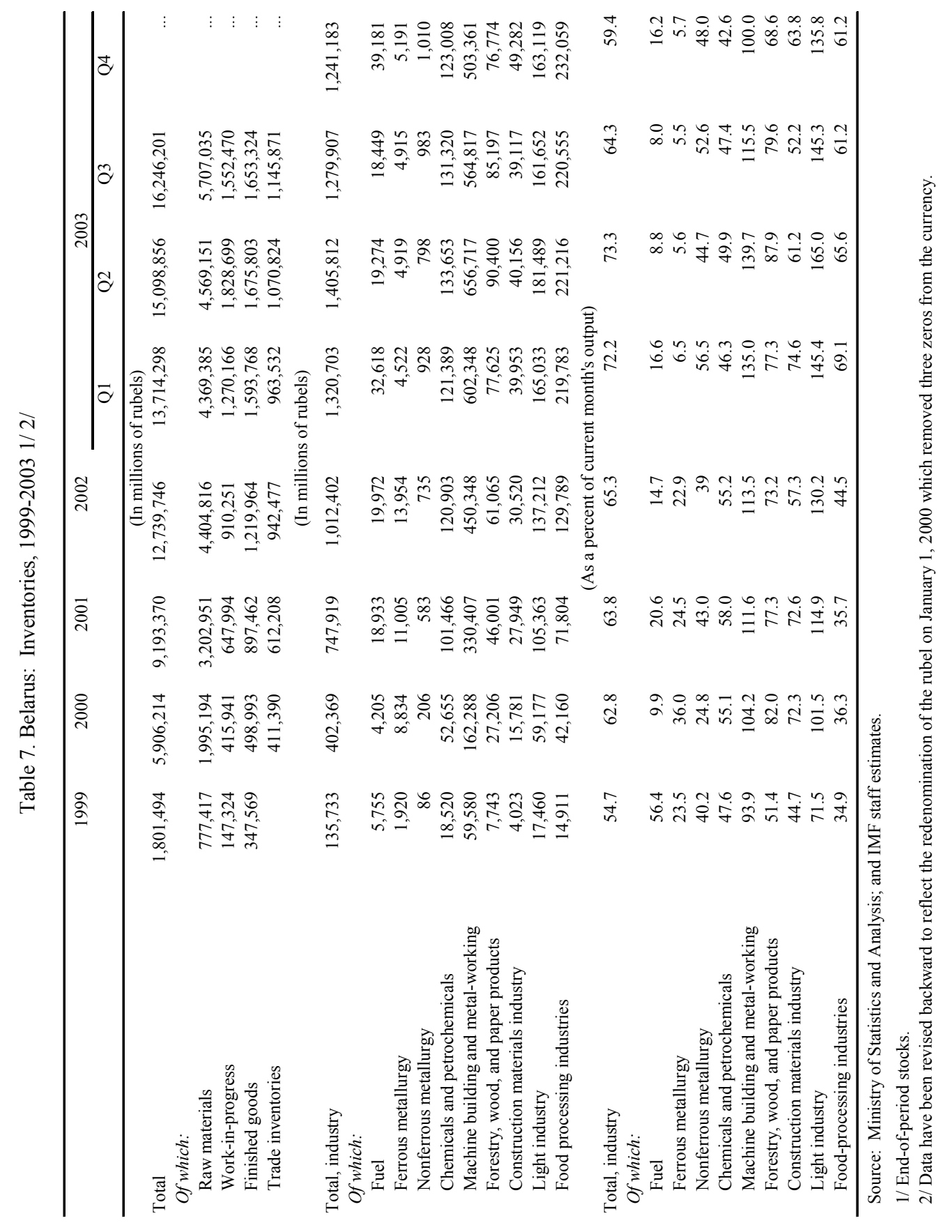


Table 8. Belarus: Agricultural Production, 1999-2003 1/

\begin{tabular}{|c|c|c|c|c|c|}
\hline & 1999 & 2000 & 2001 & 2002 & 2003 \\
\hline & \multicolumn{5}{|c|}{ (At current prices, in billions of rubels) } \\
\hline \multicolumn{6}{|l|}{$\begin{array}{l}\text { Agricultural output - total } \\
\text { Of which: }\end{array}$} \\
\hline Plant growing & 514 & 1,475 & 2,358 & 3,568 & 4,277 \\
\hline \multirow[t]{2}{*}{ Animal husbandry } & 422 & 1,259 & 2,181 & 2,953 & 3,159 \\
\hline & \multicolumn{5}{|c|}{ (At comparable prices, percentage change) } \\
\hline $\begin{array}{l}\text { Agricultural output - total } \\
\text { Of which: }\end{array}$ & -8.3 & $9,3^{2 /}$ & 1.8 & 0.7 & 6.8 \\
\hline Plant growing & -5.4 & 17.0 & 0.6 & 2.0 & 12.3 \\
\hline \multirow[t]{2}{*}{ Animal husbandry } & -10.4 & -2.3 & 3.7 & -1.0 & -0.8 \\
\hline & \multicolumn{5}{|c|}{ (In thousands of metric tons, unless otherwise indicated) } \\
\hline Grain & 3,645 & 4,856 & 5,153 & 5,990 & 5,449 \\
\hline Potatoes & 7,491 & 8,718 & 7,768 & 7,421 & 8,649 \\
\hline Flax & 21 & 37 & 32 & 26 & 41 \\
\hline Sugar beets & 1,187 & 1,474 & 1,682 & 1,146 & 1,920 \\
\hline Meat & 949 & 854 & 898 & 883 & 878 \\
\hline Milk & 4,741 & 4,490 & 4,834 & 4,773 & 4,702 \\
\hline Eggs & 3,395 & 3,288 & 3,144 & 2,923 & 2,810 \\
\hline \multicolumn{6}{|l|}{ Live animals (1,000 head) $3 /$} \\
\hline Cattle & 4,326 & 4,221 & 4,085 & 4,005 & 3,921 \\
\hline Pigs & 3,566 & 3,431 & 3,372 & 3,329 & 3,277 \\
\hline Sheep & 92 & 89 & 83 & 73 & 63 \\
\hline \multirow[t]{2}{*}{ Horses } & 221 & 217 & 209 & 202 & 192 \\
\hline & \multicolumn{5}{|c|}{ (Percentage change from previous year) } \\
\hline Grain & -24.5 & 33.2 & 6.1 & 16.2 & -9.0 \\
\hline Potatoes & -1.1 & 16.4 & -10.9 & -4.5 & 16.5 \\
\hline Flax & -41.7 & 76.2 & -13.5 & -18.7 & 57.7 \\
\hline Sugar beets & -16.9 & 24.2 & 14.1 & -31.9 & 67.5 \\
\hline Meat & -3.2 & -10.0 & 5.2 & -1.7 & -0.6 \\
\hline Milk & -9.4 & -5.3 & 7.7 & -1.3 & -1.5 \\
\hline Eggs & -2.5 & -3.2 & -4.4 & -7.0 & -4.0 \\
\hline \multicolumn{6}{|l|}{ Livestock 3/ } \\
\hline Cattle & -7.7 & -2.4 & -3.2 & -2.0 & -2.1 \\
\hline Pigs & -3.6 & -3.8 & -1.7 & -1.3 & -1.6 \\
\hline Sheep & -13.2 & -3.3 & -6.7 & -12.0 & -13.7 \\
\hline \multirow[t]{2}{*}{ Horses } & -3.5 & -1.8 & -3.7 & -3.3 & -5.0 \\
\hline & \multicolumn{5}{|c|}{ (Yield per hectare) } \\
\hline Grain & 15 & 19.4 & 19.9 & 24.7 & 24.2 \\
\hline Potatoes & 114 & 134 & 123 & 137 & 164 \\
\hline Flax & 3.1 & 4.8 & 4.2 & 4.4 & 6.1 \\
\hline Sugar beets & 227 & 292 & 313 & 228 & 275 \\
\hline
\end{tabular}

Sources: Ministry of Statistics and Analysis; and IMF staff estimates.

1/ Data have been revised backward to reflect the redenomination of the rubel on January 1, 2000 which removed three zeros from the currency.

$2 /$ Since 2000, in average prices of previous year.

3/ End-of-period stocks. 
Table 9. Belarus: Production and Consumption of Energy, 1999-2003

\begin{tabular}{|c|c|c|c|c|c|}
\hline & 1999 & 2000 & 2001 & 2002 & 2003 \\
\hline \multicolumn{6}{|l|}{ Production } \\
\hline Electricity (million kwh) & 26,516 & 26,101 & 25,063 & 26,455 & 26,615 \\
\hline Natural gas (million cubic meters) & 256 & 257 & 255 & 246 & 254 \\
\hline Crude oil (thousand tons) & 1,840 & 1,851 & 1,852 & 1,846 & 1,820 \\
\hline Gasoline (thousand tons) & 1,685 & 1,964 & 1,824 & 1,756 & 1,895 \\
\hline Diesel (thousand tons) & 3,398 & 3,847 & 3,880 & 4,606 & 4,912 \\
\hline Heavy fuel oil (thousand tons) & 4,305 & 4,627 & 4,408 & 5,099 & 4,790 \\
\hline \multicolumn{6}{|l|}{ Imports } \\
\hline Electricity (million kwh) 1/ & 10,192 & 9,975 & 10,989 & 10,068 & 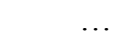 \\
\hline Natural gas (million cubic meters) & 16,565 & 17,115 & 17,266 & 17,578 & 18,113 \\
\hline Crude oil (thousand tons) & 9,900 & 12,010 & 11,913 & 14,024 & 14,885 \\
\hline Gasoline (thousand tons) & 55 & 38 & 9 & 12 & $652 /$ \\
\hline Diesel (thousand tons) & 90 & 236 & 63 & 75 & $2482 /$ \\
\hline Heavy fuel oil (thousand tons) & 354 & 440 & 108 & 232 & 717 \\
\hline \multicolumn{6}{|l|}{ Exports } \\
\hline Electricity (million kwh) 1/ & 3,029 & 2,764 & 2,718 & 3,513 & ... \\
\hline Crude oil (thousand tons) & 350 & 351 & 401 & 601 & 801 \\
\hline Gasoline (thousand tons) & 629 & 1,026 & 910 & 938 & 1,035 \\
\hline Diesel (thousand tons) & 1,761 & 2,339 & 2,298 & 3,270 & 3,493 \\
\hline Heavy fuel oil (thousand tons) & 2,006 & 3,076 & 2,621 & 3,694 & 4,063 \\
\hline \multicolumn{6}{|l|}{ Change in stocks } \\
\hline Natural gas (million cubic meters) & 6 & -197 & -112 & -100 & 81 \\
\hline Crude oil (thousand tons) & 48 & -53 & -49 & -32 & -134 \\
\hline Gasoline (thousand tons) & 16 & 11 & -39 & 73 & 27 \\
\hline Diesel (thousand tons) & 97 & 20 & 11 & -60 & 73 \\
\hline Heavy fuel oil (thousand tons) & 194 & 73 & 136 & 185 & 123 \\
\hline \multicolumn{6}{|l|}{ Consumption } \\
\hline Electricity (million kwh) & 33,679 & 33,312 & 33,334 & 33,010 & 33,228 \\
\hline \multicolumn{6}{|l|}{ Of which: } \\
\hline Industrial sector (million kwh) & 15,668 & 15,748 & 15,777 & 15,116 & $\ldots$ \\
\hline Natural gas (million cubic meters) & 16,827 & 17,175 & 17,409 & 17,724 & 18,448 \\
\hline \multicolumn{6}{|l|}{ Of which: } \\
\hline $\begin{array}{l}\text { For production of heating and } \\
\text { electricity (million meters) }\end{array}$ & 12,096 & 12,886 & 13,060 & 13,205 & 13,462 \\
\hline Crude oil (thousand tons) & 11,438 & 13,457 & 13,315 & 15,237 & 15,770 \\
\hline Gasoline (thousand tons) & 1,127 & 987 & 884 & 903 & 952 \\
\hline Diesel (thousand tons) & 1,824 & 1,764 & 1,655 & 1,671 & 1,740 \\
\hline Heavy fuel oil (thousand tons) & 2,847 & 2,064 & 2,031 & 1,821 & 1,567 \\
\hline \multicolumn{6}{|l|}{ Of which: } \\
\hline $\begin{array}{l}\text { For production of heating and } \\
\text { electricity (million meters) }\end{array}$ & 2,335 & 1,630 & 1,662 & 1,464 & 1,196 \\
\hline \multicolumn{6}{|l|}{ Losses in distribution } \\
\hline Electricity (million kwh) & 3,544 & 3,410 & 3,455 & 3,406 & $\cdots$ \\
\hline Natural gas (million cubic meters) & 103 & 229 & 202 & 197 & 208 \\
\hline
\end{tabular}

Source: Ministry of Statistics and Analysis.

1 / Includes transit. 
Table 10. Belarus: Change in Consumer and Producer Prices 2000-03

\begin{tabular}{|c|c|c|c|c|}
\hline & \multicolumn{2}{|c|}{ Consumer Prices: CPI 1/ } & \multicolumn{2}{|c|}{ Producer Prices: IPPI 2/ } \\
\hline & $\begin{array}{c}\text { Monthly percentage } \\
\text { change }\end{array}$ & $\begin{array}{c}\text { Year-on-year } \\
\text { percentage change }\end{array}$ & $\begin{array}{c}\text { Monthly percentage } \\
\text { change }\end{array}$ & $\begin{array}{c}\text { Year-on-year } \\
\text { percentage change }\end{array}$ \\
\hline \multicolumn{5}{|l|}{2000} \\
\hline January & 14.1 & 244 & 11.6 & 198 \\
\hline February & 9.3 & 230 & 10.3 & 178 \\
\hline March & 5.8 & 212 & 10.3 & 167 \\
\hline April & 5.1 & 205 & 6.4 & 167 \\
\hline May & 4.7 & 193 & 9.7 & 175 \\
\hline June & 6.1 & 190 & 10.1 & 188 \\
\hline July & 4.7 & 187 & 8.6 & 197 \\
\hline August & 3.6 & 178 & 9.3 & 201 \\
\hline September & 6.8 & 164 & 8.9 & 205 \\
\hline October & 5.2 & 143 & 6.6 & 197 \\
\hline November & 5.4 & 124 & 5.6 & 184 \\
\hline December & 5.1 & 108 & 5.6 & 168 \\
\hline \multicolumn{5}{|l|}{2001} \\
\hline January & 4.8 & 91 & 3.7 & 149 \\
\hline February & 3.9 & 81 & 3.4 & 134 \\
\hline March & 3.9 & 78 & 3.4 & 119 \\
\hline April & 3.3 & 75 & 3.1 & 112 \\
\hline May & 2.6 & 72 & 0.8 & 95 \\
\hline June & 2.1 & 65 & 1.3 & 80 \\
\hline July & 1.6 & 60 & 1.2 & 67 \\
\hline August & 0.8 & 56 & 1.9 & 56 \\
\hline September & 2.1 & 49 & 2.6 & 47 \\
\hline October & 3.6 & 47 & 3.6 & 43 \\
\hline November & 4.6 & 46 & 3.9 & 40 \\
\hline December & 5.5 & 46 & 4.6 & 39 \\
\hline \multicolumn{5}{|l|}{2002} \\
\hline January & 6.1 & 48 & 3.6 & 39 \\
\hline February & 3.6 & 48 & 3.3 & 39 \\
\hline March & 2.6 & 46 & 2.4 & 37 \\
\hline April & 2.9 & 45 & 2.4 & 36 \\
\hline May & 2.2 & 45 & 0.5 & 36 \\
\hline June & 1.4 & 44 & 1.9 & 37 \\
\hline July & 1.2 & 43 & 2.7 & 39 \\
\hline August & 1.1 & 43 & 6.9 & 46 \\
\hline September & 1.2 & 42 & 1.3 & 44 \\
\hline October & 1.7 & 40 & 2.2 & 42 \\
\hline November & 3.2 & 38 & 7.2 & 46 \\
\hline December & 3.2 & 35 & 2.0 & 43 \\
\hline \multicolumn{5}{|l|}{2003} \\
\hline January & 4.3 & 33 & 2.7 & 41 \\
\hline February & 1.9 & 30 & 5.3 & 44 \\
\hline March & 1.8 & 29 & 1.6 & 43 \\
\hline April & 2.0 & 28 & 1.0 & 41 \\
\hline May & 2.0 & 28 & 1.5 & 43 \\
\hline June & 1.8 & 28 & 0.8 & 41 \\
\hline July & 1.5 & 29 & 2.2 & 40 \\
\hline August & 0.2 & 28 & 2.0 & 34 \\
\hline September & 1.7 & 28 & 2.7 & 36 \\
\hline October & 1.9 & 29 & 2.0 & 36 \\
\hline November & 1.9 & 27 & 2.1 & 29 \\
\hline December & 2.0 & 25 & 1.6 & 29 \\
\hline \multicolumn{5}{|l|}{ Average } \\
\hline 2000 & 6.3 & 169 & 8.6 & 186 \\
\hline 2001 & 3.2 & 61 & 2.8 & 72 \\
\hline 2002 & 2.5 & 43 & 3.0 & 40 \\
\hline 2003 & 1.9 & 28 & 2.0 & 38 \\
\hline
\end{tabular}

Source: Ministry of Statistics and Analysis.

1/ The Consumer Price Index (CPI) uses weights from the previous year's Household Expenditure Survey.

2/ Industrial Production Price Index (IPPI) data are based on a corrected index formula (Laspeyres).

Since January 2001, the PPI has been compiled using the 1999 weights. 


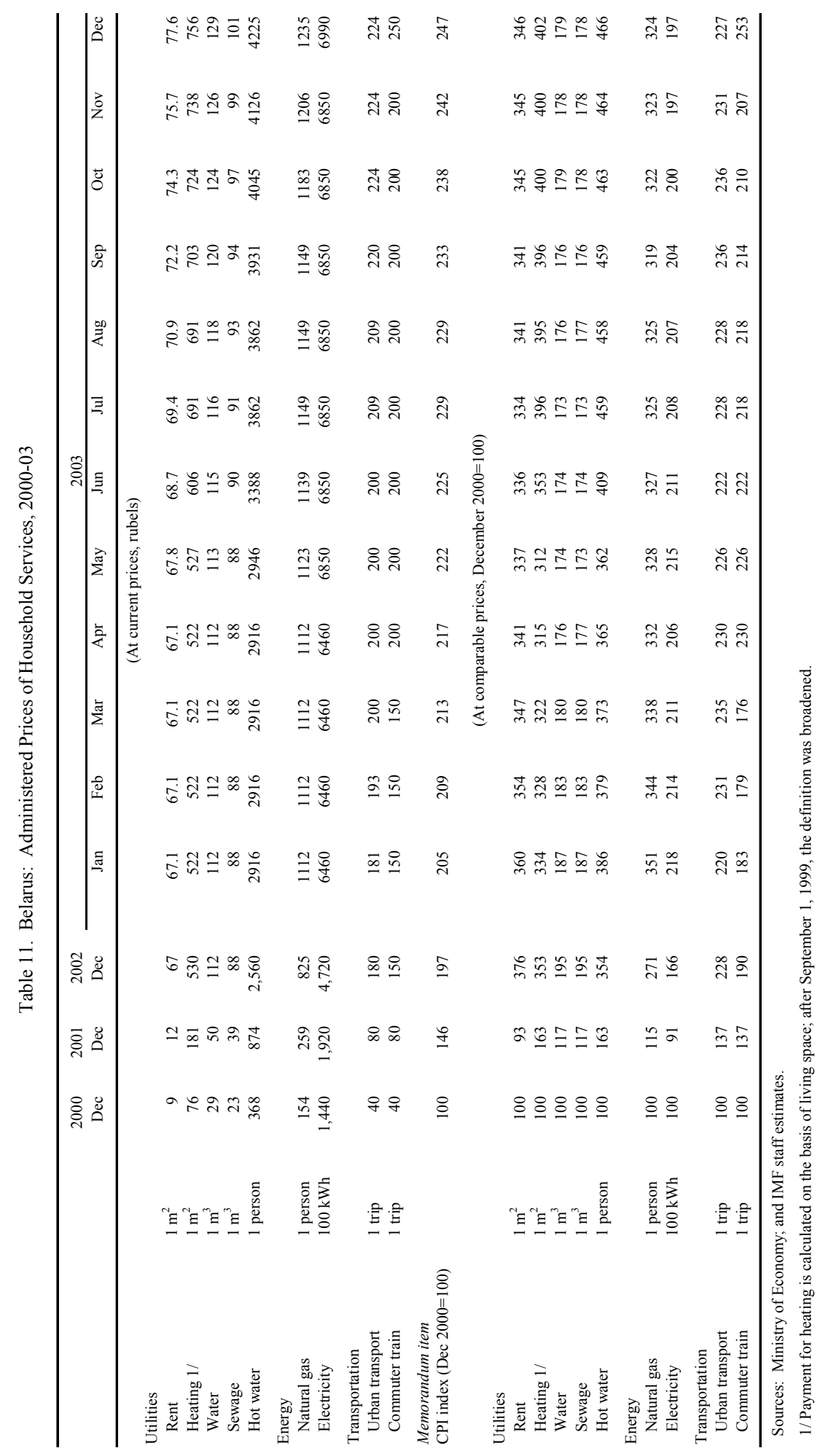




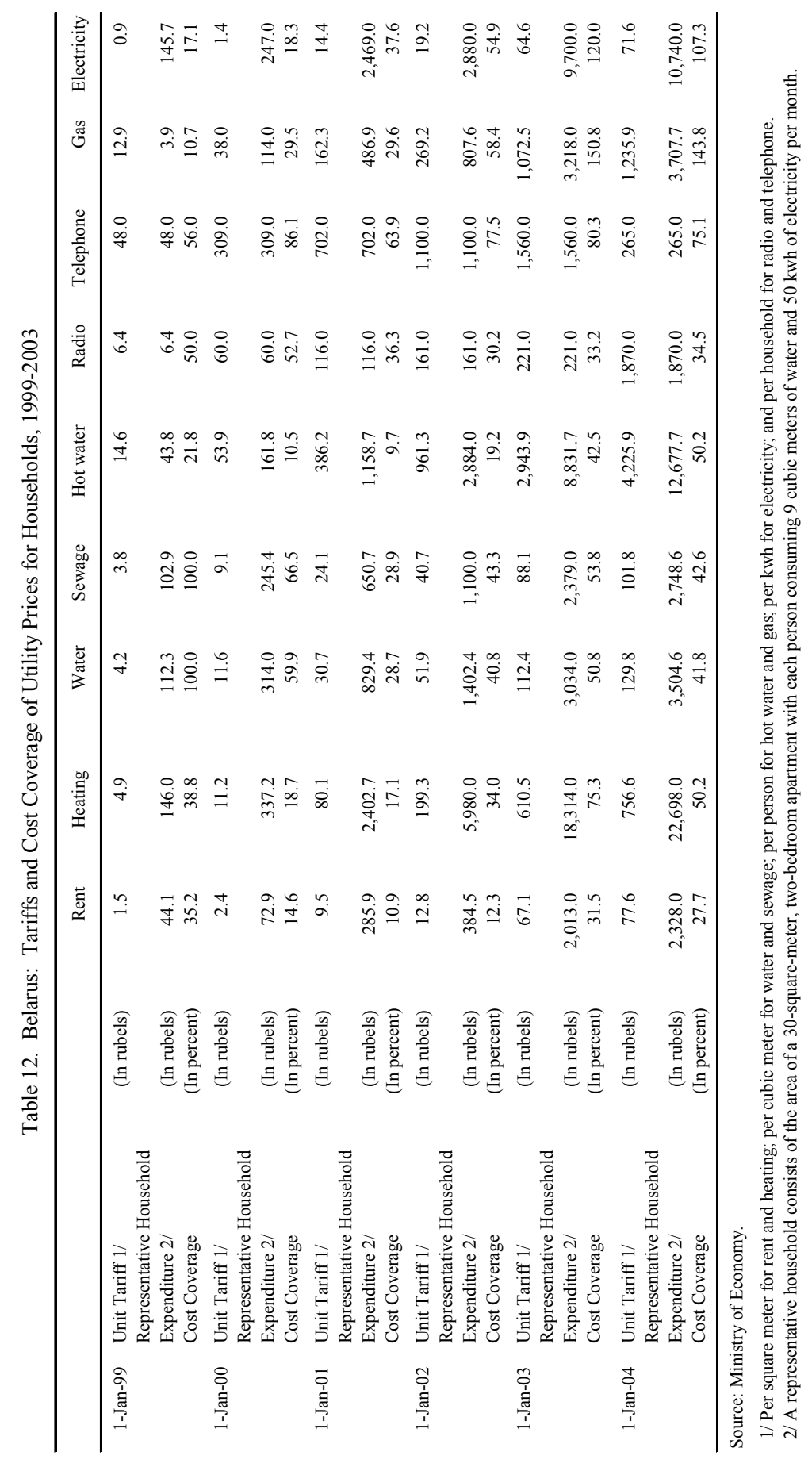


Table 13. Belarus: Average Monthly Wages, 1999-2003

(In thousands of rubels) 1/

\begin{tabular}{|c|c|c|c|c|c|}
\hline & 1999 & 2000 & 2001 & 2002 & 2003 \\
\hline & \multicolumn{5}{|c|}{ (Annual survey) $2 /$} \\
\hline Average & 19,581 & 58,916 & 123,021 & 189,232 & 253,527 \\
\hline \multicolumn{6}{|l|}{ Government } \\
\hline Construction & 25,238 & 74,660 & 151,122 & 228,579 & 327,289 \\
\hline Industry & 23,865 & 70,905 & 143,077 & 212,436 & 288,372 \\
\hline Forestry & 17,570 & 48,326 & 109,319 & 163,111 & 224,297 \\
\hline Agriculture & 11,282 & 36,774 & 78,218 & 113,090 & 137,660 \\
\hline \multicolumn{6}{|l|}{ Service-producing sectors } \\
\hline Banks and insurance & 39,928 & 111,650 & 225,171 & 351,081 & 449,457 \\
\hline Administration & 23,844 & 75,875 & 154,505 & 253,979 & 355,118 \\
\hline Information and computing services & 24,222 & 71,129 & 152,190 & 264,159 & 395,861 \\
\hline Transport & 21,240 & 63,858 & 132,795 & 213,403 & 302,679 \\
\hline Science & 23,753 & 73,975 & 157,461 & 239,246 & 328,874 \\
\hline Communication & 21,188 & 68,046 & 143,273 & 217,406 & 295,582 \\
\hline Housing and communal services & 18,180 & 56,179 & 122,048 & 192,695 & 255,125 \\
\hline Health & 15,290 & 45,898 & 106,101 & 170,239 & 226,073 \\
\hline Trade and catering & 16,397 & 47,332 & 95,522 & 146,115 & 193,195 \\
\hline Education & 14,577 & 44,869 & 101,497 & 163,724 & 214,119 \\
\hline Culture & 12,775 & 38,542 & 89,060 & 146,988 & 197,206 \\
\hline \multirow[t]{2}{*}{ Arts } & 12,634 & 40,542 & 95,481 & 152,165 & 208,292 \\
\hline & \multicolumn{5}{|c|}{ (Percentage change from the previous year) } \\
\hline Average & 322 & 201 & 109 & 54 & 32 \\
\hline \multicolumn{6}{|l|}{ Good-producing sectors } \\
\hline Construction & 335 & 196 & 102 & 51 & 37 \\
\hline Industry & 324 & 197 & 102 & 48 & 31 \\
\hline Forestry & 354 & 175 & 126 & 49 & 38 \\
\hline Agriculture & 306 & 226 & 113 & 45 & 24 \\
\hline \multicolumn{6}{|l|}{ Service-producing sectors } \\
\hline Banks and insurance & 341 & 180 & 102 & 56 & 33 \\
\hline Administration & 277 & 218 & 104 & 64 & 48 \\
\hline Information and computing services & 315 & 194 & 114 & 74 & 36 \\
\hline Transport & 333 & 201 & 108 & 61 & 34 \\
\hline Science & 356 & 211 & 113 & 52 & 39 \\
\hline Communication & 350 & 221 & 111 & 52 & 35 \\
\hline Housing and communal services & 335 & 209 & 117 & 58 & 26 \\
\hline Health & 319 & 200 & 131 & 60 & 33 \\
\hline Trade and catering & 303 & 189 & 102 & 53 & 26 \\
\hline Education & 300 & 208 & 126 & 61 & 31 \\
\hline Culture & 309 & 202 & 131 & 65 & 35 \\
\hline Arts & 313 & 221 & 136 & 59 & 35 \\
\hline \multicolumn{6}{|l|}{ Memorandum items: } \\
\hline Minimum wage (in thousands of rubels, end-of-period) & 1,450 & 3,600 & 10,000 & 20,330 & 47,910 \\
\hline Real average monthly wage index $(1991=100)$ & 82 & 92 & 119 & 128 & 134 \\
\hline Average monthly wage (U.S. dollars) & 77 & 89 & 90 & 107 & 123 \\
\hline
\end{tabular}

Source: Ministry of Statistics and Analysis; and IMF staff estimates.

1/ Data have been revised backward to reflect the redenomination of the rubel on January 1, 2000, which removed three zeros from the currency.

2/ Data for 2001 exclude non-public small enterprises. 


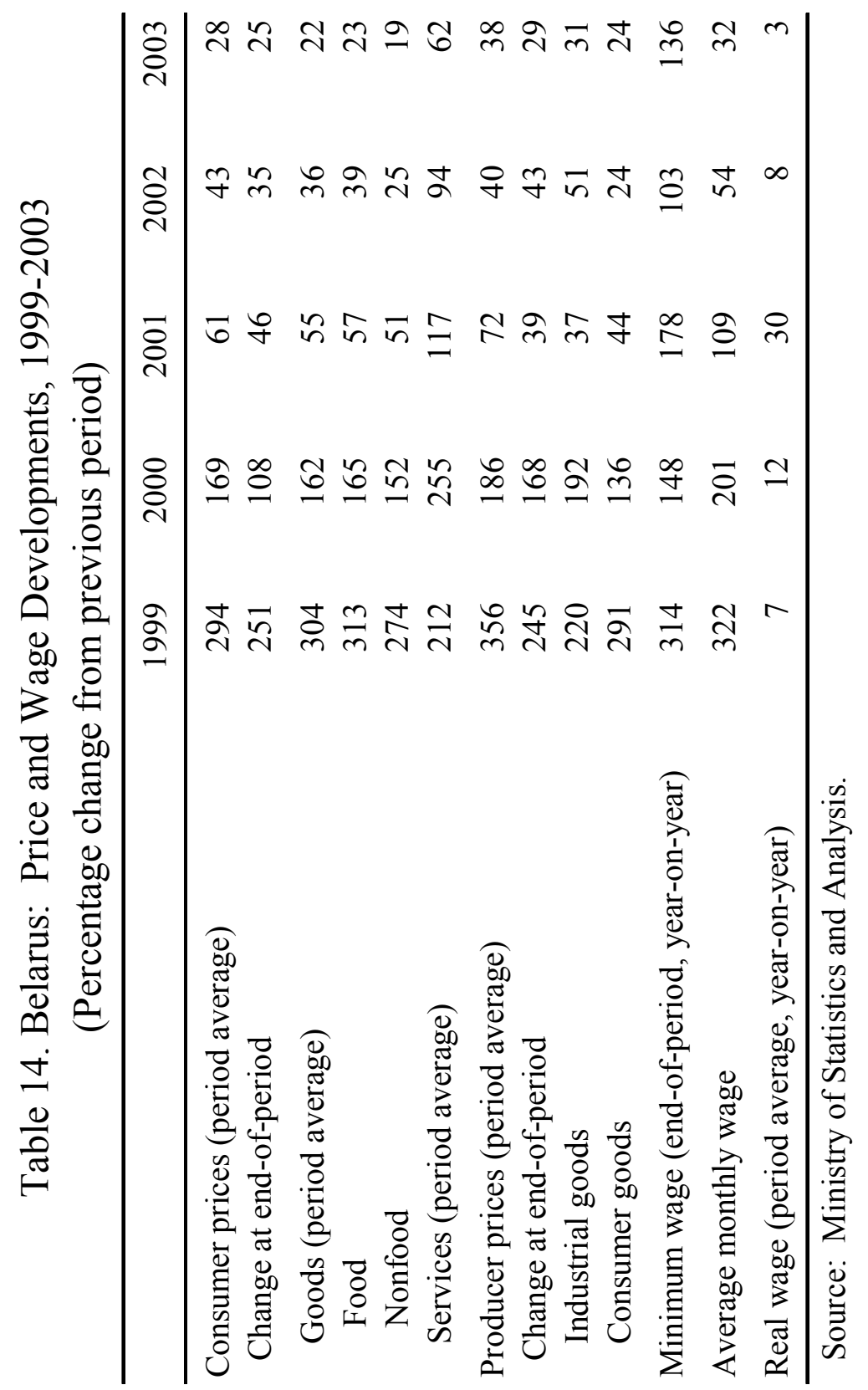


Table 15. Belarus: Money Income and Expenditures of the Population, 1999-2003

\begin{tabular}{|c|c|c|c|c|c|}
\hline & 1999 & 2000 & 2001 & 2002 & 2003 \\
\hline & \multicolumn{5}{|c|}{ (In millions of rubels) $1 /$} \\
\hline Money incomes & 1,872 & 6,011 & 12,478 & 19,154 & 25,421 \\
\hline $\begin{array}{l}\text { Salaries and wages of workers, and employees of } \\
\text { collective farms }\end{array}$ & 1,019 & 3,119 & 6,283 & 9,283 & 12,508 \\
\hline Revenues from sale of agricultural products & 39 & 95 & 162 & 199 & 265 \\
\hline Pensions, benefits, and scholarships & 335 & 1,079 & 2,360 & 3,622 & 4,604 \\
\hline Revenues from financial system $2 /$ & 53 & $\ldots$ & $\ldots$ & $\ldots$ & $\ldots$ \\
\hline $\begin{array}{l}\text { Revenues of population from officially } \\
\text { unaccounted business and other activities }\end{array}$ & 426 & 1,718 & 3,673 & 6,050 & 8,044 \\
\hline Expenditures & 1,842 & 5,935 & 12,341 & 19,085 & 25,282 \\
\hline Consumption of goods and payments for services & 1,597 & 4,566 & 9,082 & 14,142 & 18,226 \\
\hline $\begin{array}{l}\text { Taxes and other obligatory payments and } \\
\text { voluntary contributions }\end{array}$ & 165 & 522 & 1,074 & 1,611 & 2,442 \\
\hline $\begin{array}{l}\text { Accumulation of savings in deposit accounts, securities, } \\
\text { and foreign currency purchases }\end{array}$ & 80 & 847 & 2,185 & 3,332 & 4,614 \\
\hline Unallocated income & 30 & 76 & 137 & 69 & 139 \\
\hline & \multicolumn{5}{|c|}{ (As a percent of income) } \\
\hline $\begin{array}{l}\text { Salaries and wages of workers, and employees of } \\
\text { collective farms }\end{array}$ & 54.4 & 51.9 & 50.4 & 48.5 & 49.2 \\
\hline Revenues from sale of agricultural products & 2.1 & 1.6 & 1.3 & 1.1 & 1.1 \\
\hline Pensions, benefits, and scholarships & 17.9 & 17.9 & 18.9 & 18.9 & 18.1 \\
\hline Revenues from financial system $2 /$ & 2.9 & $\ldots$ & $\ldots$ & $\ldots$ & $\ldots$ \\
\hline $\begin{array}{l}\text { Revenues of population from officially } \\
\text { unaccounted business and other activities }\end{array}$ & 22.7 & 28.6 & 29.4 & 31.5 & 31.6 \\
\hline Saving rate $3 /$ & 5 & 15 & 19 & 19 & 20 \\
\hline
\end{tabular}

Source: Ministry of Statistics and Analysis; and IMF staff estimates.

1/ Data have been revised backward to reflect the redenomination of the rubel on January 1, 2000 removing three zeros from the currency.

2/ Beginning in January 2000, the item "Revenues from the financial system" is removed from the revenue side of the income balance. The reason for this is that credits received from the population are not current revenues, but equivalent to a change in financial assets. They are included in the item "Accumulation of savings in deposit accounts, securities, and foreign currency purchases" on the expenditure side.

3 / Including securities and foreign exchange deposits; expressed as a percentage of money income as defined for tax purposes. 
Table 16. Belarus: Labor Market Indicators, 1999-2003

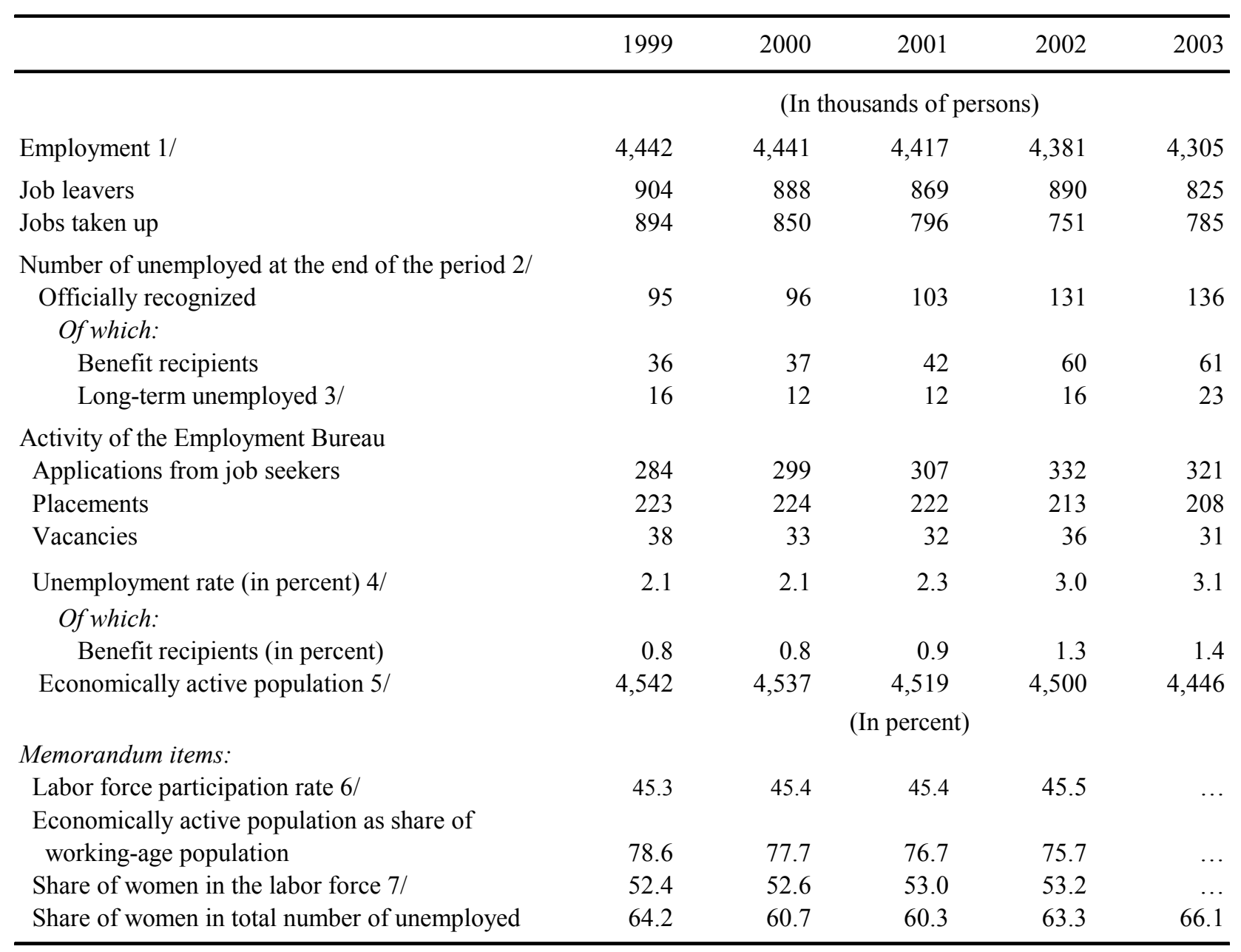

Source: Belarusian authorities; and IMF staff estimates.

1/ Average employment during the year for annual and quarterly data; end-of-period data for Q1 2000.

2/ Unemployed is any able-bodied person without a job other than: those engaged in business, those in education or training, or those serving in the armed forces. The definition was widened in 1993.

3/ Unemployed for more than one year.

4/ Number of registered unemployed expressed as a percentage of the economically active population, end of period.

5/ Working-age population comprises all people able to work older than 16 years and below the retirement age. The economically active population excludes, among others, students, housewives, and members of the armed forces, annual average

6/ Defined as the economically active population in percent of total population.

7/ Women on leave for maternity or caring for children under three years of age are exluded from the economically active population. 


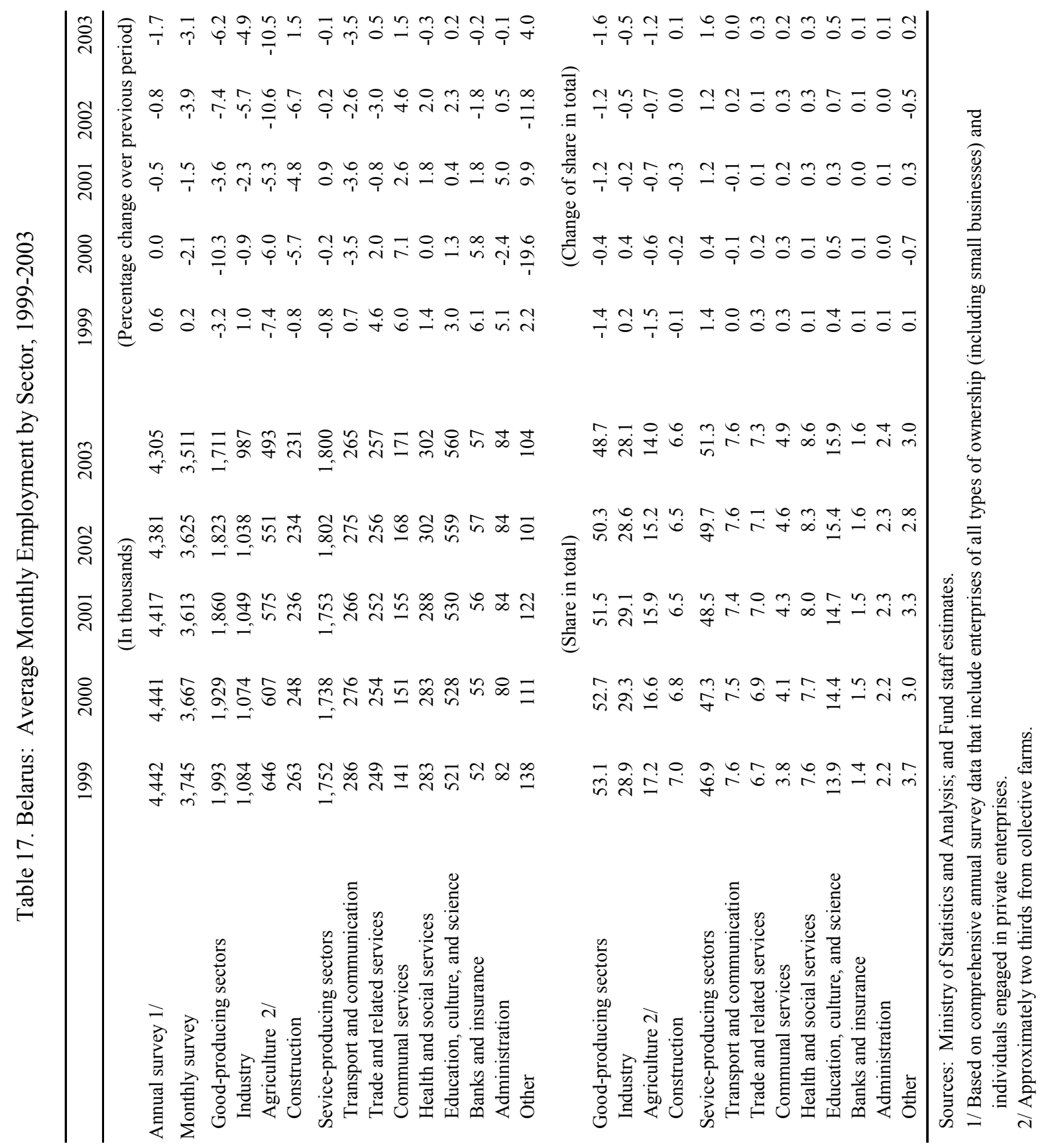


Table 18. Belarus: Enterprise Profits and Losses, 1999-2003

\begin{tabular}{|c|c|c|c|c|c|}
\hline & 1999 & 2000 & 2001 & 2002 & 2003 \\
\hline & \multicolumn{5}{|c|}{ (Profitability of enterprises) $1 /$} \\
\hline Total economy & 15.2 & 13.1 & 7.8 & 8.7 & 9.1 \\
\hline Industry & 17.1 & 15.8 & 10.9 & 10.5 & 12.0 \\
\hline \multicolumn{6}{|l|}{ Of which: } \\
\hline Power generation & 3.9 & 2.2 & 6.0 & 3.1 & 8.9 \\
\hline Refineries & 31.5 & 41.4 & 21.0 & 24.8 & 24.1 \\
\hline Ferrous metallurgy & 13.5 & 22.5 & 5.6 & 17.7 & 24.3 \\
\hline Chemicals & 27.1 & 25.8 & 12.8 & 12.8 & 16.1 \\
\hline Petrochemicals & 10.0 & 0.6 & 4.0 & -4.5 & 2.1 \\
\hline Machine building & 17.9 & 14.4 & 12.2 & 11.5 & 9.6 \\
\hline Wood and paper & 17.7 & 11.4 & 8.7 & 10.6 & 10.4 \\
\hline Construction materials & 8.1 & 5.2 & 4.6 & 7.1 & 9.5 \\
\hline Light industry & 22.6 & 14.3 & 6.1 & 5.0 & 4.3 \\
\hline Food industry & 13.4 & 9.2 & 8.1 & 5.4 & 6.0 \\
\hline \multirow[t]{2}{*}{ Agriculture 2/ } & 17.0 & 6.2 & -5.3 & -0.3 & -0.4 \\
\hline & \multicolumn{5}{|c|}{ (Number of loss-making enterprises) } \\
\hline Total & 1,935 & 2,613 & 3,909 & 4,082 & 3,078 \\
\hline \multicolumn{6}{|l|}{ Of which: } \\
\hline Industry & 209 & 456 & 696 & 798 & 550 \\
\hline \multirow[t]{2}{*}{ Agriculture 2/ } & 1,033 & 1,098 & 1,514 & 1,501 & 1,444 \\
\hline & \multicolumn{5}{|c|}{ (Share of loss-making enterprises per sector in percent) } \\
\hline Total economy & 16.9 & 22.3 & 33.4 & 34.9 & 27.2 \\
\hline Industry & 8.8 & 18.4 & 28.7 & 32.9 & 23.5 \\
\hline \multicolumn{6}{|l|}{ Of which: } \\
\hline Power generation & $\ldots$ & 25.0 & 12.5 & 12.5 & 12.5 \\
\hline Refineries & $\ldots$ & $\ldots$ & $\ldots$ & 33.3 & \\
\hline Ferrous metallurgy & 6.3 & $\ldots$ & 20.0 & 6.7 & 6.7 \\
\hline Chemicals & 7.7 & 11.3 & 27.1 & 32.2 & 24.1 \\
\hline Petrochemicals & 12.5 & 71.4 & 33.3 & 33.3 & 12.5 \\
\hline Machine building & 7.4 & 15.0 & 22.8 & 24.3 & 16.8 \\
\hline Wood and paper & 8.0 & 16.5 & 28.0 & 28.9 & 22.7 \\
\hline Construction materials & 19.9 & 31.7 & 49.2 & 45.5 & 18.1 \\
\hline Light industry & 13.1 & 30.7 & 46.4 & 47.9 & 34.9 \\
\hline Food industry & 2.9 & 12.1 & 20.7 & 38.2 & 30.0 \\
\hline
\end{tabular}

Source: Ministry of Statistics and Analysis.

1 / Profitability is defined as profits from sales as percentage of cost of sold products. With the exception of power generation, enterprises in this sample are those with an exclusively positive profitability.

2/ Includes state and collective farms. 
Table 19. Belarus: Interenterprise Arrears and the Barter Economy, 1999-2003

\begin{tabular}{|c|c|c|c|c|c|c|c|c|c|c|}
\hline & 1999 & 2000 & 2001 & 2002 & 2003 & 1999 & 2000 & 2001 & 2002 & 2003 \\
\hline & \multicolumn{5}{|c|}{ (In billions of rubels) $1 /$} & \multicolumn{5}{|c|}{ (In percent of GDP) } \\
\hline \multicolumn{11}{|l|}{ Overdue accounts receivable } \\
\hline Total & 419 & 1,658 & 3,067 & 3,943 & 4,185 & 13.9 & 18.1 & 17.9 & 15.1 & 11.6 \\
\hline Industry & 199 & 843 & 1,554 & 1,860 & 2,132 & 6.6 & 9.2 & 9.0 & 7.1 & 5.9 \\
\hline Agriculture & 6 & 22 & 38 & 68 & 105 & 0.2 & 0.2 & 0.2 & 0.3 & 0.3 \\
\hline Construction & 9 & 39 & 80 & 110 & 148 & 0.3 & 0.4 & 0.5 & 0.4 & 0.4 \\
\hline Transport & 95 & 331 & 521 & 698 & 595 & 3.1 & 3.6 & 3.0 & 2.7 & 1.7 \\
\hline Communications & 1 & 3 & 8 & 15 & 17 & 0.0 & 0.0 & 0.0 & 0.1 & 0.0 \\
\hline Trade and public catering & 3 & 14 & 43 & 89 & 186 & 0.1 & 0.2 & 0.3 & 0.3 & 0.5 \\
\hline Supply and sales & 21 & 83 & 126 & 240 & 316 & 0.7 & 0.9 & 0.7 & 0.9 & 0.9 \\
\hline Housing and communal services & 85 & 319 & 625 & 768 & 597 & 2.8 & 3.5 & 3.6 & 2.9 & 1.7 \\
\hline \multicolumn{11}{|l|}{ Overdue accounts payable 2/ } \\
\hline Total & 555 & 2,043 & 3,636 & 4,622 & 4,922 & 18.3 & 22.4 & 21.2 & 17.7 & 13.7 \\
\hline Industry & 311 & 1,198 & 1,872 & 2,550 & 2,495 & 10.3 & 13.1 & 10.9 & 9.8 & 6.9 \\
\hline Agriculture & 63 & 235 & 593 & 660 & 1,018 & 2.1 & 2.6 & 3.5 & 2.5 & 2.8 \\
\hline Construction & 8 & 32 & 63 & 109 & 129 & 0.2 & 0.3 & 0.4 & 0.4 & 0.4 \\
\hline Transport & 66 & 176 & 344 & 275 & 299 & 2.2 & 1.9 & 2.0 & 1.1 & 0.8 \\
\hline Communications & 3 & 12 & 13 & 3 & 4 & 0.1 & 0.1 & 0.1 & 0.0 & 0.0 \\
\hline Trade and public catering & 14 & 70 & 125 & 201 & 281 & 0.4 & 0.8 & 0.7 & 0.8 & 0.8 \\
\hline Supply and sales & 16 & 42 & 66 & 125 & 143 & 0.5 & 0.5 & 0.4 & 0.5 & 0.4 \\
\hline Housing and communal services & 74 & 274 & 521 & 656 & 553 & 2.5 & 3.0 & 3.0 & 2.5 & 1.5 \\
\hline \multicolumn{11}{|l|}{ Barter transactions } \\
\hline Total & 1,133 & 3,836 & 7,526 & 9,230 & 9,664 & 37.4 & 42.0 & 43.8 & 35.3 & 26.9 \\
\hline Industry & 911 & 2,936 & 5,552 & 6,604 & 6,528 & 30.1 & 32.1 & 32.3 & 25.3 & 18.2 \\
\hline Agriculture & 54 & 182 & 370 & 567 & 921 & 1.8 & 2.0 & 2.2 & 2.2 & 2.6 \\
\hline Construction & 34 & 99 & 264 & 322 & 394 & 1.1 & 1.1 & 1.5 & 1.2 & 1.1 \\
\hline Transport & 11 & 24 & 49 & 71 & 120 & 0.4 & 0.3 & 0.3 & 0.3 & 0.3 \\
\hline Communications & 2 & 8 & 17 & 53 & 76 & 0.1 & 0.1 & 0.1 & 0.2 & 0.2 \\
\hline Trade and public catering & 46 & 102 & 211 & 367 & 389 & 1.5 & 1.1 & 1.2 & 1.4 & 1.1 \\
\hline Supply and sales & 40 & 57 & 120 & 192 & 207 & 1.3 & 0.6 & 0.7 & 0.7 & 0.6 \\
\hline Housing and communal services & 29 & 368 & 814 & 944 & 881 & 1.0 & 4.0 & 4.7 & 3.6 & 2.5 \\
\hline \multicolumn{11}{|l|}{ Memorandum item: } \\
\hline Gross domestic product & 3,026 & 9,134 & 17,173 & 26,138 & 35,930 & $\cdots$ & $\ldots$ & $\ldots$ & $\ldots$ & $\cdots$ \\
\hline
\end{tabular}

Sources: Ministry of Statistics and Analysis.

1/ Data have been revised backward to reflect the redenomination of the rubel on January 1, 2000 which removed three zeros from the currency.

2/ Overdue accounts payable only include overdue payments to the republican and local governments, workers, and to other entities for goods and services. 


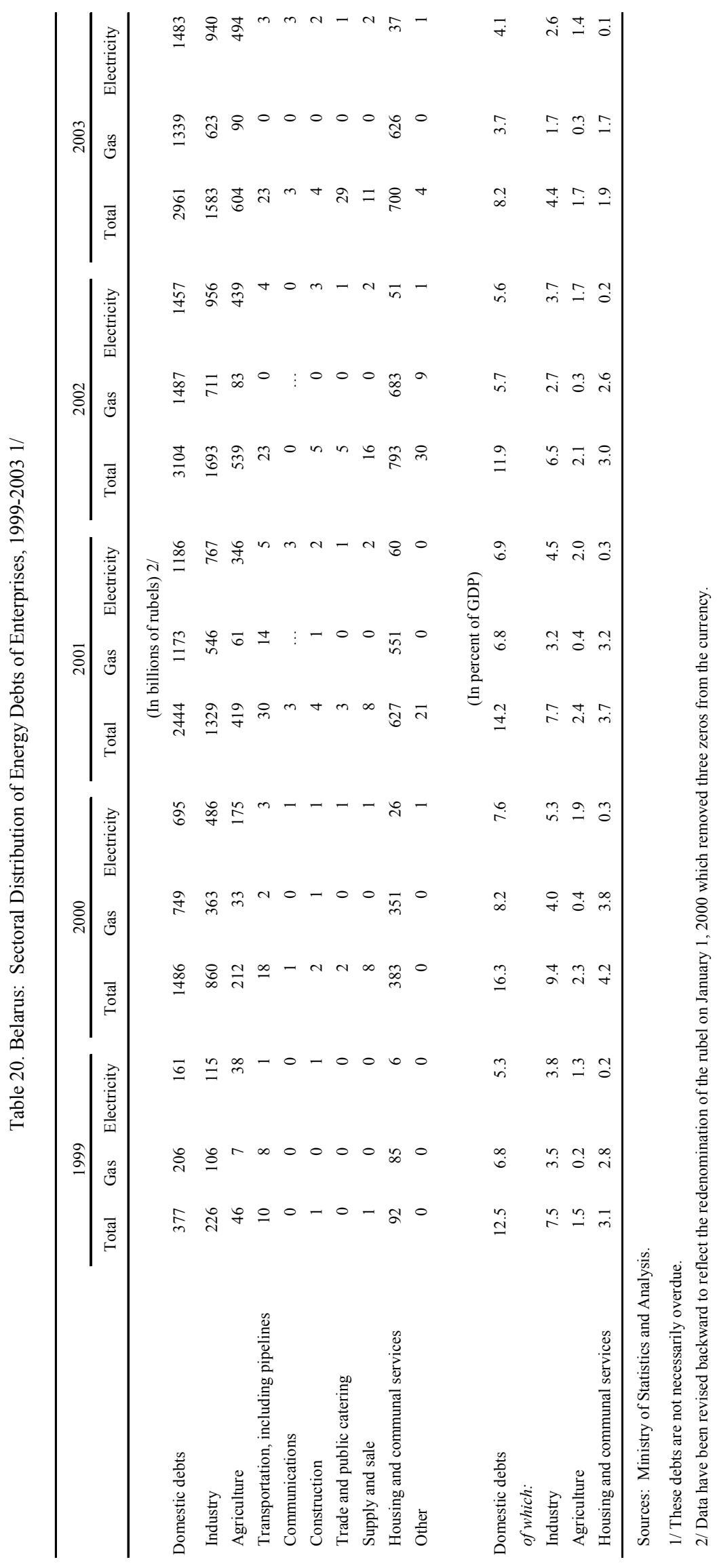


Table 21. Belarus: General Government Operations 1999-2003 (In millions of rubels) 1/

\begin{tabular}{|c|c|c|c|c|c|}
\hline & 1999 & 2000 & 2001 & 2002 & $\begin{array}{r}2003 \\
\text { Prelim } \\
\end{array}$ \\
\hline \multicolumn{6}{|l|}{ 1. Republican and local budgets } \\
\hline Revenue, excl.privatization & $1,052,385$ & $3,173,237$ & $5,734,597$ & $8,208,756$ & $12,154,223$ \\
\hline Current tax revenue & 805,456 & $2,433,564$ & $4,389,113$ & $6,263,381$ & $9,333,644$ \\
\hline Direct taxes on income and profits, $\mathrm{o} / \mathrm{w}$ : & 235,749 & 716,609 & $1,315,857$ & $1,684,505$ & $2,368,974$ \\
\hline Personal income tax & 89,288 & 277,110 & 534,233 & 773,057 & $1,024,400$ \\
\hline Profit tax & 141,694 & 395,083 & 636,850 & 643,339 & 934,427 \\
\hline Enterprise income tax & 4,205 & 44,416 & 94,200 & 136,839 & 173,255 \\
\hline Taxes on wage fund & 47,232 & 73,092 & 154,542 & 220,359 & 273,195 \\
\hline Taxes on goods and services, $\mathrm{o} / \mathrm{w}$ : & 424,418 & $1,336,006$ & $2,283,822$ & $3,311,709$ & $4,837,192$ \\
\hline VAT & 261,992 & 817,837 & $1,446,701$ & $2,165,127$ & $2,894,757$ \\
\hline Excises & 99,687 & 253,873 & 442,005 & 592,029 & 837,782 \\
\hline Property tax & 18,051 & 93,200 & 237,377 & 390,477 & 728,784 \\
\hline Current non-tax revenues & 59,934 & 111,834 & 224,510 & 382,952 & 596,276 \\
\hline Capital revenue & 9,766 & 33,461 & 58,962 & 449,198 & 51,478 \\
\hline Revenue from sales of assets & 9,529 & 32,713 & 58,826 & 448,830 & 49,706 \\
\hline Revenue of republican budgetary funds, o/w: & 89,754 & 589,878 & 908,330 & $1,275,492$ & $1,886,175$ \\
\hline Agriculture support fund & 48,202 & 300,942 & 467,067 & 601,236 & 899,977 \\
\hline Road fund & 32,429 & 177,812 & 374,019 & 517,752 & 725,026 \\
\hline Revenue of local budgetary funds & 86,596 & 76,460 & 124,721 & 194,664 & 287,437 \\
\hline Expenditure & $1,142,843$ & $3,236,393$ & $6,023,461$ & $8,681,144$ & $12,646,135$ \\
\hline Republican and local administrations & 29,823 & 87,661 & 168,081 & 251,565 & 381,539 \\
\hline Defense & 29,587 & 89,444 & 183,766 & 259,859 & 376,898 \\
\hline Law, order and security & 54,006 & 165,279 & 333,251 & 461,023 & 654,083 \\
\hline Agriculture & 37,254 & 87,349 & 146,435 & 178,782 & 520,308 \\
\hline Housing and communal services & 81,388 & 248,631 & 450,085 & 612,445 & 940,867 \\
\hline Emergency funds, Chernobyl & 48,611 & 110,917 & 228,702 & 307,028 & 376,378 \\
\hline Education & 185,708 & 562,129 & $1,109,509$ & $1,738,099$ & $2,343,119$ \\
\hline Health, sports and physical education & 150,841 & 455,031 & 872,523 & $1,270,067$ & $1,809,793$ \\
\hline Servicing of state debt & 22,976 & 82,467 & 124,861 & 154,203 & 175,696 \\
\hline Other & 273,508 & 619,856 & $1,157,884$ & $1,773,224$ & $2,601,306$ \\
\hline Capital investment & 67,407 & 169,926 & 252,342 & 323,604 & 533,431 \\
\hline Expenditure of budgetary funds, o/w: & 161,736 & 557,706 & 996,022 & $1,351,245$ & $1,932,718$ \\
\hline Agriculture support fund & 43,390 & 292,128 & 477,619 & 562,074 & 877,384 \\
\hline Road fund & 69,953 & 170,527 & 343,967 & 495,703 & 639,477 \\
\hline Balance & $-90,458$ & $-63,156$ & $-288,864$ & $-472,388$ & $-491,912$ \\
\hline \multicolumn{6}{|l|}{ 2. Social Protection Fund } \\
\hline Revenue (incl. from State Budget) & 325,767 & $1,017,960$ & $1,983,946$ & $3,055,027$ & $3,977,828$ \\
\hline Own revenue & 317,534 & $1,009,948$ & $1,978,904$ & $2,985,207$ & $3,921,154$ \\
\hline $\mathrm{o} / \mathrm{w}$ transfers from the budget & 8,233 & 8,012 & 5,043 & 69,820 & 56,674 \\
\hline Expenditure & 297,011 & 961,662 & $2,020,590$ & $3,061,403$ & $3,987,823$ \\
\hline Balance & 28,757 & 56,298 & $-36,644$ & $-6,376$ & $-9,995$ \\
\hline \multicolumn{6}{|l|}{ 3. Consolidated budget } \\
\hline Revenue & $1,369,919$ & $4,183,185$ & $7,713,501$ & $11,193,963$ & $16,075,327$ \\
\hline Tax revenue & $1,122,990$ & $3,443,512$ & $6,368,017$ & $9,248,588$ & $13,254,798$ \\
\hline Expenditure & $1,431,621$ & $4,190,043$ & $8,039,008$ & $11,672,727$ & $16,577,284$ \\
\hline Balance & $-61,702$ & $-6,858$ & $-325,507$ & $-478,764$ & $-501,907$ \\
\hline Statistical discrepancy & 3,292 & 1,945 & $-29,533$ & 114,284 & 63,828 \\
\hline 4. Financing (cash) & 64,993 & 8,803 & 295,974 & 593,048 & 565,735 \\
\hline Privatization & 2,518 & 7,794 & 12,492 & 427,353 & 35,819 \\
\hline Foreign financing, net (excl. IMF) & $-8,617$ & $-14,032$ & 19,701 & 29,164 & $-49,948$ \\
\hline Domestic financing & 71,092 & 15,041 & 263,782 & 136,532 & 579,865 \\
\hline
\end{tabular}

Sources: Belarusian Ministry of Finance, and Fund staff estimates.

1/ Data have been revised backward to reflect the redenomination of the rubel on January 1, 2000. 
Table 22. Belarus: General Government Operations 1999-2003 (In percent of GDP) 1/

\begin{tabular}{|c|c|c|c|c|c|}
\hline & 1999 & 2000 & 2001 & 2002 & $\begin{array}{r}2003 \\
\text { Prelim. } \\
\end{array}$ \\
\hline \multicolumn{6}{|l|}{ 1. Republican and local budgets } \\
\hline Revenue, excl.privatization & 34.8 & 34.7 & 33.4 & 31.4 & 33.8 \\
\hline Current tax revenue & 26.6 & 26.6 & 25.6 & 24.0 & 26.0 \\
\hline Direct taxes on income and profits, $\mathrm{o} / \mathrm{w}$ : & 7.8 & 7.8 & 7.7 & 6.4 & 6.6 \\
\hline Personal income tax & 3.0 & 3.0 & 3.1 & 3.0 & 2.9 \\
\hline Profit tax & 4.7 & 4.3 & 3.7 & 2.5 & 2.6 \\
\hline Enterprise income tax & 0.1 & 0.5 & 0.5 & 0.5 & 0.5 \\
\hline Taxes on wage fund & 1.6 & 0.8 & 0.9 & 0.8 & 0.8 \\
\hline Taxes on goods and services, o/w: & 14.0 & 14.6 & 13.3 & 12.7 & 13.5 \\
\hline VAT & 8.7 & 9.0 & 8.4 & 8.3 & 8.1 \\
\hline Excises & 3.3 & 2.8 & 2.6 & 2.3 & 2.3 \\
\hline Property tax & 0.6 & 1.0 & 1.4 & 1.5 & 2.0 \\
\hline Current non-tax revenues & 2.0 & 1.2 & 1.3 & 1.5 & 1.7 \\
\hline Capital revenue & 0.3 & 0.4 & 0.3 & 1.7 & 0.1 \\
\hline Revenue from sales of assets & 0.3 & 0.4 & 0.3 & 1.7 & 0.1 \\
\hline Revenue of republican budgetary funds, o/w: & 3.0 & 6.5 & 5.3 & 4.9 & 5.2 \\
\hline Agriculture support fund & 1.6 & 3.3 & 2.7 & 2.3 & 2.5 \\
\hline Road fund & 1.1 & 1.9 & 2.2 & 2.0 & 2.0 \\
\hline Revenue of local budgetary funds & 2.9 & 0.8 & 0.7 & 0.7 & 0.8 \\
\hline Expenditure & 37.8 & 35.4 & 35.1 & 33.2 & 35.2 \\
\hline Republican and local administrations & 1.0 & 1.0 & 1.0 & 1.0 & 1.1 \\
\hline Defense & 1.0 & 1.0 & 1.1 & 1.0 & 1.0 \\
\hline Law, order and security & 1.8 & 1.8 & 1.9 & 1.8 & 1.8 \\
\hline Agriculture & 1.2 & 1.0 & 0.9 & 0.7 & 1.4 \\
\hline Housing and communal services & 2.7 & 2.7 & 2.6 & 2.3 & 2.6 \\
\hline Emergency funds, Chernobyl & 1.6 & 1.2 & 1.3 & 1.2 & 1.0 \\
\hline Education & 6.1 & 6.2 & 6.5 & 6.6 & 6.5 \\
\hline Health, sports and physical education & 5.0 & 5.0 & 5.1 & 4.9 & 5.0 \\
\hline Servicing of state debt & 0.8 & 0.9 & 0.7 & 0.6 & 0.5 \\
\hline Other & 9.0 & 6.8 & 6.7 & 6.8 & 7.2 \\
\hline Capital investment & 2.2 & 1.9 & 1.5 & 1.2 & 1.5 \\
\hline Expenditure of budgetary funds, o/w: & 5.3 & 6.1 & 5.8 & 5.2 & 5.4 \\
\hline Agriculture support fund & 1.4 & 3.2 & 2.8 & 2.2 & 2.4 \\
\hline Road fund & 2.3 & 1.9 & 2.0 & 1.9 & 1.8 \\
\hline Balance & -3.0 & -0.7 & -1.7 & -1.8 & -1.4 \\
\hline \multicolumn{6}{|l|}{ 2. Social Protection Fund } \\
\hline Revenue (incl. from State Budget) & 10.8 & 11.1 & 11.6 & 11.7 & 11.1 \\
\hline Own revenue & 10.5 & 11.1 & 11.5 & 11.4 & 10.9 \\
\hline $\mathrm{o} / \mathrm{w}$ transfers from the budget & 0.3 & 0.1 & 0.0 & 0.3 & 0.2 \\
\hline Expenditure & 9.8 & 10.5 & 11.8 & 11.7 & 11.1 \\
\hline Balance & 1.0 & 0.6 & -0.2 & 0.0 & 0.0 \\
\hline \multicolumn{6}{|l|}{ 3. Consolidated budget } \\
\hline Revenue & 45.3 & 45.8 & 44.9 & 42.8 & 44.7 \\
\hline Tax revenue & 37.1 & 37.7 & 37.1 & 35.4 & 36.9 \\
\hline Expenditure & 47.3 & 45.9 & 46.8 & 44.7 & 46.1 \\
\hline Balance & -2.0 & -0.1 & -1.9 & -1.8 & -1.4 \\
\hline Statistical discrepancy & 0.1 & 0.0 & -0.2 & 0.4 & 0.2 \\
\hline 4. Financing (cash) & 2.1 & 0.1 & 1.7 & 2.3 & 1.6 \\
\hline Privatization & 0.1 & 0.1 & 0.1 & 1.6 & 0.1 \\
\hline Foreign financing, net (excl. IMF) & -0.3 & -0.2 & 0.1 & 0.1 & -0.1 \\
\hline Domestic financing & 2.3 & 0.2 & 1.5 & 0.5 & 1.6 \\
\hline
\end{tabular}

Sources: Belarusian Ministry of Finance, and Fund staff estimates.

1/ Data have been revised backward to reflect the redenomination of the rubel on January 1, 2000. 


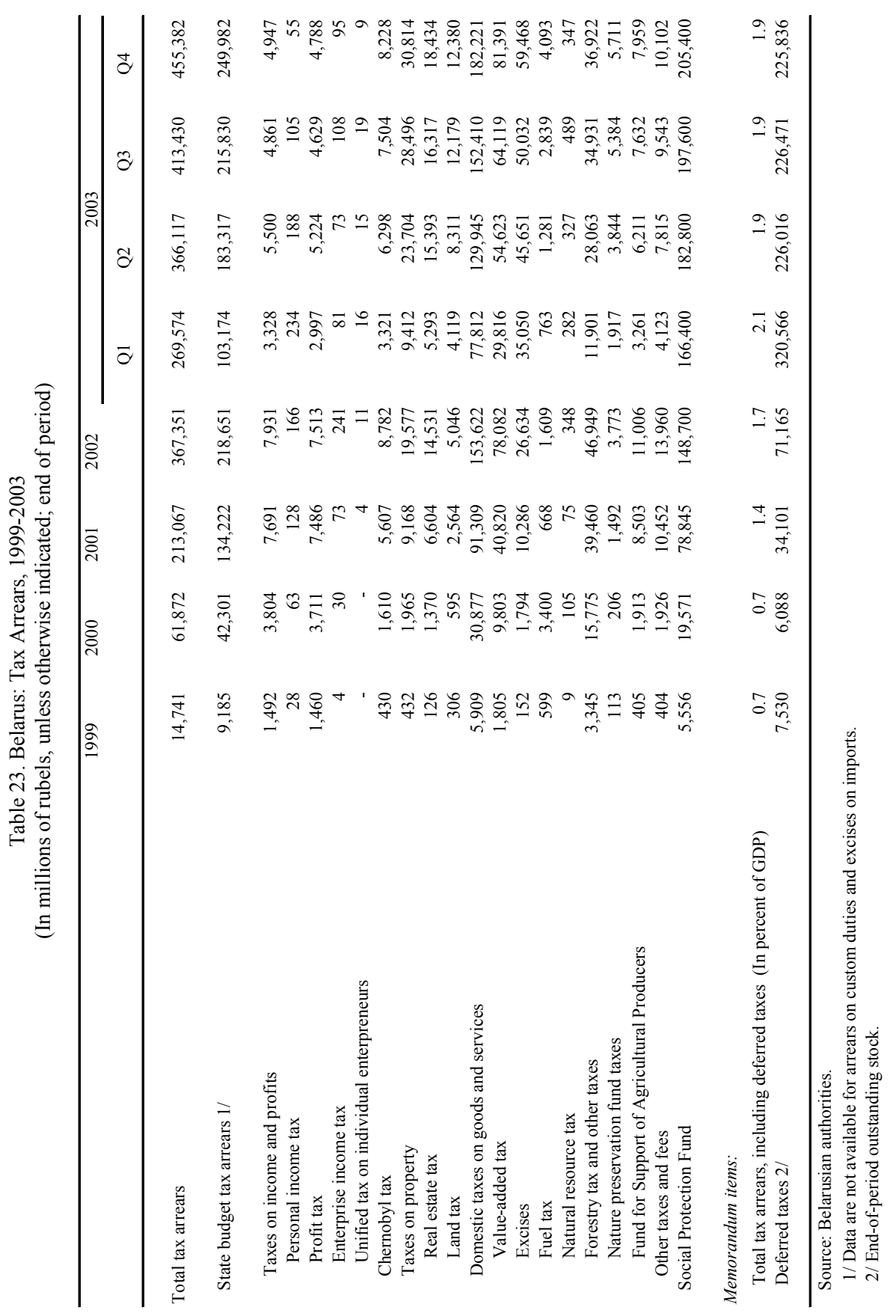


Table 24. Belarus: General Government Debt, 1999-2003 (In billions of rubels, unless otherwise indicated; end-of-period)

\begin{tabular}{lrrrrr}
\hline & 1999 & 2000 & 2001 & 2002 & 2003 \\
\hline Total debt, net & 445 & 1,167 & 1,557 & 1,847 & 2,589 \\
$\quad$ Domestic debt, net 1/ & 162 & 209 & 351 & 286 & 1,001 \\
of which: & & & & & \\
$\quad$ Republican government (net) & 148 & 222 & 329 & 370 & 1,051 \\
$\quad$ Local government (net) & 0.4 & -38 & -54 & -107 & -74 \\
$\quad$ T-bills held by nonbanks & 14 & 26 & 76 & 23 & 24 \\
Foreign debt 2/ & 283 & 958 & 1,206 & 1,561 & 1,588 \\
Memorandum items: & & & & & \\
$\quad$ Domestic debt (in percent of GDP) 3/ & 5.4 & 2.3 & 2.0 & 1.1 & 2.8 \\
Foreign debt (in millions of U.S. dollars) 2/ & 886 & 812 & 763 & 813 & 736 \\
Exchange rate (in rubels per U.S. dollar) & 319 & 1,180 & 1,580 & 1,920 & 2,156 \\
Nominal GDP & 3,026 & 9,134 & 17,173 & 26,138 & 35,930 \\
\hline
\end{tabular}

Sources: Belarusian authorities; and Fund staff estimates.

1/ The data are derived from NBB balance sheets.

$2 /$ As recorded in the balance of payments under public and publicly guaranteed debt, including IMF.

3 / In percent of GDP during the previous 12 months. 


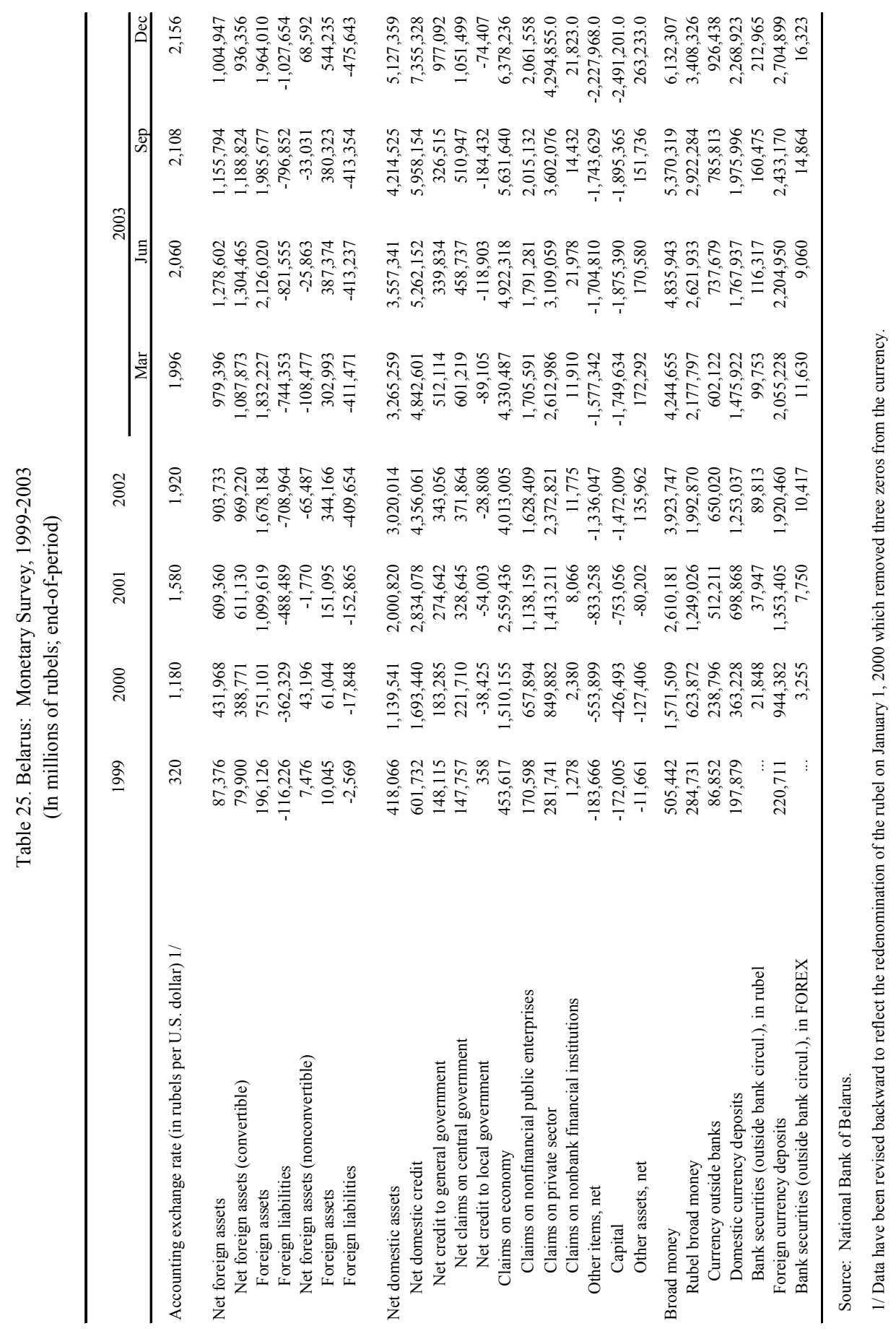




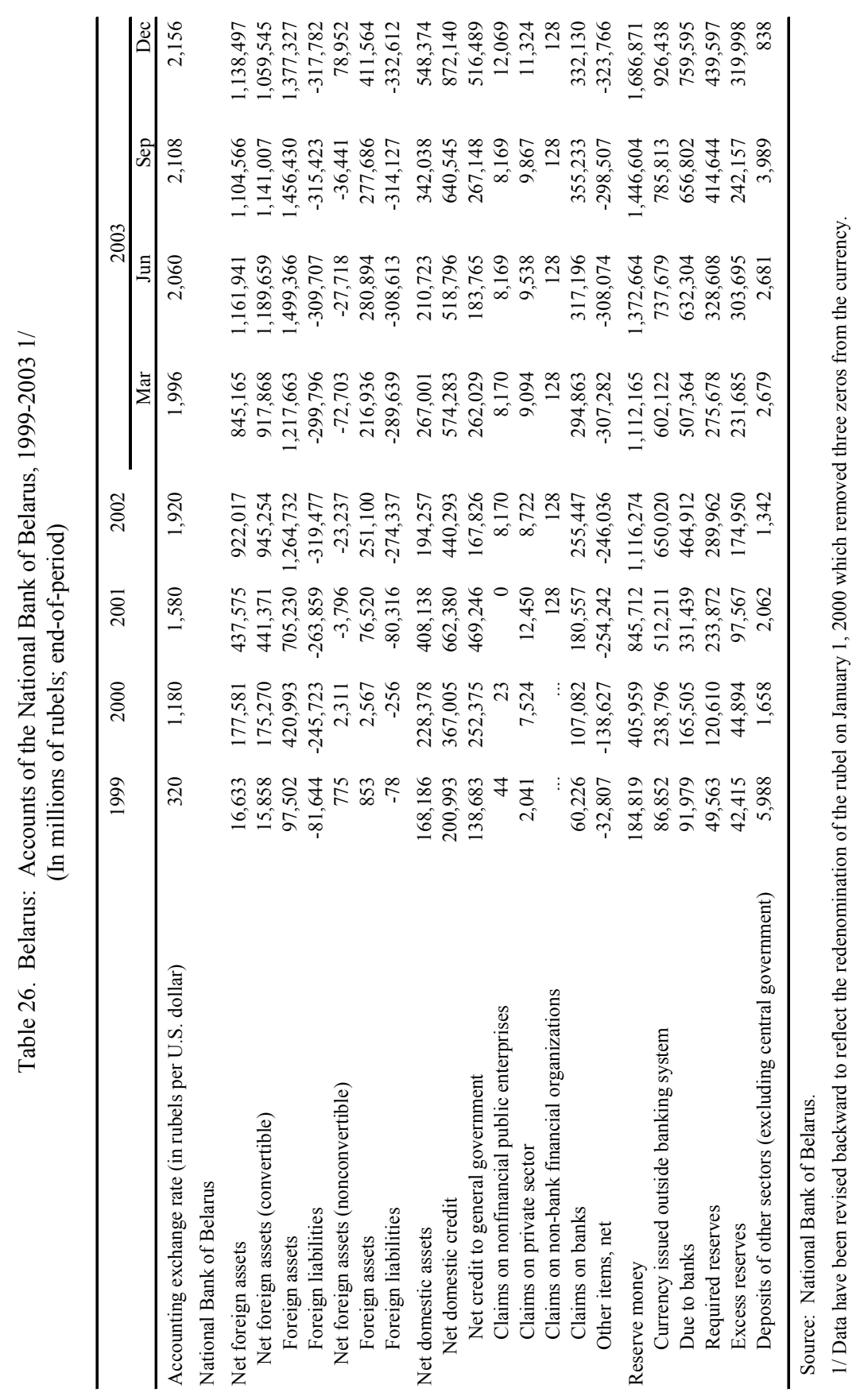




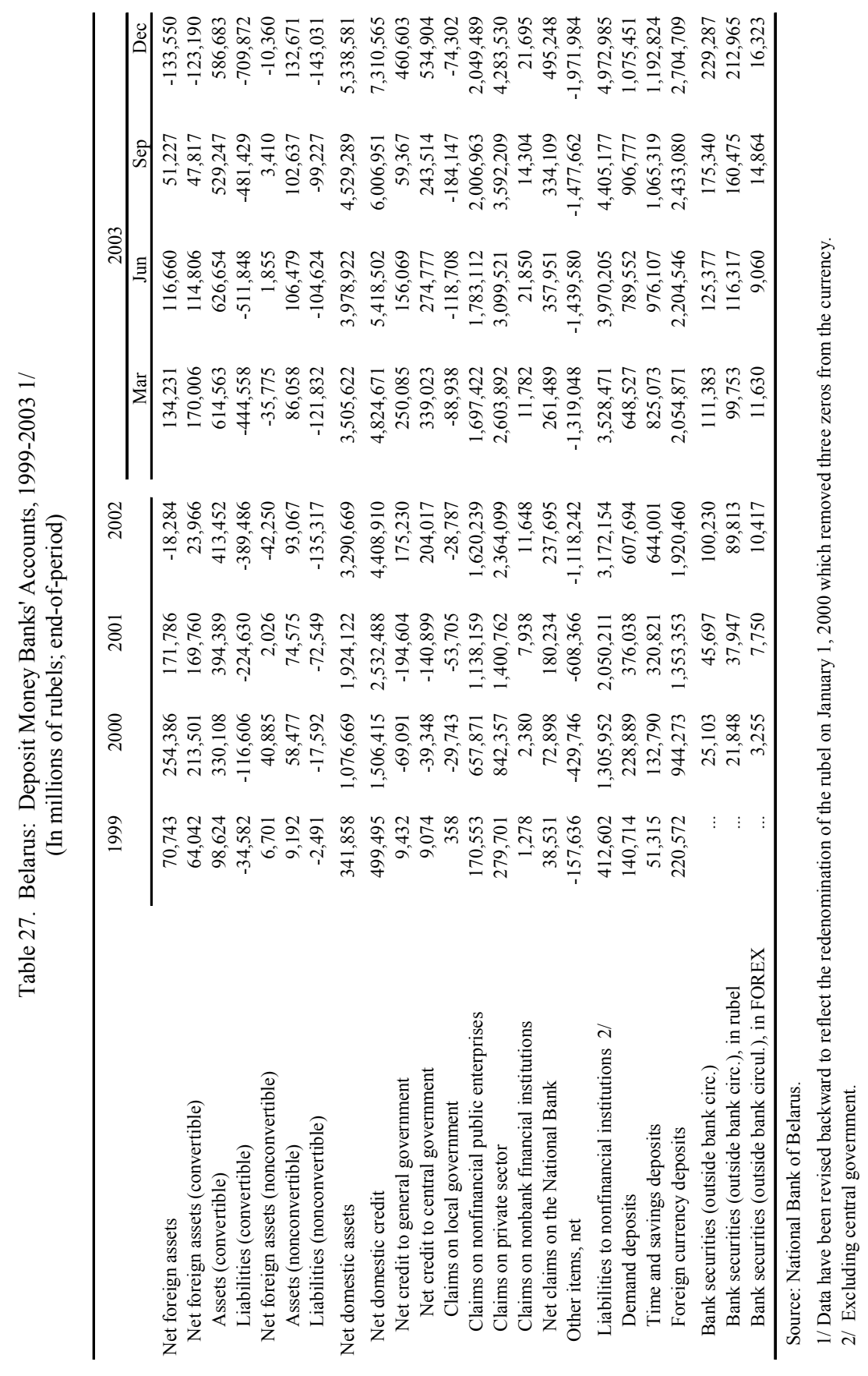


Table 28. Belarus: National Bank's Directed Credits, 1999-2003 1/ (In millions of rubels; end-of-period)

\begin{tabular}{|c|c|c|c|c|c|c|c|c|}
\hline & \multirow[t]{2}{*}{1999} & \multirow[t]{2}{*}{2000} & \multirow[t]{2}{*}{2001} & \multirow[t]{2}{*}{2002} & \multicolumn{4}{|c|}{2003} \\
\hline & & & & & Q1 & Q2 & Q3 & $\mathrm{Q} 4$ \\
\hline Directed credit & 27,695 & 26,525 & 15,942 & 30,422 & 31,405 & 32,394 & 32,371 & 32,354 \\
\hline Agriculture & 22 & 7 & 284 & $\ldots$ & $\ldots$ & $\ldots$ & $\ldots$ & $\ldots$ \\
\hline Trade & $\ldots$ & $\ldots$ & $\ldots$ & $\ldots$ & $\ldots$ & $\ldots$ & $\ldots$ & $\ldots$ \\
\hline Industry & 3,279 & 2,335 & 169 & $\ldots$ & $\ldots$ & $\ldots$ & $\ldots$ & $\ldots$ \\
\hline Housing & 24,383 & 23,981 & $\ldots$ & $\ldots$ & $\ldots$ & $\ldots$ & $\ldots$ & ... \\
\hline Other 2/ & 11 & 202 & 15,489 & 30,422 & 31,405 & 32,394 & 32,371 & 32,354 \\
\hline
\end{tabular}

Source: National Bank of Belarus.

1/ Data have been revised backward to reflect the redenomination of the rubel on January 1, 2000 which removed three zeros from the currency.

2/ NBB credits to Belarusbank for paying off deposits of Belbaltiya. 


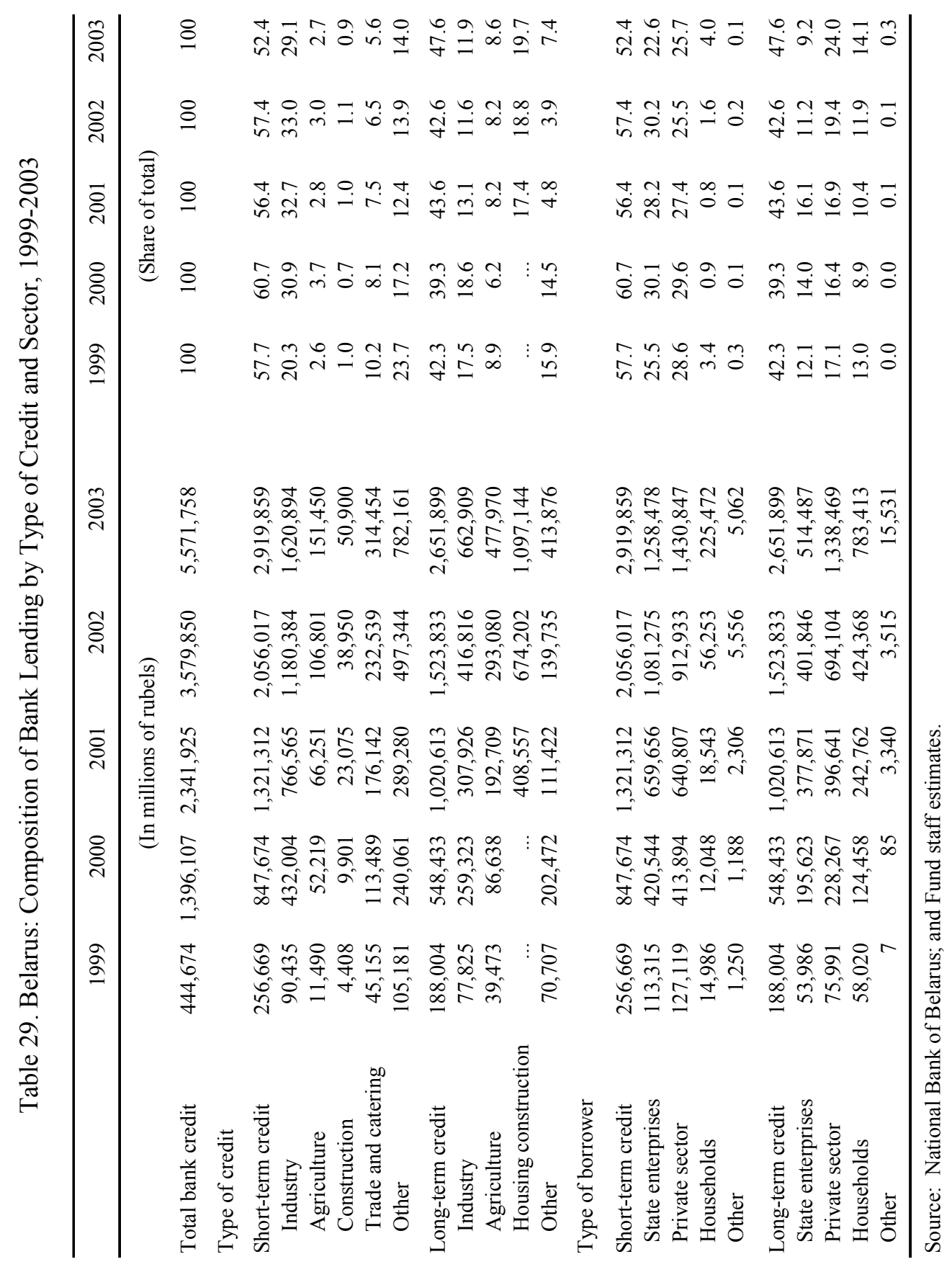


Table 30. Belarus: Auction of Securities Issued by the Ministry of Finance, 2001-03

(Weighted monthly averages)

\begin{tabular}{|c|c|c|c|c|c|c|c|c|c|c|c|c|}
\hline & \multicolumn{7}{|c|}{ Short-term securities } & \multicolumn{5}{|c|}{ Long-term securities } \\
\hline & $\begin{array}{c}\text { Maturity } \\
\text { (days) }\end{array}$ & $\begin{array}{c}\text { Amount } \\
\text { offered } \\
\text { (millions } \\
\text { of rubels) }\end{array}$ & $\begin{array}{c}\text { Amount } \\
\text { sold }\end{array}$ & $\begin{array}{c}\text { Weighted } \\
\text { average } \\
\text { price }\end{array}$ & $\begin{array}{l}\text { Annualized } \\
\text { yield } \\
\text { (percent) }\end{array}$ & $\begin{array}{l}\text { Monthly } \\
\text { average } \\
\text { yield } \\
\text { (percent) }\end{array}$ & $\begin{array}{l}\text { Number of } \\
\text { participants }\end{array}$ & $\begin{array}{l}\text { Maturity } \\
\text { (days) }\end{array}$ & $\begin{array}{c}\text { Amount } \\
\text { offered } \\
\text { (millions } \\
\text { of rubels) }\end{array}$ & $\begin{array}{c}\text { Amount } \\
\text { sold }\end{array}$ & $\begin{array}{c}\text { Weighted } \\
\text { average } \\
\text { price }\end{array}$ & $\begin{array}{l}\text { Number of } \\
\text { participants }\end{array}$ \\
\hline \multicolumn{13}{|l|}{2001} \\
\hline Jan & 101 & 55,935 & 47,338 & 83.5 & 77.3 & 6.4 & 3 & No auction & & & & \\
\hline Feb & 133 & 41,364 & 35,458 & 80.5 & 77.2 & 6.4 & 4 & No auction & & & & \\
\hline Mar & 166 & 60,820 & 41,139 & 83.3 & 73.2 & 6.1 & 6 & 730 & 3,300 & 3,300 & 100.0 & 1 \\
\hline Apr & 59 & 48,636 & 41,175 & 91.4 & 67.7 & 5.6 & 4 & 731 & 3,000 & 3,000 & 100.0 & 1 \\
\hline May & 86 & 38,142 & 36,797 & 88.4 & 65.1 & 5.4 & 6 & 730 & 3,000 & 3,000 & 100.0 & 1 \\
\hline Jun & 74 & 63,645 & 42,867 & 90.6 & 58.4 & 4.9 & 6 & 731 & 3,000 & 3,000 & 100.0 & 1 \\
\hline Jul & 38 & 72,862 & 63,152 & 95.2 & 50.5 & 4.2 & 7 & No auction & & & & \\
\hline Aug & 44 & 91,357 & 88,678 & 94.7 & 50.1 & 4.2 & 4 & 731 & 3,500 & 3,500 & 100.0 & 2 \\
\hline Sep & 26 & 60,202 & 58,733 & 96.7 & 49.4 & 4.1 & 8 & No auction & & & & \\
\hline Oct & 54 & 56,000 & 45,842 & 93.6 & 48.0 & 4.0 & 3 & 366 & 17,020 & 17,020 & 100.0 & 6 \\
\hline Nov & 102 & 24,000 & 36,544 & 88.7 & 48.0 & 4.0 & 4 & 468 & 3,000 & 3,000 & 100.0 & 1 \\
\hline Dec & 195 & 35,000 & 37,703 & 68.6 & 48.0 & 4.0 & 2 & No auction & & & & \\
\hline \multicolumn{13}{|l|}{2002} \\
\hline Jan & 133 & 16,000 & 7,721 & 82.9 & 57.0 & 4.8 & 3 & 366 & 132 & 132 & 100.0 & 1 \\
\hline Feb & 129 & 32,000 & 2586 & 81.1 & 66.0 & 5.5 & 4 & No auction & & & & \\
\hline Mar & 201 & 107,768 & 103,552 & 76.0 & 61.9 & 5.2 & 2 & 731 & 1,500 & 1,500 & 100.0 & 1 \\
\hline Apr & 181 & 79,411 & 54,639 & 79.6 & 55.9 & 4.7 & 3 & 730 & 1,500 & 1,500 & 100.0 & 1 \\
\hline May & 168 & 94,191 & 72,615 & 82.2 & 53.9 & 4.5 & 6 & 731 & 1,500 & 1,500 & 100.0 & 1 \\
\hline Jun & 114 & 37,930 & 37,770 & 87.8 & 49.2 & 4.1 & 2 & 732 & 1,500 & 1,500 & 100.0 & 1 \\
\hline Jul & 165 & 35,283 & 120,954 & 83.7 & 45.1 & 3.8 & 6 & 731 & 1,500 & 1,500 & 100.0 & 1 \\
\hline Aug & 188 & 24,000 & 31,215 & 82.6 & 42.7 & 3.6 & 4 & 732 & 1,500 & 1,500 & 100.0 & 1 \\
\hline Sep & 152 & 37,860 & 56,508 & 85.7 & 41.5 & 3.5 & 2 & 1,325 & 3,562 & 3,562 & 100.0 & 2 \\
\hline Oct & 249 & 81,237 & 63,483 & 79.4 & 39.4 & 3.3 & 7 & 731 & 1,500 & 1,500 & 100.0 & 1 \\
\hline Nov & 118 & 45,640 & 117,725 & 89.9 & 37.5 & 3.1 & 9 & 732 & 1,500 & 1,500 & 100.0 & 1 \\
\hline Dec & 260 & 14,000 & 15,715 & 78.7 & 38.0 & 3.2 & 2 & 731 & 1,500 & 1,500 & 100.0 & 1 \\
\hline \multicolumn{13}{|l|}{2003} \\
\hline Jan & 209 & 42,000 & 16,692 & 84.1 & 38.0 & 3.2 & 4 & 731 & 1,000 & 1,000 & 100.0 & 1 \\
\hline Feb & 254 & 59,828 & 46696 & 79.6 & 37.9 & 3.2 & 6 & No auction & & & & \\
\hline Mar & 304 & 122,509 & 95,577 & 72.7 & 37.6 & 3.1 & 6 & 641 & 11,300 & 10,088 & 100.0 & 1 \\
\hline Apr & 264 & 143,503 & 75,750 & 80.8 & 35.5 & 3.0 & 4 & 731 & 6,000 & 6,292 & 100.0 & 1 \\
\hline May & 237 & 88,884 & 71,701 & 88.3 & 34.3 & 2.9 & 7 & 731 & 3,000 & 3,000 & 100.0 & 1 \\
\hline Jun & 353 & 59,000 & 45,404 & 76.9 & 31.6 & 2.6 & 3 & 610 & 33,000 & 25,839 & 100.0 & 3 \\
\hline Jul & 358 & 29,000 & 22,594 & 77.9 & 29.0 & 2.4 & 6 & 941 & 71,700 & 65,305 & 100.0 & 2 \\
\hline Aug & 320 & 56,360 & 33,223 & 88.1 & 26.6 & 2.2 & 4 & 916 & 1,205 & 1,895 & 100.0 & 1 \\
\hline Sep & 358 & 40,000 & 10,316 & 79.0 & 27.1 & 2.3 & 4 & 565 & 3,174 & 710 & 100.0 & 1 \\
\hline Oct & 298 & 33,000 & 12,610 & 84.8 & 26.8 & 2.2 & 2 & No auction & & & & \\
\hline Nov & 173 & 65,349 & 60,337 & 91.8 & 26.3 & 2.2 & 4 & No auction & & & & \\
\hline Dec & 229 & 89,103 & 84,470 & 86.2 & 27.2 & 2.3 & 1 & 590 & 6,235 & 5,993 & 100.0 & 1 \\
\hline
\end{tabular}

Sources: National Bank of Belarus; and Fund staff estimates. 


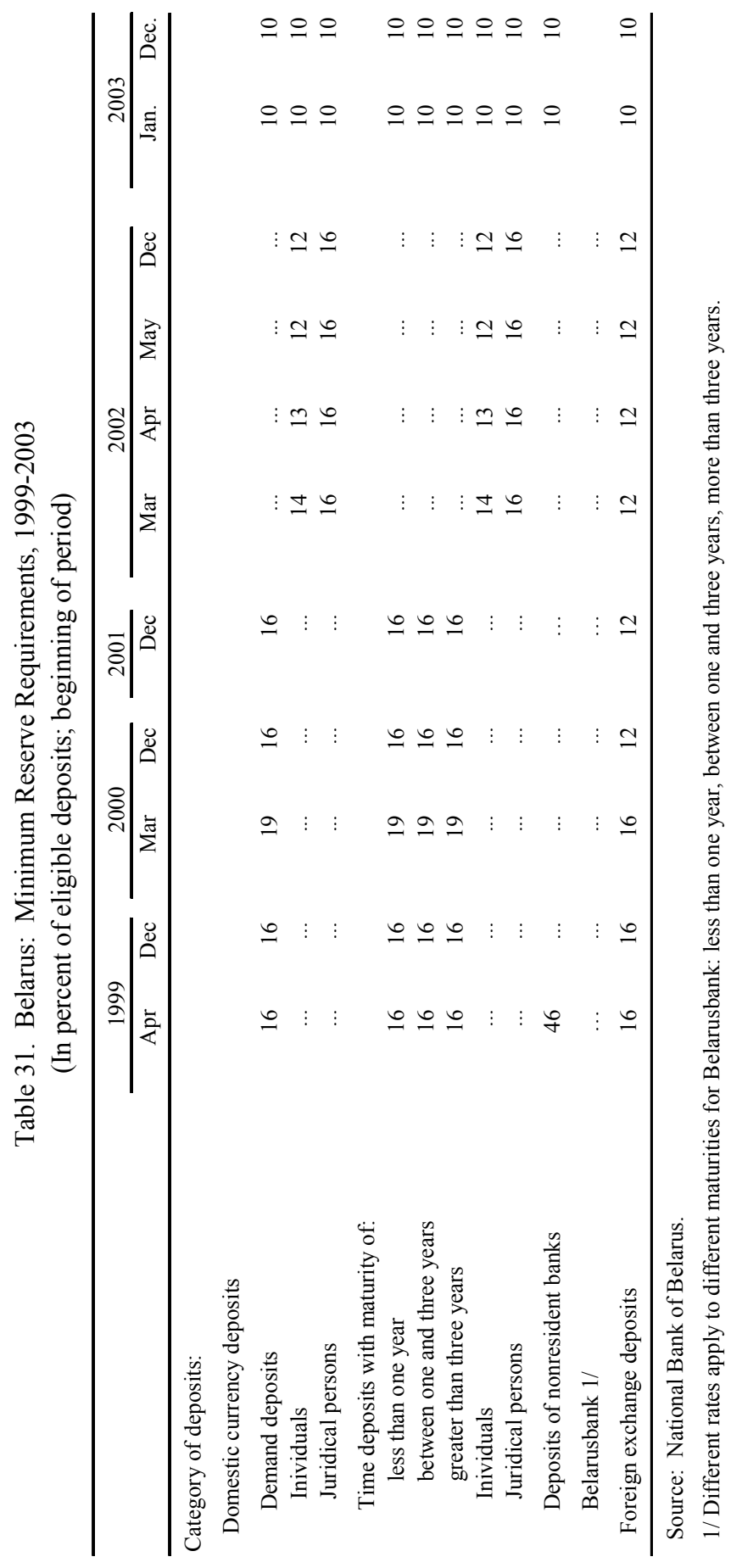


Table 32. Belarus: Interest Rates of the National Bank of Belarus, 1999-2003 (In percent per annum; beginning of period)

\begin{tabular}{|c|c|c|c|c|c|c|}
\hline & & \multicolumn{2}{|c|}{ Basic lombard rate } & \multicolumn{2}{|c|}{ Refinance rates } & \multirow{2}{*}{$\begin{array}{c}\text { Credit to } \\
\text { government 1/ }\end{array}$} \\
\hline & & $\begin{array}{r}\text { for period } \\
\text { of less than } \\
14 \text { days } \\
\end{array}$ & $\begin{array}{r}\text { for } 15 \text { to } \\
30 \text { days }\end{array}$ & Basic rate & $\begin{array}{r}\text { Average } \\
\text { refinance } \\
\text { rate } \\
\end{array}$ & \\
\hline 1999 & Dec & 120 & 125 & 110 & 22 & $0 / 6.5 / 110$ \\
\hline 2000 & Dec & 170 & 180 & 80 & 12 & $0 / 6.5 / 80$ \\
\hline 2001 & Dec & 105 & 115 & 48 & $\ldots$ & $0 / 6.5 / 48$ \\
\hline \multirow[t]{12}{*}{2002} & Jan & 105 & 115 & 48 & $\ldots$ & $0 / 6.5 / 48$ \\
\hline & Feb & 110 & 120 & 66 & $\ldots$ & $0 / 6.5 / 66$ \\
\hline & Mar & 110 & 120 & 66 & $\ldots$ & $0 / 6.5 / 66$ \\
\hline & Apr & 105 & 115 & 60 & $\ldots$ & $0 / 6.5 / 60$ \\
\hline & May & 95 & 105 & 55 & $\ldots$ & $0 / 6.5 / 55$ \\
\hline & Jun & 90 & 100 & 50 & $\ldots$ & $0 / 6.5 / 50$ \\
\hline & Jul & 85 & 95 & 46 & $\ldots$ & $0 / 6.5 / 46$ \\
\hline & Aug & 83 & 93 & 44 & $\ldots$ & $0 / 6.5 / 44$ \\
\hline & Sep & 78 & 88 & 42 & $\ldots$ & $0 / 6.5 / 42$ \\
\hline & Oct & 70 & 80 & 40 & $\ldots$ & $0 / 6.5 / 40$ \\
\hline & Nov & 65 & 73 & 39 & $\ldots$ & $0 / 6.5 / 39$ \\
\hline & Dec & 65 & 73 & 38 & $\ldots$ & $0 / 6.5 / 38$ \\
\hline \multirow[t]{12}{*}{2003} & Jan & 65 & 73 & 38 & $\ldots$ & $0 / 6.5$ \\
\hline & Feb & 65 & 73 & 38 & $\ldots$ & $0 / 6.5$ \\
\hline & Mar & 65 & 73 & 38 & $\ldots$ & $0 / 6.5$ \\
\hline & Apr & 65 & 73 & 37 & $\ldots$ & $0 / 6.5$ \\
\hline & May & 60 & 68 & 35 & $\ldots$ & $0 / 6.5$ \\
\hline & Jun & 55 & 60 & 34 & $\ldots$ & $0 / 6.5$ \\
\hline & Jul & 50 & $\ldots$ & 33 & $\ldots$ & $0 / 6.5$ \\
\hline & Aug & 48 & $\ldots$ & 31 & $\ldots$ & $0 / 6.5$ \\
\hline & Sep & 46 & $\ldots$ & 30 & $\ldots$ & $0 / 6.5$ \\
\hline & Oct & 46 & $\ldots$ & 29 & $\ldots$ & $0 / 6.5$ \\
\hline & Nov & 43 & $\ldots$ & 28 & $\ldots$ & $0 / 6.5$ \\
\hline & Dec & 40 & $\ldots$ & 28 & $\ldots$ & $0 / 6.5$ \\
\hline
\end{tabular}

Source: National Bank of Belarus.

1/ The interest rate on credit to the government differed according type of credit: 0 percent - on credits, financing the budgetary expenditure on compensation of losses of deposits of the population, budgetary directed credits to the agro-industrial complex; 6.5 percent - on deficit financing of the previous years; the refinance rate - on financing the budget deficit. 
Table 33. Belarus: Interest Rates on Rubel Bank Deposits, 2000-03 (In percent per annum)

\begin{tabular}{llllllll}
\hline & & Up to 1 & 2 to 3 months & 4 to 6 months & $\begin{array}{c}7 \text { to } 12 \\
\text { months }\end{array}$ & 2 to 3 years & $\begin{array}{c}\text { More than } 3 \\
\text { years }\end{array}$ \\
\hline 2000 & Dec & 90.5 & 96.3 & 100.7 & 106.6 & 110.1 & 116.3 \\
2001 & Dec & 49.5 & 60.4 & 63.5 & 66.2 & 72.5 & 47.9 \\
2002 & Jan & 53.4 & 63.6 & 65.8 & 67.3 & 70.9 & 84.5 \\
& Feb & 62.4 & 77.1 & 66.8 & 68.4 & 72.3 & 78.1 \\
Mar & 57.0 & 73.3 & 68.9 & 69.1 & 72.9 & 85.0 \\
Apr & 50.0 & 65.8 & 64.4 & 66.7 & 72.0 & 84.1 \\
May & 46.0 & 58.0 & 61.0 & 64.2 & 68.9 & 46.4 \\
Jun & 41.7 & 53.0 & 54.0 & 53.1 & 63.0 & 41.0 \\
Jul & 39.5 & 50.1 & 50.1 & 51.4 & 58.1 & 51.5 \\
Aug & 36.4 & 46.2 & 43.8 & 46.6 & 55.0 & 37.6 \\
Sep & 33.8 & 44.3 & 43.1 & 44.7 & 47.1 & 51.0 \\
Oct & 33.3 & 41.9 & 42.9 & 42.8 & 44.4 & 46.6 \\
Nov & 32.5 & 38.5 & 39.8 & 37.9 & 40.5 & 40.8 \\
Dec & 34.9 & 38.0 & 37.7 & 37.6 & 39.6 & 30.4 \\
Jan & 35.1 & 38.7 & 36.2 & 36.6 & 39.0 & 40.6 \\
Feb & 32.9 & 38.9 & 36.2 & 37.6 & 37.2 & 40.0 \\
Mar & 32.1 & 37.7 & 35.5 & 36.3 & 39.5 & 40.3 \\
Apr & 32.4 & 36.5 & 34.9 & 35.8 & 39.1 & 40.1 \\
May & 28.4 & 35.8 & 33.9 & 35.1 & 37.6 & 40.9 \\
Jun & 26.4 & 33.8 & 33.6 & 34.1 & 36.5 & 14.9 \\
Jul & 24.5 & 30.4 & 30.3 & 32.5 & 34.8 & 30.5 \\
Aug & 22.4 & 25.9 & 26.3 & 27.0 & 30.7 & 27.2 \\
Sep & 22.8 & 24.6 & 25.9 & 25.5 & 28.8 & 28.1 \\
Oct & 22.8 & 24.1 & 23.7 & 22.8 & 28.5 & 23.4 \\
Nov & 23.0 & 24.3 & 26.4 & 24.4 & 28.9 & 28.2 \\
Dec & 22.4 & 24.7 & 26.2 & 24.8 & 28.8 & 27.8 \\
\hline
\end{tabular}

Source: National Bank of Belarus. 
Table 34. Belarus: Interest Rates on Rubel Bank Credit, 2000-03

\begin{tabular}{|c|c|c|c|c|}
\hline & 2000 & 2001 & 2002 & 2003 \\
\hline $\begin{array}{l}\text { Average interest rates on all outstanding credits } \\
\text { to legal entities and individual entrepreneurs }\end{array}$ & 81.3 & 57.7 & 45.9 & 28.6 \\
\hline \multicolumn{5}{|l|}{ By form of ownership } \\
\hline Public & 84.9 & 63.0 & 50.6 & 30.7 \\
\hline Collective & 77.1 & $\ldots$ & $\ldots$ & $\ldots$ \\
\hline Private & 98.5 & 75.1 & 60.2 & 33.5 \\
\hline Other & 98.9 & 46.7 & 35.4 & 24.3 \\
\hline \multicolumn{5}{|l|}{ By type of business } \\
\hline Industry & 98.3 & 71.3 & 58.3 & 34.5 \\
\hline Agriculture & 25.7 & 17.0 & 13.3 & 10.7 \\
\hline Forestry & 82.4 & 57.0 & 45.5 & 29.9 \\
\hline Construction & 110.8 & 83.7 & 67.2 & 40.0 \\
\hline Trade and Catering & 97.4 & 73.6 & 60.8 & 39.7 \\
\hline Information services & 119.4 & 86.1 & 72.5 & 43.4 \\
\hline Real estate transactions & 120.2 & 94.5 & 63.9 & 41.2 \\
\hline Housing sector & 112.2 & 87.7 & 80.5 & 40.9 \\
\hline Communal and other services & 105.3 & 82.9 & 72.2 & 35.5 \\
\hline Other & 98.0 & 72.1 & 60.0 & 36.3 \\
\hline \multicolumn{5}{|l|}{ Other types of borrowers: } \\
\hline \multicolumn{5}{|l|}{ Households } \\
\hline consumer loans & 55.1 & 57.6 & 54.9 & 44.4 \\
\hline financing real estate & 6.6 & 7.0 & 7.7 & 8.5 \\
\hline Interbank credits & 107.1 & 80.1 & 45.3 & 26.0 \\
\hline Overdue credits & 130.4 & 96.0 & 67.8 & 35.0 \\
\hline $\begin{array}{l}\text { Average interest rates on new credits } \\
\text { to legal entities, physical persons, } \\
\text { and individual entrepreneurs }\end{array}$ & 929 & 70.4 & 58.5 & 368 \\
\hline \multicolumn{5}{|l|}{ By the term structure } \\
\hline up to 1 month & 99.7 & 76.2 & 63.1 & 40.3 \\
\hline from 1 to 3 months & 98.6 & 75.0 & 63.4 & 39.9 \\
\hline from 3 to 6 months & 103.1 & 75.4 & 59.3 & 38.4 \\
\hline from 6 to 12 months & 92.1 & 68.7 & 55.7 & 36.4 \\
\hline from 1 to 3 years & 81.2 & 69.8 & 50.4 & 34.0 \\
\hline more than 3 years & 12.9 & 13.1 & 12.1 & 13.5 \\
\hline
\end{tabular}

Source: National Bank of Belarus. 


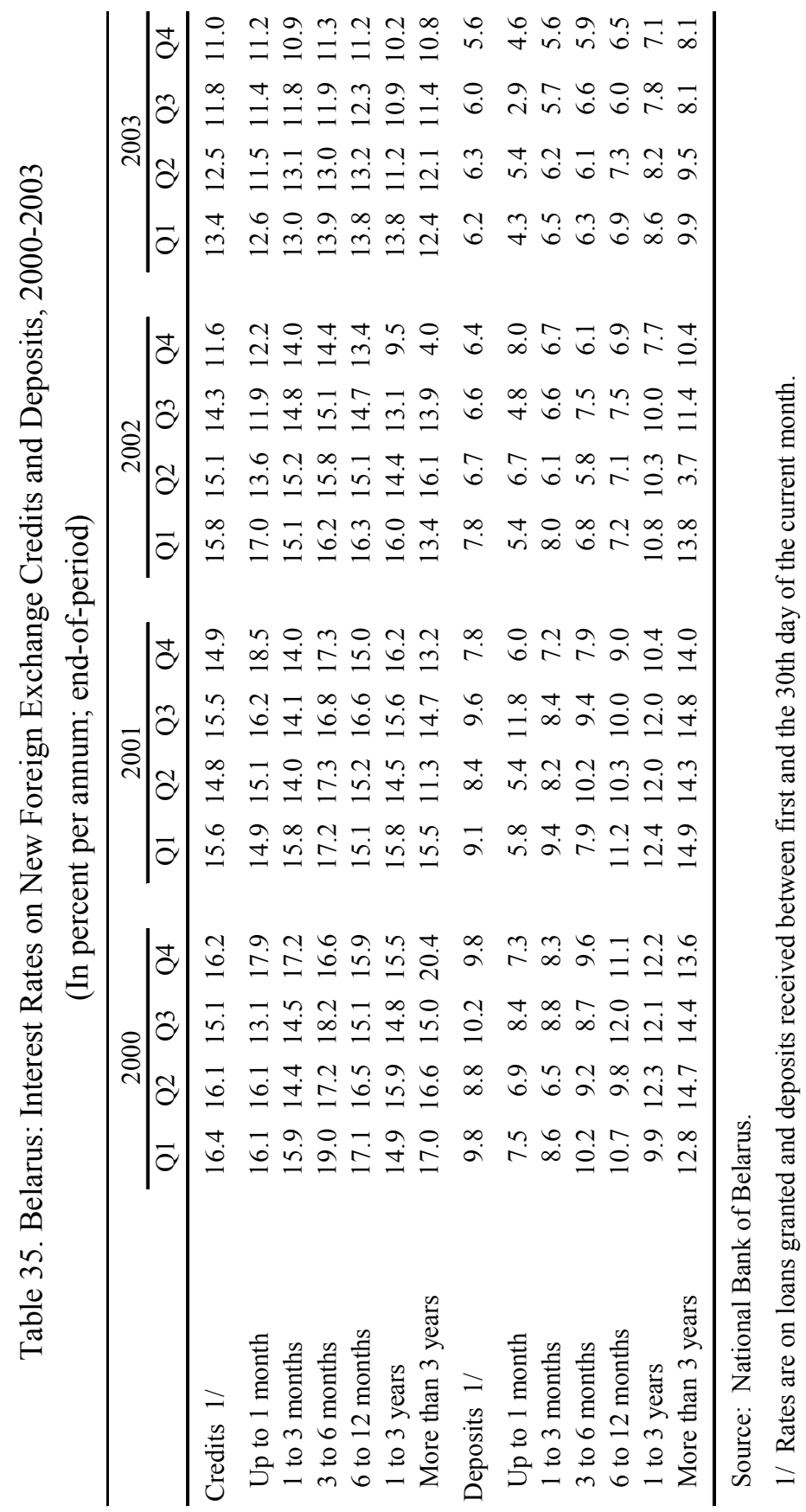


Table 36. Belarus: Structural Characteristics of the Banking Sector, 1999-2003 (In percent of total; end-of-period)

\begin{tabular}{|c|c|c|c|c|c|c|}
\hline & \multicolumn{5}{|c|}{ Former specialized banks } & \multirow{2}{*}{$\begin{array}{c}\text { Other } \\
\text { commercial } \\
\text { banks }\end{array}$} \\
\hline & Total & $\begin{array}{c}\text { Belagroprom- } \\
\text { bank }\end{array}$ & $\begin{array}{c}\text { Belpromstroi- } \\
\text { bank }\end{array}$ & $\begin{array}{l}\text { Belvneshekonom- } \\
\text { bank }\end{array}$ & $\begin{array}{c}\text { Belarus } \\
\text { bank }\end{array}$ & \\
\hline \multicolumn{7}{|c|}{ Paid-in capital } \\
\hline 1999 & 81.7 & 29.6 & 8.2 & 3.0 & 40.9 & 18.3 \\
\hline 2000 & 80.0 & 37.2 & 2.1 & 4.8 & 35.9 & 20.0 \\
\hline 2001 & 67.8 & 28.3 & 2.3 & 5.4 & 31.8 & 32.2 \\
\hline 2002 & 74.5 & 33.6 & 1.4 & 2.1 & 37.3 & 25.5 \\
\hline \multicolumn{7}{|c|}{ Domestic currency loans } \\
\hline 1999 & 81.8 & 29.5 & 9.6 & 1.9 & 40.8 & 18.2 \\
\hline 2000 & 82.7 & 29.9 & 7.9 & 3.0 & 41.9 & 17.3 \\
\hline 2001 & 85.6 & 30.2 & 5.8 & 2.2 & 47.4 & 14.4 \\
\hline 2002 & 88.1 & 29.5 & 6.5 & 1.7 & 50.4 & 11.9 \\
\hline 2003 & 88.8 & 29.5 & 6.0 & 1.3 & 52.0 & 11.2 \\
\hline \multicolumn{7}{|c|}{ Domestic currency deposits } \\
\hline 1999 & 68.9 & 11.3 & 30.6 & 2.9 & 24.1 & 31.1 \\
\hline 2000 & 65.7 & 14.7 & 21.3 & 2.9 & 26.8 & 34.3 \\
\hline 2001 & 73.3 & 5.7 & 8.9 & 8.9 & 49.8 & 26.7 \\
\hline 2002 & 72.3 & 13.3 & 11.2 & 3.2 & 44.6 & 27.7 \\
\hline 2003 & 70.1 & 11.1 & 11.2 & 3.2 & 44.6 & 29.9 \\
\hline \multicolumn{7}{|c|}{ Refinancing from NBB } \\
\hline 1999 & 90.7 & 40.6 & 0.9 & 0.1 & 49.1 & 9.3 \\
\hline 2000 & 89.2 & 42.9 & 1.5 & 0.0 & 44.8 & 10.8 \\
\hline 2001 & 71.1 & 39.0 & 0.3 & 0.0 & 31.7 & 28.9 \\
\hline 2002 & 100.0 & 9.0 & 0.0 & 0.0 & 91.0 & 0.0 \\
\hline
\end{tabular}

Source: National Bank of Belarus. 
Table 37. Belarus: Selected Banking Indicators, 1999-2003 (In millions of rubels, unless otherwise indicated; end-of-period)

\begin{tabular}{|c|c|c|c|c|c|}
\hline & 1999 & 2000 & 2001 & 2002 & 2003 \\
\hline Capital fund & 133,434 & 387,572 & 592,022 & $1,212,808$ & $2,147,209$ \\
\hline Authorized funds & 99,767 & 210,321 & 317,146 & 815,954 & $1,446,562$ \\
\hline Unrealized exchange rate gains & 19,157 & 77,138 & 88,914 & 93,522 & 104,059 \\
\hline Retained profits & 4,852 & 22,100 & 20,184 & 40,158 & 111,004 \\
\hline Reevaluation fund & 3,574 & 67,872 & 191,783 & 233,005 & 316,830 \\
\hline Main funds amortization & 3,736 & 29,788 & 80,588 & 117,032 & 177,775 \\
\hline Long-term capital investments & 59 & 9,920 & 3,422 & 6,421 & 4,628 \\
\hline Nonmaterial assets & 7 & 2,409 & 5,712 & 3,107 & 1,890 \\
\hline Shortfall in provisions against unrealized losses & 11,946 & 35,317 & 105,771 & 176,880 & 112,917 \\
\hline Shares purchased by banks & 89 & 127 & 1,702 & 10,398 & 10,770 \\
\hline \multicolumn{6}{|l|}{ Assets } \\
\hline Balance sheet assets & 800,992 & $2,507,459$ & $6,969,266$ & $10,975,929$ & $16,882,205$ \\
\hline Risk weighted assets & 423,876 & $1,586,624$ & $2,857,337$ & $4,547,798$ & $7,084,499$ \\
\hline (In percent of balance sheet assets) & 52.9 & 63.3 & 41.0 & 41.4 & 42.0 \\
\hline Capital adequacy ratio $1 /$ & 31.5 & 24.4 & 20.7 & 24.2 & 27.3 \\
\hline Gross credit & 524,344 & $1,701,805$ & $2,726,595$ & $4,286,480$ & $6,959,088$ \\
\hline (In percent of assets) & 65.5 & 67.9 & 39.1 & 39.1 & 41.2 \\
\hline Arrears to banks & 53,194 & 199,583 & 332,648 & 299,869 & 231,396 \\
\hline (In percent of gross credit) & 10.1 & 11.7 & 12.2 & 7.0 & 3.3 \\
\hline (In percent of capital fund) & 39.9 & 51.5 & 56.2 & 24.7 & 10.8 \\
\hline Principal arrears & 49,437 & 186,167 & 317,006 & 291,316 & 224,110 \\
\hline (In percent of gross credit) & 9.4 & 10.9 & 11.6 & 6.8 & 3.2 \\
\hline Interest arrears & 3,756 & 13,416 & 15,642 & 8,553 & 7,286 \\
\hline (In percent of gross credit) & 0.7 & 0.8 & 0.6 & 0.2 & 0.1 \\
\hline Required provisions against bad loans & 42,563 & 157,611 & 248,853 & 248,301 & 233,080 \\
\hline (In percent of total loans) & 8.1 & 9.2 & 9.1 & 5.8 & 3.3 \\
\hline (In percent of capital fund) & 31.9 & 40.7 & 42.0 & 20.5 & 10.9 \\
\hline Actual provisions against bad loans & 30,618 & 122,294 & 143,082 & 71,422 & 120,163 \\
\hline (In percent of total loans) & 5.8 & 7.1 & 5.2 & 1.7 & 1.7 \\
\hline (In percent of capital fund) & 22.9 & 31.6 & 24.2 & 5.9 & 5.6 \\
\hline Profits & 9,224 & 18,520 & 28,724 & 53,710 & 131,469 \\
\hline (In percent of gross credit) & 1.8 & 1.1 & 1.0 & 1.3 & 1.9 \\
\hline \multicolumn{6}{|l|}{ Memorandum items: } \\
\hline \multicolumn{6}{|l|}{ Liquidity ratio } \\
\hline Requirement & 1.0 & 1.0 & 1.0 & 1.0 & 1.2 \\
\hline Unweighted average & 1.5 & 2.9 & 11.8 & 6.2 & 1.9 \\
\hline
\end{tabular}

Source: National Bank of Belarus.

1/ Adjusted for off-balance liabilities of banks in 2002-03. 
Table 38. Belarus: Six Largest Commercial Banks, Selected Indicators, 1999-2003 1/

(In millions of rubels, unless otherwise indicated; end-of-period)

\begin{tabular}{|c|c|c|c|c|c|}
\hline & 1999 & 2000 & 2001 & 2002 & 2003 \\
\hline $\begin{array}{l}\text { Capital fund } \\
\text { (In percent of all banks' capital fund) }\end{array}$ & $\begin{array}{r}108,416 \\
81.3\end{array}$ & $\begin{array}{r}275,961 \\
71.2\end{array}$ & $\begin{array}{r}328,786 \\
75.5\end{array}$ & $\begin{array}{r}764,637 \\
79.4\end{array}$ & $\begin{array}{r}1,794,430.6 \\
83.6\end{array}$ \\
\hline \multicolumn{6}{|l|}{ Assets } \\
\hline $\begin{array}{l}\text { Balance sheet assets } \\
\text { (In percent of all banks' assets) }\end{array}$ & $\begin{array}{r}667,351 \\
83.3\end{array}$ & $\begin{array}{r}2,066,143 \\
82.4\end{array}$ & $\begin{array}{r}6,212,265 \\
89.1\end{array}$ & $\begin{array}{r}6,982,793 \\
88.4\end{array}$ & $\begin{array}{r}14,914,266.2 \\
88.3\end{array}$ \\
\hline $\begin{array}{l}\text { Risk-weighted assets } \\
\text { (In percent of all banks' assets) } \\
\text { (In percent of balance sheet assets) }\end{array}$ & $\begin{array}{r}344,803 \\
81.3 \\
51.7\end{array}$ & $\begin{array}{r}1,302,231 \\
82.1 \\
63.0\end{array}$ & $\begin{array}{r}2,454,343 \\
85.9 \\
39.5\end{array}$ & $\begin{array}{r}3,872,270 \\
85.1 \\
55.5\end{array}$ & $\begin{array}{r}6,049,474.2 \\
85.4 \\
40.6\end{array}$ \\
\hline Capital adequacy ratio 2/ & 0.3 & 21.2 & 16.4 & 22.4 & 26.7 \\
\hline $\begin{array}{l}\text { Gross credit } \\
\text { (In percent of total banks' gross credit) } \\
\text { (In percent of assets) }\end{array}$ & $\begin{array}{r}469,125 \\
89.5 \\
70.3\end{array}$ & $\begin{array}{r}1,481,312 \\
87.0 \\
71.7\end{array}$ & $\begin{array}{r}2,419,702 \\
88.7 \\
39.0\end{array}$ & $\begin{array}{r}3,727,232 \\
87.0 \\
53.4\end{array}$ & $\begin{array}{r}6,071,552.8 \\
87.2 \\
40.7\end{array}$ \\
\hline $\begin{array}{l}\text { Arrears to banks } \\
\text { (In percent of total arrears) } \\
\text { (In percent of gross credit) } \\
\text { (In percent of capital fund) }\end{array}$ & $\begin{array}{r}47,498 \\
89.3 \\
10.1 \\
43.8\end{array}$ & $\begin{array}{r}172,943 \\
86.7 \\
11.7 \\
62.7\end{array}$ & $\begin{array}{r}3,129 \\
65.3 \\
0.1 \\
1.0\end{array}$ & $\begin{array}{r}493 \\
91.7 \\
0.0 \\
0.1\end{array}$ & $\begin{array}{r}218,536.1 \\
94.4 \\
3.6 \\
12.2\end{array}$ \\
\hline $\begin{array}{l}\text { Principal arrears } \\
\text { (In percent of gross credit) }\end{array}$ & $\begin{array}{r}43,914 \\
9.4\end{array}$ & $\begin{array}{r}160,316 \\
10.8\end{array}$ & $\begin{array}{r}63,330 \\
2.6\end{array}$ & $\begin{array}{r}71,793 \\
1.9\end{array}$ & $\begin{array}{r}211,892.1 \\
3.5\end{array}$ \\
\hline $\begin{array}{l}\text { Interest arrears } \\
\text { (In percent of gross credit) }\end{array}$ & $\begin{array}{r}3,584 \\
0.8\end{array}$ & $\begin{array}{r}12,627 \\
0.9\end{array}$ & $\begin{array}{r}15,642 \\
0.6\end{array}$ & $\begin{array}{r}8,553 \\
0.2\end{array}$ & $\begin{array}{r}6,644.0 \\
0.1\end{array}$ \\
\hline $\begin{array}{l}\text { Required provisions against bad loans } \\
\text { (In percent of total loans) } \\
\text { (In percent of capital fund) }\end{array}$ & $\begin{array}{r}37,175 \\
7.9 \\
34.3\end{array}$ & $\begin{array}{r}132,946 \\
8.9 \\
48.2\end{array}$ & $\begin{array}{r}248,853 \\
7.5 \\
75.7\end{array}$ & $\begin{array}{r}248,301 \\
5.1 \\
32.5\end{array}$ & $\begin{array}{r}213,705.6 \\
3.5 \\
11.9\end{array}$ \\
\hline $\begin{array}{l}\text { Actual provisions against bad loans } \\
\text { (In percent of total loans) } \\
\text { (In percent of capital fund) }\end{array}$ & $\begin{array}{r}28,140 \\
6.0 \\
26.0\end{array}$ & $\begin{array}{r}106,413 \\
7.1 \\
38.6\end{array}$ & $\begin{array}{r}143,082 \\
4.3 \\
43.5\end{array}$ & $\begin{array}{r}71,422 \\
1.5 \\
9.3\end{array}$ & $\begin{array}{r}110,675.2 \\
1.8 \\
6.2\end{array}$ \\
\hline $\begin{array}{l}\text { Profits } \\
\text { (In percent of total profits) } \\
\text { (In percent of gross credit) }\end{array}$ & $\begin{array}{r}6,879 \\
74.6 \\
1.5\end{array}$ & $\begin{array}{r}27,057 \\
79.0 \\
1.7\end{array}$ & $\begin{array}{r}18,530 \\
64.5 \\
0.8\end{array}$ & $\begin{array}{r}39,863 \\
74.2 \\
1.1\end{array}$ & $\begin{array}{r}106,928.6 \\
81.3 \\
1.8\end{array}$ \\
\hline \multicolumn{6}{|l|}{ Memorandum item: } \\
\hline
\end{tabular}

Source: National Bank of Belarus.

1/ Includes Belarusbank, Promstroibank, Agroprombank, Businessbank, Priorbank, and Vnesheconombank.

2/ Adjusted for off-balance liabilities of banks in 2001-03 
Table 39. Belarus: Commercial Banks and Branches of Foreign Banks (As of January 1, 2003)

\begin{tabular}{rlrrr}
\hline & & $\begin{array}{c}\text { Date of } \\
\text { establishment }\end{array}$ & $\begin{array}{c}\text { Number } \\
\text { of branches }\end{array}$ & $\begin{array}{c}\text { Total assets } \\
\text { (in millions of rubels) }\end{array}$ \\
\hline 1 & Belagroprombank 1/ & Sep 3, 1991 & 132 & $1,416,223$ \\
2 & Belpromstroibank 1/ & Dec 28, 1991 & 56 & 827,968 \\
3 & Belarusbank 1/ & Oct 27, 1995 & 154 & $5,049,987$ \\
4 & Belinvestbank & Sep 3, 2001 & 53 & 750,209 \\
5 & Priorbank & Jul 12, 1991 & 20 & 998,851 \\
6 & Belvneshekonombank 1/ & Dec 12, 1991 & 28 & 719,698 \\
7 & Poisk & May 15, 1991 & 2 & 26,709 \\
8 & Belnarodni & Apr 16, 1992 & $\ldots$ & 25,324 \\
9 & Belarus Industrial Bank & Oct 30,1991 & 5 & 40,808 \\
10 & Belgazprombank & Aug 19, 1991 & 7 & 141,119 \\
11 & Absolutbank & Dec 29,1993 & 2 & 19,908 \\
12 & Gem-Bank & Aug 26, 1991 & $\ldots$ & 52,558 \\
13 & Minsk Kompleksbank & Feb 21, 1994 & $\ldots$ & 102,633 \\
14 & Bank Reconverzi i Razvitiya & Feb 22, 1994 & 2 & 31,139 \\
15 & Minsk Tranzitnibank & Mar 14, 1994 & 5 & 46,041 \\
16 & Trade-industry Bank & April 1, 1994 & $\ldots$ & 16,720 \\
17 & Tekhnobank & Aug 5, 1994 & 2 & 63,573 \\
18 & Zolotoi Taler & Oct 5, 1994 & 2 & 49,914 \\
19 & Infobank & Nov 9, 1994 & 4 & 93,651 \\
20 & Slavneftebank & Oct 7, 1996 & 5 & 173,663 \\
21 & Mezhtorgbank & Jan. 28, 1999 & 1 & 215,186 \\
22 & Moscow-Minsk & Apr. 7, 2000 & $\ldots$ & 65,684 \\
23 & Astanaeksimbank & Jul 25, 2002 & $\ldots$ & 14,199 \\
24 & UBS Bank & Oct 7, 2002 & $\ldots$ & 11,175 \\
25 & LoroBank & Jul 10, 2002 & $\ldots$ & 1,245 \\
26 & Atom-Bank & Jan. 25, 2001 & $\ldots$ & 17,552 \\
27 & Northerns Investment Bank & Sep 27, 2001 & $\ldots$ & 2,649 \\
28 & International Reserve Bank & Feb 25, 2002 & $\ldots$ & 1,544 \\
\hline & & & & \\
\hline
\end{tabular}

Sources: National Bank of Belarus; and Fund staff estimates.

1/ Former specialized bank. 
Table 40. Belarus: Official Exchange Rates, 1999-2003 1/

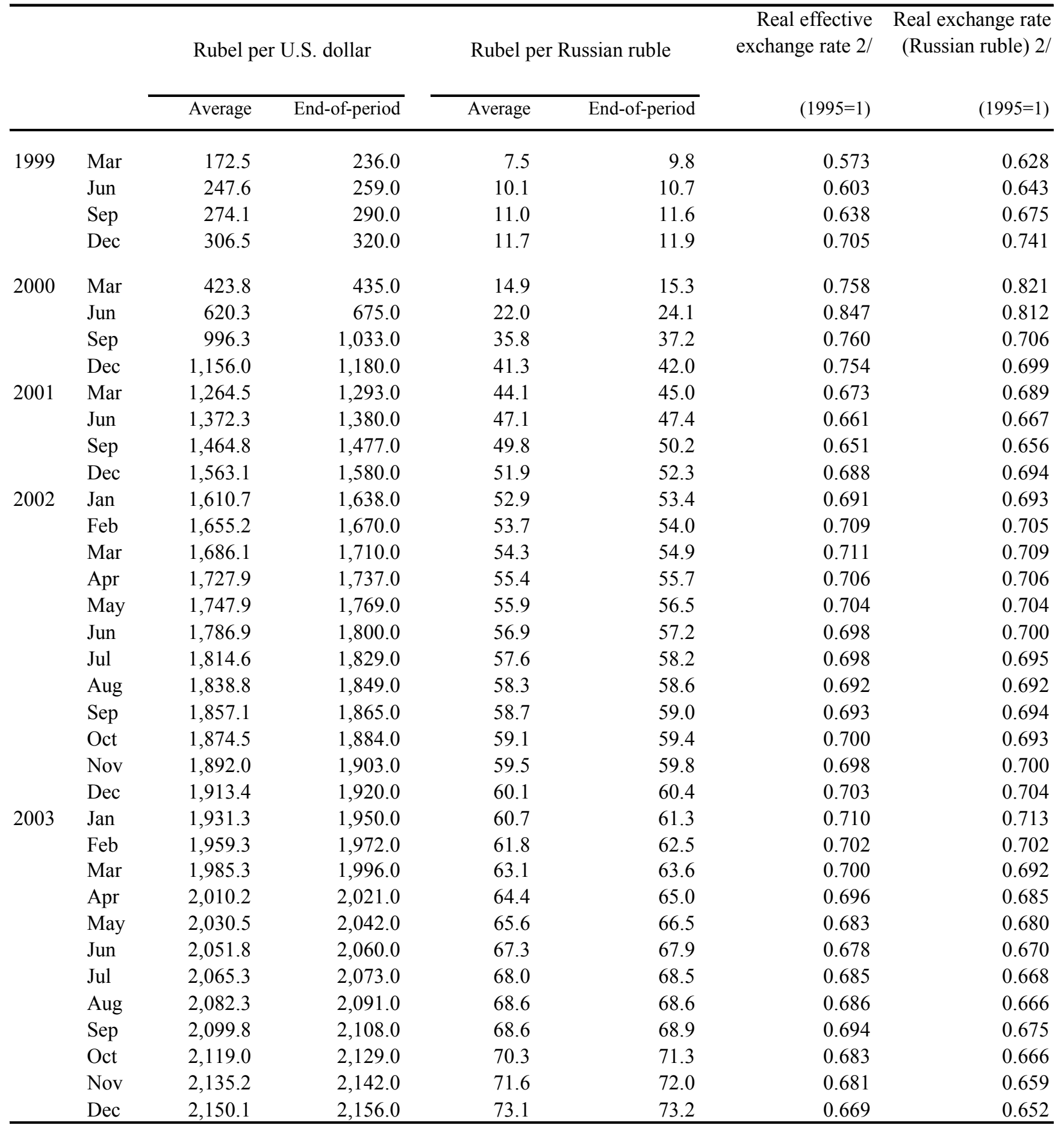


Table 41. Belarus: Direction of Trade: Exports and Imports, 1999-2003 (In millions of U.S. dollars, unless otherwise indicated)

\begin{tabular}{lrrrrr}
\hline & 1999 & 2000 & 2001 & 2002 & 2003 \\
\hline Total exports & 5,909 & 7,326 & 7,451 & 8,021 & 9,964 \\
CIS exports & 3,622 & 4,399 & 4,494 & 4,384 & 5,453 \\
Of which: & & & & & \\
$\quad$ Russia & 3,222 & 3,710 & 3,963 & 3,977 & 4,899 \\
$\quad$ Ukraine & 281 & 560 & 422 & 272 & 344 \\
$\quad$ Kazakhstan & 28 & 20 & 29 & 39 & 68 \\
$\quad$ Other countries & 91 & 109 & 80 & 96 & 142 \\
Non-CIS exports & 2,287 & 2,927 & 2,957 & 3,637 & 4,511 \\
$\quad$ Germany & 215 & 232 & 241 & 348 & 421 \\
$\quad$ Poland & 208 & 277 & 248 & 273 & 434 \\
$\quad$ Other countries & 1,864 & 2,418 & 2,468 & 3,016 & 3,656 \\
Total imports & 6,674 & 8,646 & 8,286 & 9,092 & 11,505 \\
CIS imports & 4,289 & 6,070 & 5,797 & 6,295 & 8,006 \\
Of which: & & & & & \\
$\quad$ Russia & 3,767 & 5,605 & 5,438 & 5,922 & 7,559 \\
$\quad$ Ukraine & 416 & 341 & 277 & 1 & 362 \\
$\quad$ Kazakhstan & 12 & 45 & 8 & 15 & 14 \\
$\quad$ Other countries & 94 & 79 & 74 & 67 & 71 \\
Non-CIS imports & 2,385 & 2,576 & 2,489 & 2,797 & 3,499 \\
$\quad$ Germany & 693 & 588 & 604 & 693 & 820 \\
$\quad$ Poland & 212 & 224 & 202 & 222 & 348 \\
$\quad$ Other countries & 1,480 & 1,764 & 1,683 & 1,882 & 2,331 \\
Memorandum items: & & & & & \\
Share of CIS exports to total exports & 61 & 60 & 60 & 55 & 55 \\
$\quad$ Russia & 55 & 51 & 53 & 50 & 49 \\
Share of CIS imports to total imports & 64 & 70 & 70 & 69 & 70 \\
$\quad$ Russia & 56 & 65 & 66 & 65 & 66 \\
\hline Sourc: Misty of Stistics & & & & &
\end{tabular}

Source: Ministry of Statistics and Analysis. 
Table 42. Belarus: Balance of Payments, 1999-2003

(In millions of U.S. dollars, unless otherwise indicated)

\begin{tabular}{|c|c|c|c|c|c|}
\hline & 1999 & 2000 & 2001 & 2002 & $\begin{array}{r}2003 \\
\text { Jan-Sep }\end{array}$ \\
\hline Current account balance & -193.7 & -338.4 & -434.9 & -377.5 & -291.5 \\
\hline Merchandise trade balance & -570.0 & -884.1 & -806.7 & -914.3 & -699.5 \\
\hline Exports & $5,646.4$ & $6,640.5$ & $7,334.1$ & $7,964.7$ & $7,308.9$ \\
\hline Imports & $-6,216.4$ & $-7,524.6$ & $-8,140.8$ & $-8,879.0$ & $-8,008.4$ \\
\hline Services (net) & 314.5 & 437.7 & 260.5 & 391.8 & 328.0 \\
\hline Income (net) & -42.0 & -46.7 & -42.8 & -28.6 & -20.1 \\
\hline Transfers (net) & 103.8 & 154.7 & 154.1 & 173.6 & 100.1 \\
\hline Capital and financial accounts & 454.1 & 209.5 & 321.3 & 535.2 & 211.9 \\
\hline Capital account & 54.6 & 69.4 & 56.3 & 52.7 & 47.8 \\
\hline Financial account & 399.5 & 140.1 & 265.0 & 482.5 & 164.1 \\
\hline Direct investment (net) & 443.2 & 118.6 & 95.5 & 453.3 & 89.9 \\
\hline Portfolio investment (net) & -20.6 & 44.4 & -19.9 & -9.1 & 16.0 \\
\hline Trade Credits (net) & -13.8 & -69.7 & -55.6 & -65.1 & 125.9 \\
\hline Loans (net) & 24.2 & 107.8 & 261.9 & 221.1 & -11.9 \\
\hline Other (net) & -33.5 & -61.0 & -16.9 & -117.7 & -55.8 \\
\hline Errors and omissions & -240.5 & 254.1 & 35.1 & -60.7 & 171.4 \\
\hline Overall balance & 19.9 & 125.2 & -78.5 & 97.0 & 91.8 \\
\hline Financing & -19.9 & -125.2 & 78.5 & -97.0 & -91.8 \\
\hline Gross official reserves & 34.5 & -75.6 & 5.2 & -100.9 & 5.7 \\
\hline Use of Fund resources & -58.0 & -55.8 & -29.8 & -30.0 & -32.0 \\
\hline Short-term loans & $\ldots$ & $\ldots$ & 68.4 & 88.7 & 15.1 \\
\hline O/w: Central Bank of Russia 1/ & & $\ldots$ & 51.6 & 94.9 & \\
\hline Exceptional financing 2/ & 3.6 & 6.2 & 34.7 & -54.8 & -80.6 \\
\hline \multicolumn{6}{|l|}{ Memorandum items: } \\
\hline Current account (as percent of GDP) & -1.6 & -2.6 & -3.5 & -2.6 & -2.3 \\
\hline Trade balance (as percent of GDP) & -4.7 & -6.8 & -6.5 & -6.3 & -5.5 \\
\hline Overall balance (as percent of GDP) & 0.2 & 1.0 & -0.6 & 0.7 & 0.7 \\
\hline Y-o-y growth in exports of goods (in percent) & -8.5 & 17.6 & 10.4 & 8.6 & 27.5 \\
\hline Y-o-y growth in imports of goods (in percent) & -19.0 & 21.0 & 8.2 & 9.1 & 29.7 \\
\hline Gross official reserves & 309.0 & 356.8 & 359.4 & 456.6 & 459.2 \\
\hline In months of imports of goods and services & 0.6 & 0.5 & 0.5 & 0.6 & 0.5 \\
\hline Medium and long-term debt & 668.1 & 673.6 & 816.7 & 991.4 & $1,083.1$ \\
\hline (as percent of GDP) & 5.5 & 5.1 & 6.6 & 6.8 & \\
\hline Short-term debt (exc. portfolio) & 1556.5 & $1,448.9$ & $1,630.3$ & $2,086.9$ & $2,167.1$ \\
\hline (as percent of GDP) & 12.9 & 11.1 & 13.2 & 14.3 & \\
\hline Debt service ratio (as percent of exports of goods and services) & 3.9 & 4.2 & 3.4 & 4.1 & .. \\
\hline \multicolumn{6}{|l|}{ Public and public-guaranteed debt service ratio } \\
\hline (as percent of exports of goods and services) & 2.0 & 1.7 & 1.3 & 1.6 & 4.8 \\
\hline External arrears 3/ & 447.6 & 453.8 & 488.5 & 433.7 & 353.1 \\
\hline In percent of GDP & 3.7 & 3.5 & 4.0 & 3.0 & \\
\hline
\end{tabular}

Sources: Belarus authorities and IMF staff estimates.

1/ Stabilization loan from Russia in preparation for monetary union. The loan is assumed to be repaid by end-2004.

2/ Includes accumulation, repayment, and forgiveness of arrears.

3/ As of end-2002, external arrears included US\$214 million for gas, US\$12.8 million for oil and oil products, US\$54 million for electricity, US\$152.6 million for the other goods, and \$0.5 million on public and public guaranteed debt. 
Table 43. Belarus: Selected International Liabilities, 1999-2003

(In millions of U.S. dollars, unless otherwise indicated; end-of-period)

\begin{tabular}{|c|c|c|c|c|c|}
\hline & 1999 & 2000 & 2001 & 2002 & 2003 \\
\hline \multicolumn{6}{|l|}{$\begin{array}{l}\text { Total medium- and long-term public and publicly- } \\
\text { guaranteed external debt }\end{array}$} \\
\hline Multilateral (including IMF) & 449.0 & 340.4 & 285.9 & 235.3 & 176.1 \\
\hline IMF & 178.0 & 112.1 & 81.1 & 55.1 & 24.0 \\
\hline World Bank & 130.0 & 121.1 & 114.7 & 103.7 & 89.9 \\
\hline EBRD & 111.0 & 81.3 & 66.6 & 57.8 & 47.2 \\
\hline European Union & 30.0 & 25.9 & 23.5 & 18.7 & 15.0 \\
\hline Bilateral & 437.0 & 471.3 & 477.1 & 577.7 & 560.3 \\
\hline \multicolumn{6}{|l|}{ Of which: } \\
\hline Russia 1/ & 81.0 & 75.1 & 78.3 & 136.3 & 133.3 \\
\hline United States & 85.0 & 83.4 & 81.1 & 77.7 & 73.9 \\
\hline Germany & 166.0 & 136.0 & 147.2 & 172.9 & 182.0 \\
\hline Japan & 32.0 & 28.6 & 27.2 & 34.2 & 30.8 \\
\hline Total short-term external debt (end-of period) 2/ & 1298.6 & 1169.1 & 1281.8 & 1639.3 & 1812.9 \\
\hline Trade credits & 622.3 & 530.3 & 475.5 & 531.7 & 661.7 \\
\hline Liabilities of the banking system & 112.4 & 114.1 & 188.1 & 276.9 & 279.0 \\
\hline Arrears & 462.1 & 433.5 & 488.5 & 433.9 & 353.5 \\
\hline \multicolumn{6}{|l|}{ Of which: } \\
\hline Gas & 234.3 & 138.6 & 224.2 & 214.0 & 147.4 \\
\hline Oil & 19.3 & 22.3 & 16.2 & 12.8 & 6.2 \\
\hline Electricity & 96.4 & 79.8 & 87.6 & 54.0 & 30.8 \\
\hline Other & 102.0 & 91.2 & 129.7 & 396.8 & 518.7 \\
\hline
\end{tabular}

Sources: Belarusian authorities; and Fund staff estimates.

1/ Fund staff estimate on an annualized basis.

2/ Short-term debt data are for Q3 2003. 
Table 44. Belarus: Disbursements and Payments on Medium- and Long-term Public and Publiclyguaranteed Debt, 1999-2003

(In millions of U.S. dollars; end of period)

\begin{tabular}{|c|c|c|c|c|c|}
\hline & 1999 & 2000 & 2001 & 2002 & 2003 \\
\hline \multicolumn{6}{|c|}{ Disbursements of medium- and long-term public and publicly- } \\
\hline guaranteed debt, excluding IMF & 39 & 153 & 118 & 143 & 50 \\
\hline Multilateral (Official) & 6 & 10 & 11 & 7 & 2 \\
\hline World Bank & 1 & 6 & 7 & 3 & 2 \\
\hline EBRD & 5 & 4 & 4 & 4 & 0 \\
\hline European Union & 0 & 0 & 0 & 0 & 0 \\
\hline Bilateral (Official) & 33 & 144 & 107 & 136 & 48 \\
\hline Russia & 3 & 0 & 30 & 70 & 0 \\
\hline Germany & 11 & 26 & 37 & 38 & 33 \\
\hline Other & 19 & 117 & 40 & 28 & 15 \\
\hline Other creditors & 0 & 0 & 0 & 0 & 0 \\
\hline \multicolumn{6}{|c|}{ Payments on existing stock of medium- and long-term public } \\
\hline and publicly-guaranteed debt, including IMF & 189 & 222 & 222 & 194 & 200 \\
\hline Principal & 140 & 181 & 171 & 156 & 168 \\
\hline Interest & 49 & 41 & 51 & 38 & 32 \\
\hline IMF & 69 & 60 & 35 & 32 & 33 \\
\hline Principal & 58 & 56 & 30 & 30 & 32 \\
\hline Interest & 11 & 4 & 5 & 2 & 1 \\
\hline World Bank & 21 & 22 & 19 & 19 & 19 \\
\hline Principal & 13 & 15 & 14 & 14 & 15 \\
\hline Interest & 8 & 7 & 5 & 5 & 4 \\
\hline EBRD & 25 & 37 & 26 & 21 & 17 \\
\hline Principal & 15 & 30 & 19 & 16 & 13 \\
\hline Interest & 10 & 7 & 7 & 5 & 4 \\
\hline Bilateral and EU & 74 & 103 & 142 & 122 & 131 \\
\hline Principal & 54 & 80 & 108 & 96 & 108 \\
\hline Interest & 20 & 23 & 34 & 26 & 23 \\
\hline
\end{tabular}

Source: Belarusian authorities. 
Table 45. Belarus: Gas Supply and Arrears, 1999-2003 (In millions of U.S. dollars; end-period stocks)

\begin{tabular}{|c|c|c|c|c|c|c|c|}
\hline & & \multirow{2}{*}{$\begin{array}{l}\text { Cost of gas } \\
\text { supplied }\end{array}$} & \multirow{2}{*}{$\begin{array}{l}\text { Total stock of } \\
\text { arrears for gas } \\
\text { supplied 1/ }\end{array}$} & \multicolumn{2}{|c|}{ Of which: } & \multirow{2}{*}{$\begin{array}{c}\text { Supply of } \\
\text { natural gas } \\
\text { (in millions of } \\
\text { m3) }\end{array}$} & \multirow{2}{*}{$\begin{array}{c}\text { Price } \\
\text { (\$ per thousand } \\
\text { m3) }\end{array}$} \\
\hline & & & & $\begin{array}{c}\text { Arrears to } \\
\text { Gazprom }\end{array}$ & $\begin{array}{l}\text { Arrears to other } \\
\text { creditors }\end{array}$ & & \\
\hline \multirow[t]{13}{*}{1999} & Jan & 52.2 & 355.9 & 216.4 & 139.5 & 1.738 & 30.00 \\
\hline & Feb & 44.4 & 336.0 & 245.2 & 90.8 & 1.479 & 30.00 \\
\hline & Mar & 41.6 & 318.1 & 280.7 & 37.4 & 1.385 & 30.00 \\
\hline & Apr & 37.8 & 302.5 & 273.1 & 29.4 & 1.261 & 30.00 \\
\hline & May & 34.8 & 298.6 & 273.1 & 25.5 & 1.161 & 30.00 \\
\hline & Jun & 31.3 & 310.0 & 286.6 & 23.4 & 1.029 & 30.46 \\
\hline & Jul & 32.1 & 307.9 & 272.5 & 35.4 & 1.049 & 30.51 \\
\hline & Aug & 33.0 & 305.7 & 261.7 & 44.0 & 1.082 & 30.52 \\
\hline & Sep & 34.5 & 317.2 & 259.0 & 58.2 & 1.135 & 30.41 \\
\hline & Oct & 41.7 & 320.5 & 279.9 & 40.6 & 1.373 & 30.42 \\
\hline & Nov & 49.0 & 266.1 & 218.1 & 48.0 & 1.634 & 30.00 \\
\hline & Dec & 53.6 & 274.1 & 198.0 & 76.1 & 1.750 & 30.42 \\
\hline & Total & 486.0 & & & & 16.076 & \\
\hline \multirow[t]{13}{*}{2000} & Jan & 54.7 & 308.7 & 220.8 & 87.9 & 1.795 & 30.44 \\
\hline & Feb & 50.5 & 316.7 & 238.6 & 78.1 & 1.657 & 30.46 \\
\hline & Mar & 52.9 & 326.4 & 236.6 & 89.8 & 1.734 & 30.48 \\
\hline & Apr & 40.0 & 334.6 & 239.4 & 95.2 & 1.312 & 30.49 \\
\hline & May & 34.0 & 328.2 & 224.1 & 104.1 & 1.111 & 30.60 \\
\hline & Jun & 32.7 & 312.6 & 225.0 & 87.6 & 1.068 & 30.60 \\
\hline & Jul & 33.0 & 315.6 & 227.9 & 87.7 & 1.079 & 30.58 \\
\hline & Aug & 34.1 & 282.7 & 209.6 & 73.1 & 1.114 & 30.62 \\
\hline & Sep & 34.0 & 273.9 & 212.3 & 61.6 & 1.111 & 30.62 \\
\hline & Oct & 43.0 & 261.9 & 210.3 & 51.6 & 1.404 & 30.55 \\
\hline & Nov & 43.9 & 305.9 & 251.6 & 54.3 & 1.438 & 30.53 \\
\hline & Dec & 50.1 & 209.1 & 136.9 & 72.2 & 1.641 & 30.51 \\
\hline & & 502.9 & & & & 16.464 & \\
\hline \multirow[t]{13}{*}{2001} & Jan & 53.7 & 202.1 & 128.9 & 73.2 & 1.761 & 30.51 \\
\hline & Feb & 48.5 & 183.4 & 146.4 & 37.0 & 1.590 & 30.52 \\
\hline & Mar & 54.8 & 179.0 & 174.0 & 5.0 & 1.796 & 30.51 \\
\hline & Apr & 40.3 & 174.6 & 162.1 & 12.5 & 1.319 & 30.54 \\
\hline & May & 33.1 & 184.1 & 161.2 & 22.9 & 1.080 & 30.62 \\
\hline & Jun & 32.0 & 195.1 & 175.5 & 19.6 & 1.045 & 30.60 \\
\hline & Jul & 33.4 & 191.0 & 175.9 & 15.1 & 1.089 & 30.69 \\
\hline & Aug & 33.6 & 191.1 & 173.6 & 17.5 & 1.096 & 30.62 \\
\hline & Sep & 34.4 & 194.5 & 181.7 & 12.8 & 1.098 & 30.63 \\
\hline & Oct & 40.7 & 217.5 & 192.4 & 25.1 & 1.334 & 30.54 \\
\hline & Nov & 46.9 & 226.9 & 200.1 & 26.8 & 1.538 & 30.54 \\
\hline & Dec & 53.9 & 239.2 & 202.7 & 36.5 & 1.765 & 30.54 \\
\hline & Total & 505.3 & & & & 16.511 & \\
\hline \multirow[t]{13}{*}{2002} & Jan & 54.0 & 252.4 & 200.1 & 52.3 & 1.768 & 30.52 \\
\hline & Feb & 46.0 & 250.5 & 216.2 & 34.3 & 1.510 & 30.51 \\
\hline & Mar & 50.3 & 252.9 & 244.4 & 8.5 & 1.652 & 30.45 \\
\hline & Apr & 42.5 & 271.3 & 258.2 & 13.1 & 1.393 & 30.45 \\
\hline & May & 22.5 & 263.4 & 259.4 & 4.0 & 1.048 & 21.49 \\
\hline & Jun & 21.6 & 255.3 & 243.5 & 11.8 & 0.944 & 22.89 \\
\hline & Jul & 25.1 & 248.7 & 247.0 & 1.7 & 1.030 & 24.41 \\
\hline & Aug & 23.8 & 249.8 & 230.6 & 19.2 & 0.991 & 24.38 \\
\hline & Sep & 31.3 & 255.6 & 240.6 & 15.0 & 1.088 & 28.53 \\
\hline & Oct & 36.7 & 269.9 & 247.1 & 22.8 & 1.509 & 24.30 \\
\hline & Nov & 23.0 & 243.9 & 193.7 & 50.2 & 0.781 & 29.50 \\
\hline & Dec & 49.9 & 208.7 & 129.4 & 79.3 & 1.281 & 38.91 \\
\hline & Total & 426.7 & & & & $14.995 \quad 2 /$ & \\
\hline \multirow[t]{13}{*}{2003} & Jan & 58.3 & 230.0 & 161.7 & 68.3 & 1.816 & 32.09 \\
\hline & Feb & 52.6 & 230.2 & 156.1 & 74.1 & 1.619 & 32.37 \\
\hline & Mar & 59.5 & 230.7 & 156.3 & 74.4 & 1.792 & 33.16 \\
\hline & Apr & 55.9 & 230.8 & 147.7 & 83.1 & 1.505 & 37.01 \\
\hline & May & 40.6 & 208.6 & 142.2 & 66.4 & 1.113 & 36.47 \\
\hline & Jun & 42.1 & 212.6 & 145.6 & 67.0 & 1.125 & 37.05 \\
\hline & Jul & 42.1 & 212.3 & 134.6 & 77.7 & 1.098 & 38.39 \\
\hline & Aug & 48.0 & 220.2 & 126.9 & 93.3 & 1.155 & 41.60 \\
\hline & Sep & 47.7 & 201.7 & 123.9 & 77.8 & 1.171 & 40.80 \\
\hline & Oct & 56.3 & 202.2 & 143.5 & 58.7 & 1.533 & 36.67 \\
\hline & Nov & 54.8 & 213.4 & 162.0 & 51.4 & 1.518 & 36.11 \\
\hline & Dec & 61.8 & 192.6 & 166.0 & 26.6 & 1.710 & 36.17 \\
\hline & Total & 619.7 & & & & $171552 /$ & \\
\hline
\end{tabular}

Source: Ministry of Statistics and Analysis; Beltransgaz

1/ Arrears include penalties on arrears accumulated.

2/ Not including gas supply under direct contracts: 2.582 millions of cub. m for 2002 and 0.958 millions of cub. $\mathrm{m}$ for 2003. 
Table 46. Belarus: Import and Consumption of Natural Gas, Monthly, 2000-03

\begin{tabular}{|c|c|c|c|c|c|c|c|c|}
\hline & & $\begin{array}{l}\text { Cost of imported gas } \\
\text { (thous. \$) }\end{array}$ & $\begin{array}{l}\text { Import of natural } \\
\text { gas } \\
\text { (in thousands of } \\
\mathrm{m} 3 \text { ) }\end{array}$ & $\begin{array}{c}\text { Price } \\
\text { (\$ per thousand } \\
\text { m3) }\end{array}$ & $\begin{array}{l}\text { Domestic } \\
\text { consumption (in } \\
\text { thousands of } \\
\text { m3) }\end{array}$ & $\begin{array}{c}\text { Domestic } \\
\text { production (in } \\
\text { thousands of } \\
\mathrm{m} 3 \text { ) }\end{array}$ & $\begin{array}{l}\text { Change in stock } \\
\text { (in thousands of } \\
\text { m3) }\end{array}$ & $\begin{array}{c}\text { Losses in } \\
\text { distribution (in } \\
\text { thousands of } \\
\mathrm{m} 3 \text { ) }\end{array}$ \\
\hline \multirow[t]{13}{*}{2000} & Jan & 56,226 & 1,845 & 30.5 & 1,934 & 21 & 68 & - \\
\hline & Feb & 52,216 & 1,707 & 30.6 & 1,781 & 20 & 54 & - \\
\hline & Mar & 54,244 & 1,774 & 30.6 & 1,814 & 22 & 18 & - \\
\hline & Apr & 41,728 & 1,362 & 30.6 & 1,339 & 21 & -44 & - \\
\hline & May & 35,717 & 1,161 & 30.8 & 1,161 & 23 & -23 & - \\
\hline & Jun & 34,513 & 1,120 & 30.8 & 1,118 & 23 & -25 & - \\
\hline & Jul & 34,824 & 1,130 & 30.8 & 1,061 & 23 & -92 & - \\
\hline & Aug & 35,843 & 1,164 & 30.8 & 1,105 & 21 & -80 & - \\
\hline & Sep & 36,048 & 1,173 & 30.7 & 1,165 & 21 & -29 & - \\
\hline & Oct & 45,767 & 1,478 & 31.0 & 1,464 & 21 & -35 & - \\
\hline & Nov & 46,627 & 1,514 & 30.8 & 1,614 & 20 & 80 & - \\
\hline & Dec & 51,785 & 1,687 & 30.7 & 1,777 & 21 & 69 & - \\
\hline & TOTAL & 525,538 & 17,115 & 30.7 & 17,333 & 257 & -39 & 229 \\
\hline \multirow[t]{13}{*}{2001} & Jan & 56,066 & 1,820 & 30.8 & 1,915 & 22 & 73 & - \\
\hline & Feb & 51,257 & 1,657 & 30.9 & 1,738 & 20 & 61 & - \\
\hline & Mar & 57,645 & 1,863 & 30.9 & 1,888 & 22 & 3 & - \\
\hline & Apr & 42,922 & 1,383 & 31.0 & 1,343 & 21 & -61 & - \\
\hline & May & 35,994 & 1,149 & 31.3 & 1,159 & 22 & -12 & - \\
\hline & Jun & 34,868 & 1,114 & 31.3 & 1,121 & 21 & -14 & - \\
\hline & Jul & 35,719 & 1,142 & 31.3 & 1,080 & 22 & -84 & - \\
\hline & Aug & 35,595 & 1,142 & 31.2 & 1,080 & 23 & -85 & - \\
\hline & Sep & 36,727 & 1,170 & 31.4 & 1,130 & 19 & -59 & - \\
\hline & Oct & 42,832 & 1,380 & 31.0 & 1,394 & 22 & -8 & - \\
\hline & Nov & 48,793 & 1,577 & 30.9 & 1,664 & 20 & 67 & - \\
\hline & Dec & 58,331 & 1,869 & 31.2 & 1,897 & 21 & 7 & - \\
\hline & TOTAL & 536,749 & 17,266 & 31.1 & 17,409 & 255 & -112 & 202 \\
\hline \multirow[t]{13}{*}{2002} & Jan & 58,078 & 1,865 & 31.1 & 1,951 & 22 & 64 & - \\
\hline & $\mathrm{Feb}$ & 49,460 & 1,592 & 31.1 & 1,652 & 20 & 40 & - \\
\hline & Mar & 53,821 & 1,739 & 31.0 & 1,779 & 22 & 18 & - \\
\hline & Apr & 45,364 & 1,463 & 31.0 & 1,489 & 21 & 5 & - \\
\hline & May & 38,901 & 1,280 & 30.4 & 1,250 & 21 & -51 & - \\
\hline & Jun & 29,288 & 1,145 & 25.6 & 1,055 & 21 & -111 & - \\
\hline & Jul & 32,106 & 1,183 & 27.1 & 1,072 & 22 & -133 & - \\
\hline & Aug & 35,412 & 1,245 & 28.4 & 1,140 & 21 & -126 & - \\
\hline & Sep & 33,440 & 1,250 & 26.7 & 1,163 & 18 & -105 & - \\
\hline & Oct & 42,145 & 1,630 & 25.9 & 1,616 & 20 & -34 & - \\
\hline & Nov & 46,678 & 1,339 & 34.8 & 1,517 & 19 & 159 & - \\
\hline & Dec & 73,857 & 1,847 & 40.0 & 2,038 & 17 & 174 & - \\
\hline & TOTAL & 538,549 & 17,578 & 30.6 & 17,722 & 244 & -100 & 195 \\
\hline \multirow[t]{13}{*}{2003} & Jan & 62,855 & 1,907 & 33.0 & 2,029 & 20 & 102 & - \\
\hline & $\mathrm{Feb}$ & 56,833 & 1,700 & 33.4 & 1,825 & 18 & 107 & - \\
\hline & Mar & 64,270 & 1,883 & 34.1 & 1,894 & 22 & -11 & - \\
\hline & Apr & 59,612 & 1,592 & 37.4 & 1,549 & 21 & -64 & - \\
\hline & May & 45,146 & 1,197 & 37.7 & 1,130 & 24 & -91 & - \\
\hline & Jun & 45,169 & 1,191 & 37.9 & 1,136 & 21 & -76 & - \\
\hline & Jul & 44,390 & 1,141 & 38.9 & 1,030 & 21 & -132 & - \\
\hline & Aug & 51,959 & 1,232 & 42.2 & 1,141 & 21 & -112 & - \\
\hline & Sep & 51,009 & 1,235 & 41.3 & 1,323 & 18 & 70 & - \\
\hline & Oct & 61,446 & 1,625 & 37.8 & 1,427 & 22 & -220 & - \\
\hline & Nov & 59,628 & 1,606 & 37.1 & 1,706 & 22 & 78 & - \\
\hline & Dec & 66,899 & 1,804 & 371.0 & 2,058 & 24 & 230 & - \\
\hline & TOTAL & 669,216 & 18,113 & 36.9 & 18,448 & 254 & 81 & 208 \\
\hline
\end{tabular}

Source: Ministry of Statistics and Analysis. 
Table 47. Belarus: Production, Trade and Consumption of Crude Oil, Monthly, 2000-03

\begin{tabular}{|c|c|c|c|c|c|c|c|}
\hline & \multicolumn{3}{|c|}{ Import } & \multirow{2}{*}{$\begin{array}{c}\begin{array}{c}\text { Domestic } \\
\text { production }\end{array} \\
\begin{array}{c}\text { Extraction } \\
\text { of oil }\end{array} \\
\text { (in thous.tons) }\end{array}$} & \multirow{2}{*}{$\begin{array}{c}\begin{array}{c}\text { Domestic } \\
\text { consumption }\end{array} \\
\begin{array}{c}\text { Consumption } \\
\text { of oil }\end{array} \\
\text { (in thous. tons) }\end{array}$} & \multirow{2}{*}{$\frac{\text { Export }}{\text { Export of oil }}$} & \multirow{2}{*}{$\begin{array}{c}\text { Stock } \\
\text { Change in stock } \\
\text { (in thousands of } \\
\text { tons) }\end{array}$} \\
\hline & $\begin{array}{l}\text { Cost of oil } \\
\text { imported } \\
\text { (thous. \$) }\end{array}$ & $\begin{array}{r}\text { Import of oil } \\
\text { (in thous. tons) }\end{array}$ & $\begin{array}{c}\text { Price } \\
\text { (\$ per ton) }\end{array}$ & & & & \\
\hline 2000 Jan & 124,708 & 989 & 126.1 & 155 & 1,081 & - & -63 \\
\hline Feb & 138,525 & 1,141 & 121.4 & 145 & 1,193 & 100 & 7 \\
\hline Mar & 107,786 & 914 & 118.0 & 157 & 1,112 & 50 & 91 \\
\hline Apr & 118,481 & 1,003 & 118.2 & 154 & 1,101 & 51 & -5 \\
\hline May & 115,262 & 970 & 118.8 & 159 & 1,143 & 0 & 14 \\
\hline Jun & 136,858 & 1,144 & 119.6 & 159 & 1,240 & - & -63 \\
\hline Jul & 131,825 & 1,055 & 124.9 & 157 & 1,276 & - & 64 \\
\hline Aug & 144,496 & 1,069 & 135.2 & 157 & 1,189 & 100 & 63 \\
\hline Sep & 120,801 & 883 & 136.8 & 152 & 943 & 50 & -42 \\
\hline Oct & 142,441 & 836 & 170.4 & 156 & 981 & - & -11 \\
\hline Nov & 170,087 & 952 & 178.7 & 148 & 1,045 & - & -55 \\
\hline Dec & 185,055 & 1,054 & 175.5 & 152 & 1,153 & - & -53 \\
\hline Year & $1,636,324$ & 12,010 & 136.2 & 1,851 & 13,457 & 351 & -53 \\
\hline 2001 Jan & 108,049 & 884 & 122.2 & 156 & 1,022 & 50 & 32 \\
\hline Feb & 115,818 & 998 & 116.0 & 144 & 1,084 & 50 & -8 \\
\hline Mar & 112,027 & 953 & 117.6 & 159 & 1,101 & 50 & 39 \\
\hline Apr & 118,895 & 999 & 118.9 & 155 & 1,100 & - & -54 \\
\hline May & 109,799 & 919 & 119.5 & 160 & 1,141 & - & 62 \\
\hline Jun & 140,266 & 1,157 & 121.2 & 155 & 1,228 & 50 & -34 \\
\hline Jul & 125,476 & 1,043 & 120.3 & 159 & 1,209 & 50 & 57 \\
\hline Aug & 103,358 & 897 & 115.3 & 161 & 1,050 & 51 & 43 \\
\hline Sep & 100,600 & 839 & 119.9 & 148 & 910 & 50 & -27 \\
\hline Oct & 120,533 & 991 & 121.6 & 156 & 1,016 & 49 & -82 \\
\hline Nov & 120,512 & 1,051 & 114.7 & 148 & 1,109 & 1 & -89 \\
\hline Dec & 104,305 & 1,182 & 88.3 & 151 & 1,345 & - & 12 \\
\hline Year & $1,379,638$ & 11,913 & 115.8 & 1,852 & 13,315 & 401 & -49 \\
\hline 2002 Jan & 93,391 & 1,237 & 75.5 & 156 & 1,404 & 50 & 61 \\
\hline Feb & 88,322 & 1,288 & 68.6 & 142 & 1,292 & 75 & -63 \\
\hline Mar & 83,679 & 1,318 & 63.5 & 158 & 1,437 & 75 & 36 \\
\hline Apr & 93,029 & 1,203 & 77.4 & 154 & 1,398 & 80 & 121 \\
\hline May & 113,442 & 1,181 & 96.0 & 160 & 1,203 & 80 & -58 \\
\hline Jun & 100,637 & 889 & 113.1 & 154 & 1,047 & 1 & 5 \\
\hline Jul & 123,477 & 1,028 & 120.1 & 159 & 1,128 & 60 & 1 \\
\hline Aug & 131,837 & 1,018 & 129.5 & 157 & 1,181 & - & 6 \\
\hline Sep & 174,644 & 1,263 & 138.3 & 150 & 1,367 & - & -46 \\
\hline Oct & 168,516 & 1,095 & 153.9 & 154 & 1,209 & 60 & 20 \\
\hline Nov & 162,923 & 1,158 & 140.7 & 147 & 1,222 & 60 & -23 \\
\hline Dec & 170,624 & 1,346 & 126.8 & 150 & 1,344 & 60 & -92 \\
\hline Year & $1,504,521$ & 14,024 & 107.3 & 1,841 & 15,232 & 601 & -32 \\
\hline 2003 Jan & 190,913 & 1,399 & 136.5 & 151 & 1,391 & 70 & -89 \\
\hline Feb & 180,197 & 1,261 & 142.9 & 136 & 1,329 & 68 & 0 \\
\hline Mar & 177,921 & 1,186 & 150.1 & 153 & 1,460 & 72 & 193 \\
\hline Apr & 146,529 & 1,320 & 111.0 & 149 & 1,378 & 70 & -21 \\
\hline May & 139,194 & 1,434 & 97.1 & 157 & 1,373 & 70 & -148 \\
\hline Jun & 148,818 & 1,303 & 114.2 & 154 & 1,502 & 70 & 115 \\
\hline Jul & 164,081 & 1,289 & 127.2 & 160 & 1,442 & 70 & 63 \\
\hline Aug & 166,725 & 1,224 & 136.2 & 159 & 1,336 & 70 & 23 \\
\hline Sep & 140,269 & 903 & 155.3 & 149 & 1,078 & 70 & 96 \\
\hline Oct & 154,916 & 1,064 & 145.7 & 156 & 855 & 95 & -270 \\
\hline Nov & 174,136 & 1,189 & 146.5 & 148 & 1,343 & 27 & 33 \\
\hline Dec & 199,333 & 1,313 & 151.8 & 148 & 1,284 & 48 & -129 \\
\hline Year & $1,983,032$ & 14,885 & 133.2 & 1,820 & 15,770 & 801 & -134 \\
\hline
\end{tabular}


Table 48. Belarus: Production, Trade and Consumption of Electricity, Monthly, 2000-03

\begin{tabular}{|c|c|c|c|c|c|c|c|c|c|c|}
\hline & & \multicolumn{3}{|c|}{ Import } & \multirow{2}{*}{$\begin{array}{c}\begin{array}{c}\text { Domestic } \\
\text { production }\end{array} \\
\text { Production of } \\
\text { electricity } \\
\text { (mln. kWh) }\end{array}$} & \multirow{2}{*}{$\begin{array}{c}\begin{array}{c}\text { Domestic } \\
\text { consumption }\end{array} \\
\begin{array}{c}\text { Consumption } \\
\text { of electricity } \\
(\mathrm{mln} . \mathrm{kWh})\end{array}\end{array}$} & \multicolumn{3}{|c|}{ Export } & \multirow{2}{*}{$\begin{array}{c}\text { Losses } \\
\text { Losses in } \\
\text { distribution (mln } \\
\mathrm{kWh})\end{array}$} \\
\hline & & $\begin{array}{l}\text { Cost of electricity } \\
\text { imported } \\
\text { (thous. \$) }\end{array}$ & $\begin{array}{c}\text { Import of oil } \\
\text { (mln. kWh) }\end{array}$ & $\begin{array}{c}\text { Price } \\
(\$ \text { per kWh })\end{array}$ & & & $\begin{array}{l}\text { Value of electricity } \\
\text { exported (thous. \$) }\end{array}$ & $\begin{array}{c}\text { Export of } \\
\text { electricity (mln. } \\
\mathrm{kWh})\end{array}$ & $\begin{array}{c}\text { Price } \\
(\$ \text { per kWh })\end{array}$ & \\
\hline \multirow[t]{13}{*}{2000} & Jan & 10,919 & 606 & 18.0 & 2,689 & 3,294 & 18 & 1 & 18.3 & - \\
\hline & Feb & 10,334 & 574 & 18.0 & 2,498 & 3,071 & 16 & 1 & 18.5 & - \\
\hline & Mar & 10,621 & 590 & 18.0 & 2,563 & 3,152 & 14 & 1 & 17.7 & - \\
\hline & Apr & 9,352 & 502 & 18.6 & 2,136 & 2,637 & 15 & 1 & 19.2 & - \\
\hline & May & 7,025 & 397 & 17.7 & 2,000 & 2,396 & 12 & 1 & 17.1 & - \\
\hline & Jun & 7,097 & 406 & 17.5 & 1,895 & 2,301 & 10 & 0 & 18.0 & - \\
\hline & Jul & 10,273 & 571 & 18.0 & 1,798 & 2,369 & 12 & 0 & 17.8 & - \\
\hline & Aug & 13,590 & 755 & 18.0 & 1,784 & 2,539 & - & - & - & - \\
\hline & Sep & 14,834 & 824 & 18.0 & 1,708 & 2,531 & 14 & 1 & 18.5 & - \\
\hline & Oct & 10,400 & 589 & 17.7 & 2,209 & 2,797 & 17 & 1 & 17.4 & - \\
\hline & Nov & 10,290 & 604 & 17.0 & 2,422 & 3,025 & 15 & 1 & 17.4 & - \\
\hline & Dec & 14,427 & 802 & 18.0 & 2,399 & 3,200 & 18 & 1 & 17.6 & - \\
\hline & Year & 129,162 & 7,220 & 17.9 & 26,101 & 33,312 & 161 & 9 & 17.9 & 3,410 \\
\hline \multirow[t]{13}{*}{2001} & Jan & 12,014 & 709 & 16.9 & 2,500 & 3,209 & - & - & - & - \\
\hline & Feb & 13,063 & 773 & 16.9 & 2,187 & 2,960 & - & - & - & - \\
\hline & Mar & 11,247 & 625 & 18.0 & 2,474 & 3,099 & - & - & - & - \\
\hline & Apr & 9,819 & 565 & 17.4 & 2,044 & 2,609 & - & - & - & - \\
\hline & May & 8,757 & 504 & 17.4 & 1,926 & 2,430 & - & - & - & - \\
\hline & Jun & 7,971 & 457 & 17.4 & 1,899 & 2,356 & 3 & 0 & 19.8 & - \\
\hline & $\mathrm{Jul}$ & 8,430 & 492 & 17.1 & 1,902 & 2,394 & - & - & - & - \\
\hline & Aug & 9,774 & 566 & 17.3 & 1,939 & 2,505 & 0 & 0 & 8.2 & - \\
\hline & Sep & 13,365 & 737 & 18.1 & 1,778 & 2,515 & - & - & - & - \\
\hline & Oct & 18,280 & 890 & 20.5 & 1,943 & 2,833 & 4 & 0 & 20.5 & - \\
\hline & Nov & 16,782 & 994 & 16.9 & 2,058 & 3,052 & 4 & 0 & 16.9 & - \\
\hline & Dec & 17,696 & 1,009 & 17.5 & 2,413 & 3,372 & 908 & 50 & 18.2 & - \\
\hline & Year & 147,199 & 8,321 & 17.7 & 25,063 & 33,334 & 920 & 50 & 18.2 & 3,455 \\
\hline \multirow[t]{13}{*}{2002} & Jan & 16,132 & 862 & 18.7 & 2,444 & 3,235 & 1,303 & 71 & 18.3 & - \\
\hline & Feb & 14,223 & 732 & 19.4 & 2,163 & 2,832 & 1,141 & 63 & 18.3 & - \\
\hline & Mar & 11,133 & 595 & 18.7 & 2,398 & 2,926 & 1,229 & 67 & 18.3 & - \\
\hline & Apr & 988 & 83 & 11.9 & 2,559 & 2,609 & 600 & 33 & 18.2 & - \\
\hline & May & 519 & 32 & 16.3 & 2,391 & 2,423 & - & - & - & - \\
\hline & Jun & 10,780 & 514 & 20.9 & 1,802 & 2,316 & - & - & - & - \\
\hline & Jul & 11,328 & 467 & 24.3 & 1,946 & 2,413 & 2 & 0 & 29.0 & - \\
\hline & Aug & 11,332 & 464 & 24.4 & 1,989 & 2,453 & 0 & 0 & 25.2 & - \\
\hline & Sep & 12,416 & 508 & 24.4 & 1,998 & 2,506 & - & - & - & - \\
\hline & Oct & 14,617 & 641 & 22.8 & 2,339 & 2,980 & - & - & - & - \\
\hline & Nov & 17,183 & 948 & 18.1 & 2,005 & 2,953 & 1 & 0 & 22.4 & - \\
\hline & Dec & 19,860 & 943 & 21.0 & 2,406 & 3,349 & 4 & 0 & 24.7 & - \\
\hline & Year & 140,511 & 6,789 & 20.7 & 26,440 & 32,995 & 4,281 & 234 & 18.3 & $\ldots$ \\
\hline \multirow[t]{13}{*}{2003} & Jan & 18,720 & 799 & 23.4 & 2,477 & 3,231 & 828 & 45 & 18.5 & - \\
\hline & Feb & 18,121 & 784 & 23.1 & 2,251 & 2,969 & 1,229 & 66 & 18.5 & - \\
\hline & Mar & 12,770 & 770 & 16.6 & 2,338 & 3,037 & 1,313 & 71 & 18.5 & - \\
\hline & Apr & 8,997 & 400 & 22.5 & 2,419 & 2,752 & 1,244 & 67 & 18.5 & - \\
\hline & May & 12,265 & 510 & 24.1 & 1,990 & 2,430 & 1,294 & 70 & 18.5 & - \\
\hline & Jun & 5,652 & 304 & 18.6 & 2,144 & 2,380 & 1,257 & 68 & 18.5 & - \\
\hline & Jul & 10,899 & 569 & 19.2 & 1,896 & 2,435 & 547 & 30 & 18.5 & - \\
\hline & Aug & 10,779 & 552 & 19.5 & 2,021 & 2,524 & 907 & 49 & 18.5 & - \\
\hline & Sep & 11,243 & 573 & 19.6 & 1,999 & 2,503 & 1,273 & 69 & 18.5 & - \\
\hline & Oct & 12,186 & 639 & 19.1 & 2,372 & 2,937 & 1,380 & 74 & 18.5 & - \\
\hline & Nov & 11,919 & 828 & 14.4 & 2,254 & 3,013 & 1,275 & 69 & 18.5 & - \\
\hline & Dec & 9,352 & 630 & 14.8 & 2,454 & 3,017 & 1,246 & 67 & 18.6 & - \\
\hline & Year & 142,903 & 7,359 & 19.4 & 26,615 & 33,228 & 13,793 & 746 & 18.5 & 3450 \\
\hline
\end{tabular}

Source: Ministry of Statistics and Analysis. 
Table 49. Belarus: Exports and Imports of Oil Products, Monthly, 2000-03

\begin{tabular}{|c|c|c|c|c|c|c|c|}
\hline & & \multicolumn{3}{|c|}{ Import } & \multicolumn{3}{|c|}{ Export } \\
\hline & & $\begin{array}{c}\text { Cost of oil } \\
\text { products imported } \\
\text { (thous. \$) }\end{array}$ & $\begin{array}{l}\text { Import of oil } \\
\text { products } \\
\text { (tons) }\end{array}$ & $\begin{array}{c}\text { Price } \\
(\$ \text { per ton })\end{array}$ & $\begin{array}{l}\text { Value of oil } \\
\text { products } \\
\text { exported } \\
\text { (thous. \$) }\end{array}$ & $\begin{array}{l}\text { Export of oil } \\
\text { products } \\
\text { (tons) }\end{array}$ & $\begin{array}{c}\text { Price } \\
\text { (\$ per ton) }\end{array}$ \\
\hline \multirow{13}{*}{2000} & Jan & 21,750 & 120,160 & 181.0 & 85,320 & 536,654 & 159.0 \\
\hline & Feb & 20,262 & 114,472 & 177.0 & 120,340 & 794,557 & 151.5 \\
\hline & Mar & 23,538 & 122,300 & 192.5 & 111,237 & 674,521 & 164.9 \\
\hline & Apr & 18,691 & 94,569 & 197.6 & 102,943 & 678,926 & 151.6 \\
\hline & May & 12,674 & 84,172 & 150.6 & 107,705 & 710,235 & 151.6 \\
\hline & Jun & 15,887 & 84,297 & 188.5 & 123,295 & 752,912 & 163.8 \\
\hline & Jul & 13,736 & 80,023 & 171.6 & 148,002 & 846,165 & 174.9 \\
\hline & Aug & 8,822 & 43,577 & 202.4 & 125,866 & 731,415 & 172.1 \\
\hline & Sep & 13,001 & 79,610 & 163.3 & 100,870 & 493,896 & 204.2 \\
\hline & Oct & 18,495 & 91,221 & 202.7 & 94,714 & 434,646 & 217.9 \\
\hline & Nov & 19,841 & 77,181 & 257.1 & 105,751 & 470,729 & 224.7 \\
\hline & Dec & 23,461 & 83,778 & 280.0 & 132,804 & 658,409 & 201.7 \\
\hline & Year & 210,158 & $1,075,360$ & 195.4 & $1,358,848$ & $7,783,065$ & 174.6 \\
\hline \multirow[t]{13}{*}{2001} & Jan & 12,412 & 40,837 & 303.9 & 92,375 & 520,245 & 177.6 \\
\hline & $\mathrm{Feb}$ & 9,466 & 32,505 & 291.2 & 130,979 & 818,762 & 160.0 \\
\hline & Mar & 11,545 & 53,146 & 217.2 & 83,817 & 547,377 & 153.1 \\
\hline & Apr & 10,159 & 38,825 & 261.7 & 115,695 & 731,046 & 158.3 \\
\hline & May & 6,250 & 26,724 & 233.9 & 112,096 & 707,137 & 158.5 \\
\hline & Jun & 7,210 & 29,629 & 243.3 & 110,092 & 668,800 & 164.6 \\
\hline & Jul & 4,443 & 21,757 & 204.2 & 127,511 & 783,818 & 162.7 \\
\hline & Aug & 4,954 & 21,371 & 231.8 & 102,872 & 635,520 & 161.9 \\
\hline & Sep & 6,455 & 34,612 & 186.5 & 104,081 & 605,745 & 171.8 \\
\hline & Oct & 6,503 & 28,408 & 228.9 & 67,820 & 382,974 & 177.1 \\
\hline & Nov & 7,316 & 31,381 & 233.1 & 63,645 & 471,099 & 135.1 \\
\hline & Dec & 4,511 & 17,005 & 265.3 & 97,677 & 785,061 & 124.4 \\
\hline & Year & 91,222 & 376,200 & 242.5 & $1,208,661$ & $7,657,584$ & 157.8 \\
\hline \multirow{13}{*}{2002} & Jan & 5,108 & 25,188 & 202.8 & 96,668 & 810,886 & 119.2 \\
\hline & Feb & 5,059 & 30,086 & 168.1 & 97,179 & 836,016 & 116.2 \\
\hline & Mar & 5,826 & 27,904 & 208.8 & 127,093 & $1,069,459$ & 118.8 \\
\hline & Apr & 5,278 & 22,214 & 237.6 & 125,911 & 977,301 & 128.8 \\
\hline & May & 4,331 & 10,644 & 406.9 & 129,393 & 894,707 & 144.6 \\
\hline & Jun & 2,948 & 8,001 & 368.4 & 134,454 & 840,195 & 160.0 \\
\hline & Jul & 4,692 & 13,933 & 336.7 & 88,249 & 577,609 & 152.8 \\
\hline & Aug & 8,013 & 38,812 & 206.5 & 126,107 & 751,381 & 167.8 \\
\hline & Sep & 10,212 & 57,847 & 176.5 & 129,666 & 760,579 & 170.5 \\
\hline & Oct & 13,512 & 75,022 & 180.1 & 148,877 & 840,203 & 177.2 \\
\hline & Nov & 16,628 & 92,916 & 179.0 & 116,916 & 640,340 & 182.6 \\
\hline & Dec & 15,702 & 88,255 & 177.9 & 158,274 & 876,340 & 180.6 \\
\hline & Year & 97,307 & 490,822 & 198.3 & $1,478,784$ & $9,875,016$ & 149.8 \\
\hline \multirow[t]{13}{*}{2003} & Jan & 16,203 & 127,006 & 127.6 & 178,827 & $1,026,130$ & 174.3 \\
\hline & Feb & 8,320 & 56,771 & 146.6 & 176,029 & 929,110 & 189.5 \\
\hline & Mar & 11,424 & 68,653 & 166.4 & 197,185 & 946,735 & 208.3 \\
\hline & Apr & 16,063 & 109,872 & 146.2 & 152,811 & 868,043 & 176.0 \\
\hline & May & 9,926 & 69,612 & 142.6 & 174,821 & $1,076,590$ & 162.4 \\
\hline & Jun & 8,755 & 46,606 & 187.9 & 168,701 & 980,174 & 172.1 \\
\hline & Jul & 8,470 & 37,770 & 224.3 & 162,721 & 899,583 & 180.9 \\
\hline & Aug & 6,967 & 24,001 & 290.3 & 156,092 & 813,814 & 191.8 \\
\hline & Sep & 14,594 & 75,889 & 192.3 & 87,122 & 420,949 & 207.0 \\
\hline & Oct & 20,113 & 123,639 & 162.7 & 101,118 & 522,151 & 193.7 \\
\hline & Nov & 20,548 & 130,577 & 157.4 & 176,973 & 929,669 & 190.4 \\
\hline & Dec & 21,006 & 133,906 & 156.9 & 222,164 & $1,126,471$ & 197.2 \\
\hline & Year & 162,441 & $1,004,618$ & 161.7 & $1,954,564$ & $10,539,419$ & 185.5 \\
\hline
\end{tabular}

Source: Ministry of Statistics and Analysis. 
Table 50. Belarus: Transformation of Property, 1999-2003

\begin{tabular}{|c|c|c|c|c|c|}
\hline & 1999 & 2000 & 2001 & 2002 & 2003 \\
\hline & \multicolumn{4}{|c|}{ (Number of enterprises) } & \\
\hline \multicolumn{6}{|l|}{ Republican level } \\
\hline Number of transformed enterprises & 106 & 52 & 24 & 89 & 64 \\
\hline Cumulative since 1991 & 780 & 832 & 856 & 945 & 1,009 \\
\hline \multicolumn{6}{|l|}{ Transformed by activity } \\
\hline Industry & 21 & 16 & 11 & 45 & 14 \\
\hline Construction & 45 & 18 & 6 & 12 & 10 \\
\hline Communication and transport & 2 & 1 & 1 & 0 & 5 \\
\hline Other sectors & 38 & 17 & 6 & 32 & 35 \\
\hline \multicolumn{6}{|l|}{ Transformed by method } \\
\hline Conversion into joint-stock companies & 90 & 49 & 24 & 89 & 64 \\
\hline Buy-outs of leased companies & 16 & 0 & 0 & 0 & 0 \\
\hline Sale by tender & 0 & 2 & 0 & 0 & 0 \\
\hline Sale in auctions & 0 & 1 & 0 & 0 & 0 \\
\hline \multicolumn{6}{|l|}{ Communal level } \\
\hline Number of transformed enterprises & 201 & 125 & 70 & 105 & 162 \\
\hline Cumulative since 1991 & 2,422 & 2,547 & 2,617 & 2,722 & 2,884 \\
\hline \multicolumn{6}{|l|}{ Transformed by activity } \\
\hline Trade & 85 & 37 & 30 & 48 & 47 \\
\hline Restaurants & 17 & 4 & 8 & 3 & 2 \\
\hline Housing services & 31 & 27 & 7 & 21 & 19 \\
\hline Industry & 29 & 3 & 9 & 4 & 22 \\
\hline Other sectors & 39 & 54 & 16 & 29 & 72 \\
\hline \multicolumn{6}{|l|}{ Transformed by method } \\
\hline Conversion into joint-stock companies & 94 & 81 & 39 & 68 & 147 \\
\hline Buy-outs of leased companies & 33 & 13 & 5 & 3 & 1 \\
\hline Sale by tender & 14 & 16 & 12 & 17 & 3 \\
\hline Sale in auctions & 60 & 15 & 12 & 17 & 11 \\
\hline Other employee buy-outs & 0 & 0 & 2 & 0 & 0 \\
\hline
\end{tabular}

Source: Ministry of Economy. 